\title{
Stability Theory of Difference Approximations for Multidimensional Initial-Boundary Value Problems
}

\author{
By Daniel Michelson
}

\begin{abstract}
A stability theory is developed for dissipative difference approximations to multidimensional initial-boundary value problems. The original differential problem should be strictly hyperbolic and the difference problem consistent with the differential one. An algebra of pseudo-difference operators is built and later used to prove the stability of the difference approximation with variable coefficients. In addition, stability of the Cauchy problem for weakly dissipative difference approximations with variable coefficients is proved.
\end{abstract}

0. Introduction. In the last two decades the mixed initial-boundary value problems for hyperbolic systems of partial differential equations were extensively and thoroughly studied. We should especially mention the classical work of Kreiss in [7] where he proved the basic a priori estimate for the strictly hyperbolic systems in the case of zero initial data, by constructing a pseudodifferential symmetrizer in the plane tangent to the boundary. This result was further generalized by Agranovich in [1] for a wider class of hyperbolic systems and extended by Rauch in [16] and Sarason in [17] to the case of nonzero initial data. Later Majda and Osher in [11] considered also characteristic boundaries.

On the other hand an intelligent numerical solution of these problems requires a stability analysis of their difference approximation based on a suitable stability theory. Such a theory was, however, developed only in the one space dimensional case, first by Kreiss in [8] and Osher in [14] for some two-step dissipative schemes and nonzero initial conditions and then by Gustafsson, Kreiss and Sundström in [5] for more general dissipative as well as strictly nondissipative schemes with zero initial conditions. An attempt to generalize these results for multidimensional problems encounters two main difficulties. One is that the Fourier symbol of the difference operator, though consistent with the original differential equations, may approximate a very complicated hyperbolic-parabolic system when the dual variables of the symbol are not in a neighborhood of zero. Although such situations cannot be investigated in general, some difference schemes such as Lax-Friedrichs, modified Lax-Wendroff and Burstein schemes, for which the symbol is a polynomial of one matrix, could be analyzed completely. Analysis of that type is carried out in [13] for the Burstein scheme and characteristic boundary. The second difficulty occurs in the construction of the Kreiss symmetrizer for the difference problem when

Received October 7, 1981

1980 Mathematics Subject Classification. Primary 65N10, 65M10.

Key words and phrases. Stability theory, initial-boundary value problems, difference approximations, hyperbolic systems.

* Research sponsored in part by the National Science Foundation Grant MCS 78-01252. 
the dual Fourier variables are near zero. One of the conditions used in [7] for the original construction required the corresponding Cauchy problem with the reverse time to be well posed. This is certainly not true for dissipative difference approximations. In Section 3 we show instead that Kreiss' construction applies directly to the dissipative case, so that one does not need the reversibility of the time in the Cauchy problem.

In this paper we consider only fully dissipative schemes, thus avoiding the described-above first difficulty. The boundary is noncharacteristic and the initial condition is zero. We believe that arguments similar to those in [17] could apply to the case of nonzero initial condition. One should, however, note that even for nonzero but sufficiently smooth initial data and smooth nonhomogeneity in the differential equations and boundary conditions, the solution of the difference problem converges to the solution of the differential one (e.g. see [6]).

We summarize now the contents of this paper. In Section 1 the definition of stability is given and the main results are stated. In Section 2 the difference problem is linearized and the stability estimate reformulated. In Section 3 the frozen coefficient problem is considered and the symmetrizer constructed. In Section 4 an algebra of pseudo-difference operators is introduced. In Section 5 the basic a priori estimate for the variable coefficients case is proven. In Section 6 the stability of the Cauchy problem for the weakly dissipative schemes and solvability of the initialboundary value problem are proven.

1. Definitions, Statements of Results. We consider a first order hyperbolic system of partial differential equations

$$
\frac{\partial u(x, t)}{\partial t}+\sum_{j=0}^{n-1} A_{j}(x, t) \frac{\partial u(x, t)}{\partial x_{j}}+C(x, t) u(x, t)=F(x, t) .
$$

Here $u(x, t)=\left(u^{(1)}(x, t), \ldots, u^{(k)}(x, t)\right)^{\prime}$ is a complex-valued vector function of the real variables $(x, t)$ with $x=\left(x_{0}, x_{1}, \ldots, x_{n-1}\right)=\left(x_{0}, x_{-}\right)$, and $A_{j}, C$ are $k \times k$ matrix-valued functions depending smoothly on $(x, t)$ which tend rapidly (i.e. faster than any power of $\left.(|x|+|t|)^{-1}\right)$ to the constant matrices $A_{j}(\infty)$ and $C(\infty)$ as $|x|+|t|$ tends to infinity. We assume that Eq. (1.1) is strictly hyperbolic, i.e. for all real $\omega=\left(\omega_{0}, \omega_{1}, \ldots, \omega_{n-1}\right)=\left(\omega_{0}, \omega_{-}\right)$with $|\omega| \neq 0$ the eigenvalues of the matrix $\sum_{j=0}^{n-1} A_{j}(x, t) \omega_{j}$ are real and distinct. Denote by $R^{+}$the set of nonnegative real numbers. The system in (1.1) is solved in the domain $(x, t) \in R^{+} \times R^{n-1} \times R^{+}$with the boundary condition

$$
S\left(x_{-}, t\right) u\left(0, x_{-}, t\right)=g\left(x_{-}, t\right), \quad\left(x_{-}, t\right) \in R^{n-1} \times R^{+}
$$

and initial condition

$$
u(x, 0)=f(x) \text { for } x \in R^{+} \times R^{n-1} .
$$

We assume that the boundary is noncharacteristic, i.e. $A_{0}(x, t)$ is nonsingular for $x_{0}=0$ and $S\left(x_{-}, t\right)$ is an $[l \times k]$ smooth matrix-valued function, where $l$ is the number of positive eigenvalues of $A_{0}\left(0, x_{-}, t\right)$, and $S\left(x_{-}, t\right)$ tends rapidly to a constant matrix as $\left|x_{-}\right|+|t| \rightarrow \infty$. For $f=0$ the well-posedness of the problem in 
(1.1)-(1.3) follows from the a priori estimate

$$
\begin{aligned}
\eta\left\|u(x, t) e^{-\eta t}\right\|^{2}+\left\|u\left(0, x_{-}, t\right) e^{-\eta t}\right\|^{2} & \\
& \leqslant K\left(\frac{1}{\eta}\left\|F(x, t) e^{-\eta t}\right\|^{2}+\left\|g\left(x_{-}, t\right) e^{-\eta t}\right\|^{2}\right)
\end{aligned}
$$

which should hold for any $\eta>\eta_{0} \geqslant 0$ and some constant $K$ independently of the function $u(x, t)$. Here $\|\cdot\|$ denotes the $L_{2}$ norm over domains indicated by the arguments. The problem in (1.1)-(1.3) was investigated by Kreiss in [7], where he showed that estimate (1.4) follows from the so-called uniform Kreiss condition (hereafter referred to as UKC) and in the case of constant coefficients is equivalent to this condition.

In order to define a difference approximation to the problem in (1.1)-(1.3) we introduce uniform grids $R_{h}^{n}$ in $R^{n}$ and $R_{h}^{+}$in $R^{+}$with the mesh size $h$ and consider grid vector functions $u(x, t)$ defined for $(x, t) \in R_{h}^{+} \times R_{h}^{n}$. We use the notation $E_{j}$, $j=0,1, \ldots, n-1$, for the displacement operator in $x_{j}$ direction

$$
\left(E_{j} u\right)(x, t)=u\left(x_{0}, \ldots, x_{j}+h, \ldots, t\right),
$$

and $E_{n}$ for the one in $t$ direction. The differential operator in (1.1) is approximated by a multilevel difference operator

$$
L\left(h, x, t, E_{x}, E_{n}\right)=\sum_{\sigma=0}^{-\sigma_{0}} \sum_{\nu=0}^{\nu_{0}} \sum_{\mu} L_{\nu, \mu, \sigma}(h, x, t) E_{0}^{\nu} E_{-}^{\mu} E_{n}^{\sigma},
$$

where

$$
E_{x}=\left(E_{0}, E_{1}, \ldots, E_{n-1}\right)=\left(E_{0}, E_{-}\right), \quad E_{-}^{\mu}=\prod_{j=1}^{n-1} E_{j}^{\mu_{j}},
$$

$L_{\nu, \mu, \sigma}(h, x, t)$ are matrix-valued functions of the order $k \times k$ depending smoothly on $(h, x, t) \in\left[0, h_{0}\right] \times R^{+} \times R^{n}$, the integers $\sigma_{0}, \nu_{0}$ are positive and the integer multiindex $\mu$ varies over a finite set. We also assume that the functions $L_{\nu, \mu, \sigma}$ are of the form

$$
L_{\nu, \mu, \sigma}(h, x, t)=L_{\nu, \mu, \sigma}(0, x, t)+h L_{\nu, \mu, \sigma}^{\prime}(h, x, t),
$$

where $L_{\nu, \mu, \sigma}^{\prime}(h, x, t)$ are uniformly bounded and $L_{\nu, \mu, \sigma}(0, x, t)$ tend rapidly (i.e. faster than any power of $\left.(|x|+|t|)^{-1}\right)$ to the constant matrices $L_{\nu, \mu, \sigma}(0, \infty)$ when $|x|+|t| \rightarrow \infty$. The boundary operator in (1.2) is replaced by a difference operator

$$
S\left(h, x_{-}, t, E_{x}, E_{n}\right)=\sum_{\sigma=0}^{-\sigma_{0}} \sum_{\nu=0}^{\nu_{0}-1} \sum_{\mu} S_{\nu, \mu, \sigma}\left(h, x_{-}, t\right) E_{0}^{\nu} E_{-}^{\mu} E_{n}^{\boldsymbol{\sigma}},
$$

where $S_{\nu, \mu, \sigma}$ satisfy the same conditions as $L_{\nu, \mu, \sigma}$ in (1.6). Note that we permit the matrices $L_{\nu_{0}, \mu, \sigma}$ and $S_{\nu_{0}-1, \mu, \sigma}$ to be zero. Finally, the differential problem in (1.1)-(1.3) is approximated by the difference problem
(A) $\quad L u(x, t)=h F(x, t), \quad(x, t) \in R_{h}^{+} \times R_{h}^{n-1} \times R_{h}^{+}, t \geqslant \sigma_{0} h$,
(B) $\quad S u\left(0, x_{-}, t\right)=g\left(x_{-}, t\right), \quad\left(x_{-}, t\right) \in R_{h}^{n-1} \times R_{h}^{+}, t \geqslant \sigma_{0} h$,
(C) $u(x, \sigma h)=f_{\sigma}(x), \quad x \in R_{h}^{+} \times R_{h}^{n-1}, 0 \leqslant \sigma \leqslant \sigma_{0}-1$. 
To give the definition of stability for the problem in (1.8) we introduce the scalar product

$$
\langle u, v\rangle_{x, t}=\sum_{(x, t) \in R_{h}^{+} \times R_{h}^{n}}(u(x, t), v(x, t)) \cdot h^{n+1}
$$

and the $l_{2}$ norm

$$
\|u\|_{x, t}^{2}=\langle u, u\rangle_{x, t} .
$$

If the function $u(x, t)$ is defined only for $t \geqslant 0$, we extend it to be zero for $t<0$. Similarly the scalar products \langle\rangle$_{x_{-}, t},\langle\rangle_{x},\langle\rangle_{x_{-}}$, and the corresponding norms are defined. We denote by $l_{2}(x, t)$ the space of grid functions $u(x, t)$ defined for $(x, t) \in R_{h}^{+} \times R_{h}^{n}$ with the scalar product in (1.9) and by $l_{2}(x), l_{2}\left(x_{-}\right)$the corresponding Hilbert spaces of the grid functions $u(x)$ and $u\left(x_{-}\right)$, respectively. In addition we introduce weighted norms

$$
\|u\|_{x, t, \eta}=\left\|e^{-\eta t} u\right\|_{x, t}, \quad \eta \geqslant 0,
$$

with the corresponding Hilbert spaces $e^{\eta t} l_{2}(x, t)$ and use the natural definitions for the norms \|\|$_{x, \eta}$ and \|\|$_{x_{-}, \eta}$. Our definition of stability is the same as Definition 3.3 in [5].

Definition 1.1. The problem in (1.8) with $f_{\sigma}=0$ is called stable if there exist $h_{0}>0$ and $\eta_{0} \geqslant 0$ such that for any $\eta>\eta_{0}$ and $h \leqslant h_{0}$ and any grid functions $F \in$ $e^{\eta t} l_{2}(x, t), g \in e^{\eta t} l_{2}\left(x_{-}, t\right)$ there exists a unique solution $u \in e^{\eta t} l_{2}(x, t)$ which satisfies the estimate

$$
\begin{aligned}
\frac{\eta}{1+\eta h}\|u(x, t)\|_{x, t, \eta}^{2} & +\sum_{\nu=0}^{\nu_{0}-1}\left\|u\left(\nu h, x_{-}, t\right)\right\|_{x_{-}, t, \eta}^{2} \\
& \leqslant K\left(\frac{1+\eta h}{\eta}\|F(x, t)\|_{x, t, \eta}^{2}+\left\|g\left(x_{-}, t\right)\right\|_{x_{-}, t, \eta}^{2}\right)
\end{aligned}
$$

As shown by Gustafsson in [6], such stability implies the convergence of the solution $u(x, t)$ in (1.8) to the solution of (1.1)-(1.3) even in the case $f \neq 0$ provided the problem in (1.8) is consistent with the one in (1.1)-(1.3) and the functions $F, g, f$ in (1.1)-(1.3) belong to the Sobolev spaces of sufficiently high order.

In order to solve problem (1.8) in time one should consider the operators

$$
L^{(0)}\left(h, x, t, E_{x}\right)=\sum_{\nu=0}^{\nu_{0}} \sum_{\mu} L_{\nu, \mu, 0}(h, x, t) E_{0}^{\nu} E_{-}^{\mu}
$$

and

$$
S^{(0)}\left(h, x_{-}, t, E_{x}\right)=\sum_{\nu=0}^{\nu_{0}-1} \sum_{\mu} S_{\nu, \mu, 0}(h, x, t) E_{0}^{\nu} E_{-}^{\mu}
$$

and the related difference problem
(A) $L^{(0)}\left(h, x, t, E_{x}\right) u(x)=F(x), \quad x \in R_{h}^{+} \times R_{h}^{n-1}$,
(B) $\quad S^{(0)}\left(h, x_{-}, t, E_{x}\right) u\left(0, x_{-}\right)=g\left(x_{-}\right), \quad x_{-} \in R_{h}^{n-1}$. 
Assumption 1.1. The problem in (1.15) can be solved boundedly for $u$, i.e. for any $F \in l_{2}(x), g \in l_{2}\left(x_{-}\right)$there exists a unique solution $u \in l_{2}(x)$ satisfying the estimate

$$
\|u(x)\|_{x}^{2} \leqslant K\left(\|F(x)\|_{x}^{2}+h\left\|g\left(x_{-}\right)\right\|_{x_{-}}^{2}\right)
$$

with the constant $K$ independent of $F, g, t$ and $h \leqslant h_{0}$.

In Section 6 we prove the following.

THEOREM 1.1. Assumption 1.1 is equivalent to the next three conditions.

(a) The operator $L^{(0)}\left(0, x, t, E_{x}\right)$ is elliptic of order zero, i.e. for any $(x, t) \in R^{+}$ $\times R^{n-1} \times R^{+} \cup \infty$ and $\xi=\left(\xi_{0}, \xi_{1}, \ldots, \xi_{n-1}\right)=\left(\xi_{0}, \xi_{-}\right) \in R^{n}$

$$
\operatorname{det} L^{(0)}\left(0, x, t, e^{i \xi}\right) \neq 0 \quad\left(\text { where } e^{i \xi}=e^{i \xi_{0}}, \ldots, e^{i \xi_{n-1}}\right) .
$$

(b) The row dimension of the matrices $S_{\nu, \mu, \sigma}$ is equal to the number of the roots $\kappa$ with $|\kappa|<1$ of the characteristic equation

$$
\operatorname{det} L^{(0)}\left(0, x, t, \kappa, e^{i \xi_{-}}\right)=0
$$

Due to condition (a) this number is independent of $x, t$ and $\xi_{-}$and will be denoted by $l_{0}$.

(c) For any $(x, t)$ with $x_{0}=0$ and any $\xi_{-} \in R^{n-1}$ the frozen coefficients problem

$$
\begin{array}{ll}
\text { (A) } L^{(0)}\left(0, x, t, E_{0}, e^{i \xi_{-}}\right) u\left(x_{0}\right)=0, & x_{0} \in R_{h}^{+}, \\
\text {(B) } & S^{(0)}\left(0, x_{-}, t, E_{0}, e^{i \xi_{-}}\right) u(0)=0,
\end{array}
$$

has only the trivial solution in $l_{2}\left(x_{0}\right)$.

The difference operator $L$ in (1.5) has to be consistent with the differential one in (1.1). More precisely, we have the following

Assumption 1.2. The Fourier-Laplace symbol $L\left(0, x, t, e^{i \xi}, e^{s}\right)$ of the difference operator $L\left(0, x, t, E_{x}, E_{t}\right)$ satisfies

$$
L\left(0, x, t, e^{i \xi}, e^{s}\right)=s I+i \sum_{j=0}^{n-1} A_{j}(x, t) \xi_{j}+o(|\xi|+|s|) .
$$

Assumption 1.3. The Cauchy problem for the operator $L\left(0, x, t, E_{x}, E_{t}\right)$ is well posed (provided the coefficients $L_{\nu, \mu, \sigma}(x, t)$ are defined for $x_{0}<0$ ), i.e. the solution of the initial value problem (1.8)(A), (C) with $F=0$ and $f_{\sigma} \neq 0$ satisfies the estimate

$$
\|u(x, t)\|_{x}^{2} \leqslant K e^{\eta_{0} t} \sum_{\sigma=0}^{\sigma_{0}-1}\left\|f_{\sigma}(x)\right\|_{x}^{2}
$$

where the constants $K>0, \eta_{0} \geqslant 0$ are independent of $t$ and $f_{\sigma}$.

In Section 6 we also prove the following

THEOREM 1.2. Suppose that the difference operator $L$ in (1.5) approximates the strictly hyperbolic operator in (1.1). If the symbol $L\left(0, x, t, e^{i \xi}, e^{s}\right)$ is weakly dissipative, i.e.

for any $(x, t)$ the equation $\operatorname{det} L\left(0, x, t, e^{i \xi}, e^{s}\right)=0$

has in the domain $\xi \in R^{n}, \operatorname{Re} s \geqslant 0$ the only solution

$\xi$, is $=0(\bmod 2 \pi)$,

and $L^{(0)}$ is elliptic in the sense of (1.17), then the Cauchy problem for the operator $L\left(h, x, t, E_{x}, E_{t}\right)$ is well posed. 
The well-known result of Kreiss in [9] refers, indeed, to general hyperbolic Hermitian systems but requires the difference approximation to be two-step explicit, with Hermitian coefficient matrices, dissipative of order $2 r$ and accurate of order $2 r-1$ for some positive integer $r$. Parlett in [15] has relaxed the last condition in the case of strictly hyperbolic systems by assuming the order of accuracy to be $2 r-2$.

Consider the symbol $L\left(0, x, t, e^{i \xi}, e^{s}\right)$ for small $|\xi|+|s|$. We shall introduce formal conical coordinates

$$
\xi=\xi^{\prime} \cdot r, \quad \xi^{\prime}=\left(\xi_{0}^{\prime}, \xi_{1}^{\prime}, \ldots, \xi_{n}^{\prime}\right)=\left(\xi_{0}^{\prime}, \xi_{-}^{\prime}\right), \quad s=s^{\prime} \cdot r,
$$

where $\xi^{\prime}, s^{\prime}$ and $r$ are free variables, and define a matrix function

$$
L^{\prime}\left(x, t, \xi^{\prime}, s^{\prime}, r\right)=L\left(0, x, t, e^{i \xi^{\prime} r}, e^{i s^{\prime} r}\right) / r .
$$

Because of (1.20), $L^{\prime}$ depends analytically on $\xi^{\prime}, s^{\prime}$ and $r$, and

$$
L^{\prime}\left(x, t, \xi^{\prime}, s^{\prime}, 0\right)=s^{\prime} I+i \sum A_{j}(x, t) \xi_{j}^{\prime} .
$$

We restrict $\xi^{\prime}, s^{\prime}$ and $r$ to the domain $\mathscr{D}: \delta<\left|\xi^{\prime}\right|<K_{1},\left|s^{\prime}\right|<K_{2}, 0 \leqslant r<\varepsilon$ with complex $s^{\prime}$ and real $\xi^{\prime}$ and $r$. Because of the strict hyperbolicity of the system in (1.1), for small $\varepsilon$ the eigenvalues $\lambda_{j}=f_{j}\left(x, t, \xi^{\prime}, s^{\prime}, r\right)$ of $L^{\prime}$ are distinct and depend analytically on $\left(\xi^{\prime}, s^{\prime}, r\right) \in \mathscr{D}$. The equations $f_{j}\left(x, t, \xi^{\prime}, s^{\prime}, r\right)=0$ could be solved in $s^{\prime}$ giving

$$
s^{\prime}=g_{j}\left(x, t, \xi^{\prime}, r\right), \quad j=1,2, \ldots, k,
$$

where $g_{j}\left(x, t, \xi^{\prime}, r\right)$ is an analytic function of $\xi^{\prime}, r$ for small $|r|$ and $\xi^{\prime}$ as in $\mathscr{D}$. Obviously $g_{j}\left(x, t, \xi^{\prime}, 0\right), j=1,2, \ldots, k$, are the eigenvalues of the matrix $i \Sigma A_{j}(x, t) \xi_{j}^{\prime}$, and therefore

$$
\operatorname{Re} g_{j}\left(x, t, \xi^{\prime}, 0\right)=0
$$

and for small $|r|$ the values $g_{j}\left(x, t, \xi^{\prime}, r\right), j=1,2, \ldots, k$, are distinct.

Assumption 1.4. For $(x, t)$ near the boundary $x_{0}=0$ the symbol $L\left(0, x, t, e^{i \xi}, e^{s}\right)$ satisfies

(a) the condition in (1.22)

(b) for any $\left(\xi^{\prime}\right)_{0} \in R^{n}$ with $\left(\xi_{-}^{\prime}\right)_{0} \neq 0$ there exist a neighborhood $\Omega \subset R^{n} \times R^{+}$ of the point $\left(\xi^{\prime}\right)_{0}, r=0$, and positive constants $\delta_{1}$ and $\delta_{2}$ such that for any $\left(\xi^{\prime}, r\right) \in \Omega$ we have

$$
-\delta_{1} \cdot r^{2 m_{j}-1} \leqslant \operatorname{Re} g_{j}\left(x, t, \xi^{\prime}, r\right) \leqslant-\delta_{2} r^{2 m_{j}-1}, \quad j=1,2, \ldots, k,
$$

where $m_{j}$ is a positive integer independent of $\left(\xi^{\prime}\right)_{0}, x, t$ and the neighborhood $\Omega$.

The last assumption states that near the boundary the difference operator $L$ is dissipative in the tangential direction. The order of dissipativity $2 m_{j}$ may depend on the sequential number $j$ of the eigenvalue $g_{j}$.

In order to state our main result concerning the stability of the problem in (1.8) we have to formulate the uniform Kreiss condition (UKC) for the difference problem. To do that we freeze the coefficients of $L$ and $S$ in (1.8) at a point $(x, t)=\left(0, x_{-}, t\right), h=0$, and apply to (1.8) a Fourier transform in $x_{-}$and Laplace-Fourier transform in $t$ with dual variables $\xi_{\text {- }}$ and $s$, respectively. We arrive 
at the auxiliary homogeneous problem

$$
\begin{array}{ll}
\text { (A) } L\left(0, x, t, E_{0}, e^{i \xi_{-}}, e^{s}\right) u\left(x_{0}\right)=0, \quad x_{0} \in R_{h}^{+}, \\
\text {(B) } S\left(0, x_{-}, t, E_{0}, e^{i \xi_{-}}, e^{s}\right) u(0)=0,
\end{array}
$$

with the parameters $\xi_{-}=\left(\xi_{1}, \xi_{2}, \ldots, \xi_{n-1}\right)$ and $s=i \xi_{n}+\eta$ in the domain

$$
-\pi \leqslant \xi_{j} \leqslant \pi, \quad j=1,2, \ldots, n, \eta \geqslant 0
$$

$$
\text { excluding the point } \xi_{-}=0, s=0 \text {. }
$$

Consider the characteristic equation

$$
\operatorname{det} L\left(0, x, t, \kappa, e^{i \xi_{-}}, e^{s}\right)=0 .
$$

Part (a) of Assumption 1.4 implies that for $\xi_{-}, s$ as in (1.30) the number of the roots $\kappa$ with $|\kappa|<1$ does not depend on $s$ and, because of Assumption 1.1 (see condition (b) in Theorem 1.1), this number is $l_{0}$. Thus Eq. (1.29)(A) has exactly $l_{0}$ independent solutions in $l_{2}\left(x_{0}\right)$

$$
\varphi_{j}\left(x_{0}, \xi_{-}, s\right), \quad j=1,2, \ldots, l_{0} .
$$

We may assume that these solutions are orthonormalized at the boundary points $x_{0}=\nu h, \nu=0,1, \ldots, \nu_{0}-1$. Substituting these solutions in the boundary condition (1.29)(B) we arrive at the matrix

$$
\begin{aligned}
& N\left(x_{-}, t, \xi_{-}, s\right) \\
& \quad=S\left(0, x_{-}, t, E_{0}, e^{i \xi_{-}}, e^{s}\right)\left[\varphi_{1}\left(0, \xi_{-}, s\right), \ldots, \varphi_{l_{0}}\left(0, \xi_{-}, s\right)\right] .
\end{aligned}
$$

Note that, according to condition (b) in Theorem 1.1, the matrix $N$ is square of order $l_{0}$. Then the uniform Kreiss condition states:

There exists a positive constant $\delta$ such that for any

$$
\begin{aligned}
& \left(x_{-}, t\right) \in R^{n-1} \times R^{+} \text {and any } \xi_{-}, s \text { in the domain (1.30) we } \\
& \text { have }\left|\operatorname{det} N\left(x_{-}, t, \xi_{-}, s\right)\right| \geqslant \delta .
\end{aligned}
$$

The matrix $N\left(x_{-}, t, \xi_{-}, s\right)$ is continuous at any $\left(x_{-}, t\right) \in R^{n-1} \times R^{+} \cup \infty$ and $\left(\xi_{-}, s\right)$ as in (1.30) including the points with $\eta=+\infty$. The solvability assumption 1.1 implies that the matrix $N$ is nonsingular at $\eta=+\infty$. Therefore apart from a neighborhood of the point $\xi_{-}=0, s=0(\mathrm{UKC})$ is equivalent to the inequality

$$
\begin{aligned}
& \text { (1.35) det } N\left(x_{-}, t, \xi_{-}, s\right) \neq 0, \\
& \text { where }\left(x_{-}, t\right) \in R^{n-1} \times R^{+} \cup \infty \text { and }\left(\xi_{-}, s\right) \text { are as in (1.30). } \\
& \text { In the neighborhood of }\left(\xi_{-}, s\right)=0 \text { we introduce conical coordinates } \\
& \text { (1.36) } \quad \xi_{-}=\xi_{-}^{\prime} \cdot r, \quad s=s^{\prime} \cdot r, \quad \text { where } r=\sqrt{\left|\xi_{-}\right|^{2}+|s|^{2}} .
\end{aligned}
$$

We shall show in Lemma 3.3 that the first $l$ homogeneous solutions in (1.32) (recall that $l$ is the number of positive eigenvalues of the matrix $A_{0}(x, t)$ in $(1.1)$ ), which are associated with the eigenvalue $\kappa=1$, depend continuously on $\xi_{-}^{\prime}, s^{\prime}, r$ at any point with $r=0, \xi_{-}^{\prime} \in R^{n-1}, \operatorname{Re} s^{\prime} \geqslant 0$ and $\left|\xi^{\prime}\right|^{2}+\left|s^{\prime}\right|^{2}=1$, and for $r=0$ these solutions are independent of $x_{0}$. Substituting these solutions in the boundary condition (1.29)(B), we get the $l_{0} \times l$ matrix

$$
\begin{aligned}
& N_{1}\left(x_{-}, t, \xi_{-}^{\prime}, s^{\prime}, r\right) \\
& \quad=S\left(0, x_{-}, t, E_{0}, e^{i \xi_{-}}, e^{s}\right)\left[\varphi_{1}\left(0, \xi_{-}^{\prime}, s^{\prime}, r\right), \ldots, \varphi_{l}\left(0, \xi_{-}^{\prime}, s^{\prime}, r\right)\right]
\end{aligned}
$$


such that at $r=0$ it becomes

$$
\begin{aligned}
& N_{1}\left(x_{-}, t, \xi_{-}^{\prime}, s^{\prime}, 0\right) \\
& \quad=S\left(0, x_{-}, t, 1,1,1\right)\left[\varphi_{1}\left(0, \xi_{-}^{\prime}, s^{\prime}, 0\right), \ldots, \varphi_{l}\left(0, \xi_{-}^{\prime}, s^{\prime}, 0\right)\right] .
\end{aligned}
$$

The remaining $l_{0}-l$ solutions in (1.32) depend analytically on $\left(\xi_{-}, s\right)$ and define at $\xi_{-}=0, s=0$ the matrix

$$
N_{2}\left(x_{-}, t, 0,0\right)=S\left(0, x_{-}, t, E_{0}, 1,1\right)\left[\varphi_{l+1}(0,0,0), \ldots, \varphi_{l_{0}}(0,0,0)\right]
$$

Thus, in a small neighborhood of the point $\xi_{-}=0, s=0$ (UKC) is equivalent to the condition

$$
\begin{array}{r}
\operatorname{det}\left[N_{1}\left(x_{-}, t, \xi_{-}^{\prime}, s^{\prime}, 0\right), \quad N_{2}\left(x_{-}, t, 0,0\right)\right] \neq 0, \\
\text { where }\left(x_{-}, t\right) \in R^{n-1} \times R^{+} \cup \infty, \xi_{-}^{\prime} \in R^{n-1}, \\
\qquad \operatorname{Re} s^{\prime} \geqslant 0 \text { and }\left|\xi_{-}^{\prime}\right|^{2}+\left|s^{\prime}\right|^{2}=1 .
\end{array}
$$

So, we have proved

LEMMA 1.1. (UKC) is satisfied if and only if both the determinant conditions (1.35) and (1.40) hold.

Now assume that the boundary operator in $(1.8)(B)$ is consistent with the one in (1.2). By this we mean that

$$
S\left(h, x_{-}, t, E_{x}, E_{n}\right)=\left[\begin{array}{l}
S_{1}\left(h, x_{-}, t, E_{x}, E_{n}\right) \\
S_{2}\left(h, x_{-}, t, E_{x}, E_{n}\right)
\end{array}\right],
$$

where $S_{1}$ has the row dimension $l$, and, when $E_{x}, E_{t}$ are replaced by 1 , we have

(1.42) $S_{1}\left(0, x_{-}, t, 1,1\right)$ coincides with the matrix $S\left(x_{-}, t\right)$ in (1.2)

$$
\text { and } S_{2}\left(0, x_{-}, t, 1,1\right)=0 \text {. }
$$

So $S_{1}$ approximates the original boundary condition (1.2) and $S_{2}$ represents the artificial boundary condition. Due to the partition in (1.41) we have

$$
N_{1}\left(x_{-}, t, \xi_{-}^{\prime}, s^{\prime}, 0\right)=\left[\begin{array}{c}
N_{11}\left(x_{-}, t, \xi_{-}^{\prime}, s^{\prime}, 0\right) \\
0
\end{array}\right]
$$

and

$$
N_{2}\left(x_{-}, t, 0,0\right)=\left[\begin{array}{l}
N_{21}\left(x_{-}, t, 0,0\right) \\
N_{22}\left(x_{-}, t, 0,0\right)
\end{array}\right] .
$$

We shall see again in Lemma 3.3 that the vectors $\varphi_{j}\left(0, \xi_{-}^{\prime}, s^{\prime}, 0\right), j=1, \ldots, l$, coincide with those arising in the differential problem (1.1)-(1.3) so that the matrix $N_{11}\left(x_{-}, t, \xi_{-}^{\prime}, s^{\prime}, 0\right)$ appears in the (UKC) for the problem in (1.1)-(1.3). Thus we got the following

LEMMA 1.2. If the boundary condition in (1.8)(B) is consistent with the one in (1.2), then (UKC) for the difference problem is satisfied if and only if
(a) the differential problem in (1.1)- (1.3) is well posed,
(b) det $N_{22}\left(x_{-}, t, 0,0\right) \neq 0$,
(c) condition (1.35) is satisfied. 
Actually, condition (b) in the above lemma claims that for $\left(\xi_{-}, s\right)=0$ Eq. (1.29)(A) has no nontrivial solutions in $l_{2}\left(x_{0}\right)$ satisfying the artificial boundary conditions in (1.29)(B), whereas condition (c) means that for $\left(\xi_{-}, i s\right) \neq 0 \bmod 2 \pi$ and $\operatorname{Re} s \geqslant 0$ Eq. (1.28)(A) has no such solutions satisfying all the boundary conditions in (1.28)(B).

The main result of this work is

THEOREM 1.3. (UKC) is sufficient and, in the case of constant coefficients, necessary for the stability of the problem in (1.8).

Our definition of stability allows for bounded exponential growths of the solution. However, if the operators $L$ and $S$ in (1.8) do not depend on $x, t$ and $h$, the constant $\eta_{0}$ in Definition 1.1 becomes zero so that there are no exponentially growing solutions for $F \in l_{2}(x, t)$ and $g \in l_{2}\left(x_{-}, t\right)$.

As in [5] (see Theorem 5.4), Theorem 1.3 may be easily generalized to the case with two boundaries.

THEOREM 1.4. Consider the difference equation (1.8)(A) in the strip $0 \leqslant x_{0} \leqslant 1$, $x_{-} \in R^{n-1}, t \geqslant 0$ with zero initial conditions and with boundary conditions of the type (1.8)(B) at $x_{0}=0$ and $x_{0}=1$. The above difference problem is stable provided the corresponding left and right quarter-space problems (which we get by removing one boundary to infinity) are stable.

Note that a solution of a stable problem in the strip may grow exponentially even if the coefficients of $L$ and $S$ are constant.

Unfortunately, it is very hard to check whether (UKC), and, especially, condition (1.35) for $\xi_{-} \neq 0$, is satisfied. Kreiss in [10] has suggested to add sufficient amount of dissipation in the tangential direction to make the multidimensional problem stable. Indeed, replace the problem in (1.8) by

$$
\begin{aligned}
& \text { (A) } L\left(h, x, t, E_{x}, E_{n}\left(I-K \varphi\left(x_{0}\right) \Delta^{k}\right)\right) u(x, t)=h \cdot F(x, t), \\
& \text { (B) } S\left(h, x, t, E_{x}, E_{n}\left(I-K \Delta^{k}\right)\right) u\left(0, x_{-}, t\right)=g\left(x_{-}, t\right),
\end{aligned}
$$

with zero initial condition. Here $\Delta=\sum_{j=1}^{n-1}\left(E_{j}+E_{j}^{-1}-2\right), \varphi\left(x_{0}\right)$ is a cut-off function which is one near the boundary $x_{0}=0$ and decreases to zero apart of it, $k$ is a positive integer and $K$ is a positive constant.

THEOREM 1.5. Let the problem in (1.8) satisfy the conditions:

(a) Assumptions 1.1-1.3 are valid;

(b) problem (1.8) is consistent with a well-posed problem (1.1)-(1.3);

(c) for $\xi_{-}=0$ and $(x, t)$ at the boundary, including $(x, t)=\infty$, condition (1.22) is satisfied with regard to $\xi_{0}$ and $s$;

(d) $(U K C)$ is satisfied for $\xi_{-}=0$ and $\eta \geqslant 0$.

Then for any $k$ there exists a constant $K$ such that the problem in (1.45) is stable.

The proof of the theorem is trivial. First, note that problem (1.45) satisfies the conditions of Theorem 1.3. Thus we have to check only (UKC) for problem (1.45). Let $N(\xi, s)$ be the matrix defined in (1.33) for problem $(1.8)$ and $\tilde{N}(\xi, s)$ the 
corresponding matrix for problem (1.45). Then

$$
\tilde{N}\left(\xi_{-}, s\right)=N\left(\xi_{-}, \tilde{s}\right), \quad \text { where } \tilde{s}=s+\ln \left[1+K\left(\sum_{j=1}^{n-1} 4 \sin ^{2}\left(\xi_{j} / 2\right)\right)^{k}\right] \text {. }
$$

Condition (d) means that det $N(0, s) \neq 0$ for any $\eta \geqslant 0$. Also, because of Assumption 1.1 , det $N\left(\xi_{-}, s\right) \neq 0$ for $\eta$ greater than some constant $K_{0}$ and any $\xi_{-}$. In view of Lemma 1.2, condition (b) and the fact that $\operatorname{det} N(0, s) \neq 0$ imply that $\operatorname{det} N\left(\xi_{-}, s\right) \neq 0$ also for $\left|\xi_{-}\right|<\varepsilon$ and any $\eta \geqslant 0$. Now we choose $K$ big enough such that for $\left|\xi_{-}\right| \geqslant \varepsilon$,

$$
\ln \left[1+K\left(\sum_{j=1}^{n-1} 4 \sin ^{2}\left(\xi_{j} / 2\right)\right)^{k}\right] \geqslant K_{0} \text {. Q.E.D. }
$$

The scheme in (1.45) includes negative powers of the operator $I-K \Delta^{k}$ if the forms (1.5) and (1.6) of the operators $L$ and $S$ are used. One could, of course, multiply $L$ and $S$ by $E_{n}^{\sigma_{0}}$ to cancel the negative powers of $E_{n}$. In that case the scheme in (1.45) will become implicit. There is, however, no way of adding sufficient amount of dissipation in an explicit form without violating the stability of the scheme.

2. Preliminaries. First, let us point out that the stability of the problem in (1.8) does not depend on the terms of order $h$ in the operators $L$ and $S$. To prove that one can use the same type of arguments as in [5, Theorem 4.3]. The same is true for the results in Theorems 1.1 and 1.2. Therefore we shall assume that $L$ and $S$ do not depend explicitly on the increment $h$ and omit $h$ from the list of variables in $L$ and $S$.

Next, we shall show that if Assumption 1.1 is satisfied, all we have to prove in the sufficiency part of Theorem 1.3 is the a priori estimate (1.12) for bounded values of $\eta h$. Indeed, substitute in (1.8)

$$
u(x, t)=e^{\eta t} u_{\eta}(x, t), \quad h F(x, t)=e^{\eta t} F_{\eta}(x, t), \quad g\left(x_{-}, t\right)=e^{\eta t} g_{\eta}\left(x_{-}, t\right) .
$$

Then we get

$$
\begin{aligned}
& \text { (A) } L_{\eta} u_{\eta}=F_{\eta}, \\
& \text { (B) } S_{\eta} u_{\eta}=g_{\eta},
\end{aligned}
$$

where

$$
\begin{aligned}
L_{\eta} & =\sum_{\sigma=0}^{-\sigma_{0}} e^{\sigma h \eta} L^{(\sigma)} E_{n}^{\sigma}, & S_{\eta} & =\sum_{\sigma=0}^{-\sigma_{0}} e^{\sigma h \eta} S^{(\sigma)} E_{n}^{\sigma}, \\
L^{(\sigma)} & =\sum_{\nu} \sum_{\mu} L_{\nu, \mu, \sigma} E_{0}^{\nu} E_{-}^{\mu}, & S^{(\sigma)} & =\sum_{\nu} \sum_{\mu} S_{\nu, \mu, \sigma} E_{0}^{\nu} E_{-}^{\mu}
\end{aligned}
$$

Estimate (1.12) in new variables takes the form

$$
\begin{aligned}
& \frac{\eta h}{1+\eta h}\left\|u_{\eta}\right\|_{x, t}^{2}+h \sum_{\nu=0}^{\nu_{0}-1}\left\|u_{\eta}\left(x_{0}=\nu h\right)\right\|_{x_{-}, t}^{2} \\
& \quad \leqslant K\left(\frac{1+\eta h}{\eta h}\left\|F_{\eta}\right\|_{x, t}^{2}+h\left\|g_{\eta}\right\|_{x_{-}, t}^{2}\right)
\end{aligned}
$$


Denote the pair of operators $\left(L_{\eta}, S_{\eta}\right)$ by $\mathcal{L}_{\eta}$. We consider $\mathcal{L}_{\eta}$ as an operator which maps grid functions $u_{\eta} \in l_{2}(x, t)$ into pairs $\left(F_{\eta}, g_{\eta}\right), F_{\eta} \in l_{2}(x, t), g_{\eta} \in l_{2}\left(x_{-}, t\right)$ with the norm

$$
\left\|\varrho_{\eta} u_{\eta}\right\|^{2}=\left\|F_{\eta}\right\|^{2}+h\left\|g_{\eta}\right\|^{2} .
$$

For large $h \eta$ the operator $\mathcal{L}_{\eta}$ approximates the operator

$$
\varrho^{(0)}=\left(L^{(0)}, S^{(0)}\right): l_{2}(x, t) \rightarrow\left(l_{2}(x, t), l_{2}\left(x_{-}, t\right)\right)
$$

which, according to Assumption 1.1, is an isomorphism with a bounded inverse $\left\|\left(\mathcal{L}^{(0)}\right)^{-1}\right\| \leqslant K$. Therefore, for large $h \eta$ (say $\left.h \eta>K_{0}\right) \mathcal{L}_{\eta}$ is an isomorphism too and $\left\|\mathcal{L}_{\eta}^{-1}\right\| \leqslant K$. The last estimate is equivalent to (2.2) for large $\eta h$. Now suppose that the a priori estimate (2.2) holds for any $\eta>\eta_{0} \geqslant 0$ and $h \leqslant h_{0}$. Fixing $h$ and considering $\eta$ in the domain $\eta>\eta_{1}>\eta_{0}$ we get

$$
\left\|u_{\eta}\right\|_{x, t}^{2} \leqslant K_{1}\left\|\mathcal{L}_{\eta} u_{\eta}\right\|_{x, t}^{2}
$$

where $K_{1}$ depends on $h$ and $\eta_{1}$ but not on $u_{\eta} \in l_{2}(x, t)$. Since $\mathcal{L}_{\eta}$ is an isomorphism for large $\eta$, by continuity arguments we derive from (2.3) that $\mathcal{L}_{\eta}$ is an isomorphism for any $\eta \geqslant \eta_{1}$ and therefore for any $\eta>\eta_{0}$. Finally, let $F \in e^{\eta t} l_{2}(x, t), g \in$ $e^{\eta t} l_{2}\left(x_{-}, t\right)$, and define $u \in e^{\eta t} l_{2}(x, t)$ by

$$
u=e^{\eta t} \mathcal{L}_{\eta}^{-1}\left(e^{-\eta t} h^{-1} F, e^{-\eta t} g\right) .
$$

If $F$ and $g$ vanish for $t<\sigma_{0} h$, then $u$ vanishes too, thus providing a solution for (1.8) with zero initial data. Indeed, we could replace the weight $e^{-\eta t}$ in Definition 1.1 by $e^{-\eta\left(t-\sigma_{0} h\right)}$ without affecting estimate (1.12). Then, where $u \neq 0$ for some $t<\sigma_{0} h$, estimate (1.12) would be violated as $\eta \rightarrow \infty$.

Finally, we shall linearize problem (1.8) in the direction $x_{0}$. Introduce grid vector-functions

$$
\tilde{u}(x, t)=\left(u(x, t), E_{0} u(x, t), \ldots, E_{0}^{\nu_{0}-1} u(x, t)\right)^{\prime}
$$

and

$$
\tilde{F}(x, t)=(0,0, \ldots, F(x, t))^{\prime},
$$

and difference operators

$$
\begin{gathered}
\tilde{S}\left(x_{-}, t, E_{-}, E_{n}\right)=\left[\tilde{S}_{0}, \tilde{S}_{1}, \ldots, \tilde{S}_{\nu_{0}-1}\right] \\
\tilde{L}\left(x, t, E_{x}, E_{n}\right)=A\left(x, t, E_{-}, E_{n}\right) E_{0}+B\left(x, t, E_{-}, E_{n}\right),
\end{gathered}
$$

where

$$
\begin{gathered}
B=\left[\begin{array}{ccccc}
0 & -I & 0 & \cdots & 0 \\
0 & 0 & -I & & \vdots \\
\vdots & & & \ddots & -I \\
\tilde{L}_{0} & \tilde{L}_{1} & \cdots & & \tilde{L}_{\nu_{0}-1}
\end{array}\right], \quad A=\operatorname{diag}\left(I, I, \ldots, I, \tilde{L}_{\nu_{0}}\right), \\
\tilde{L}_{\nu}=\sum_{\sigma=0}^{-\sigma_{0}} \sum_{\mu} L_{\nu, \mu, \sigma}(x, t) E_{-}^{\mu} E_{n}^{\sigma}
\end{gathered}
$$


and

$$
\tilde{S}_{\nu}=\sum_{\sigma=0}^{-\sigma_{0}} \sum_{\mu} S_{\nu, \mu, \sigma}\left(x_{-}, t\right) E_{-}^{\mu} E_{n}^{\sigma} .
$$

Then Eqs. (1.8)(A) and (1.8)(B) extended to $t<\sigma_{0} h$ could be rewritten as
(A) $\quad \tilde{L}\left(x, t, E_{x}, E_{n}\right) \tilde{u}(x, t)=h \tilde{F}(x, t), \quad(x, t) \in R_{h}^{+} \times R_{h}^{n}$,
(B) $\tilde{S}\left(x_{-}, t, E_{-}, E_{n}\right) \tilde{u}\left(0, x_{-}, t\right)=g\left(x_{-}, t\right), \quad\left(x_{-}, t\right) \in R_{h}^{n}$.

The operator $\tilde{L}$ is connected with $L$ by the equivalence

$$
D_{2}\left(x, t, E_{x}, E_{n}\right) \tilde{L}\left(x, t, E_{x}, E_{n}\right) D_{1}\left(E_{0}\right)=\left[\begin{array}{cc}
L\left(x, t, E_{x}, E_{n}\right) & 0 \\
0 & I_{k\left(\nu_{0}-1\right)}
\end{array}\right],
$$

where

$$
D_{1}=\left[\begin{array}{ccccc}
I & 0 & 0 & \cdots & 0 \\
E_{0} & I & 0 & & 0 \\
E_{0}^{2} & E_{0} & I & & \vdots \\
\vdots & & & \ddots & 0 \\
E_{0}^{\nu_{0}-1} & \cdots & E_{0}^{2} & E_{0} & I
\end{array}\right], \quad D_{2}=\left[\begin{array}{ccccc}
C_{\nu_{0}-1} & C_{\nu_{0}-2} & \cdots & C_{1} & I \\
-I & 0 & & 0 & 0 \\
0 & -I & & & \\
\vdots & & \ddots & & \vdots \\
0 & \cdots & & -I & 0
\end{array}\right] \text {, }
$$

and

(2.14) $C_{1}=\tilde{L}_{\nu_{0}} E_{0}+\tilde{L}_{\nu_{0}-1}, \quad C_{\nu+1}=C_{\nu} E_{0}+\tilde{L}_{\nu_{0}-1-\nu} \quad$ for $\nu=1,2, \ldots, \nu_{0}-2$

Note that $D_{1}$ and $D_{2}$ are invertible operators with the inverses

$$
D_{1}^{-1}=\left[\begin{array}{cccc}
I & 0 & \cdots & 0 \\
-E_{0} & I & & \\
& & \ddots & \vdots \\
0 & & I & 0 \\
\vdots & & & \\
0 & \cdots & -E_{0} & I
\end{array}\right], \quad D_{2}^{-1}=\left[\begin{array}{ccccc}
0 & -I & 0 & \cdots & 0 \\
& 0 & -I & & \\
\vdots & & \ddots & & \vdots \\
I & C_{\nu_{0}-1} & \cdots & & C_{1}
\end{array}\right] \text {, }
$$

and the norms of the operators $D_{1}, D_{2}, D_{1}^{-1}$, and $D_{2}^{-1}$ in the spaces $e^{\eta t} l_{2}(x, t)$ are bounded uniformly in $\eta \geqslant 0$. If $\tilde{u}$ and $\tilde{F}$ are defined by (2.4) and (2.5) then estimate (1.12) is equivalent to the estimate

$$
\begin{aligned}
\frac{\eta}{1+\eta h}\|\tilde{u}(x, t)\|_{x, t, \eta}^{2} & +\left\|\tilde{u}\left(0, x_{-}, t\right)\right\|_{x_{-}, t, \eta}^{2} \\
& \leqslant K\left(\frac{1+\eta h}{\eta}\|\tilde{F}(x, t)\|_{x, t, \eta}^{2}+\left\|g\left(x_{-}, t\right)\right\|_{x_{-}, t, \eta}^{2}\right) .
\end{aligned}
$$

Now suppose that $\tilde{u}$ in (2.11) is an arbitrary grid function belonging to $e^{\eta t} l_{2}(x, t)$, and $\tilde{F}$ and $g$ are given by Eqs. (2.11)(A) and (B). Define the grid vector-functions

$$
\begin{array}{ll}
u=(I, 0,0, \ldots, 0) D_{1}^{-1} \tilde{u}, & F=(I, 0, \ldots, 0) D_{2} \tilde{F}, \\
v=D_{1}^{-1} \tilde{u}-(u, 0, \ldots, 0)^{\prime}, & G=D_{2} \tilde{F}-(F, 0, \ldots, 0)^{\prime},
\end{array}
$$


where the unit and zero matrices are of order $k$. Then equivalence (2.12) implies

$$
v=h G
$$

and

$$
\left\{\begin{array}{l}
L u(x, t)=h F(x, t) \\
S u\left(0, x_{-}, t\right)=g-\tilde{S} D_{1} v\left(0, x_{-}, t\right)=g-h \tilde{S} D_{1} G\left(0, x_{-}, t\right) .
\end{array}\right.
$$

Using (2.18), (2.19) and the boundedness of $D_{1}$ and $D_{2}$ one can easily show that the a priori estimate (1.12) implies estimate (2.16). Thus we have proved

LEMMA 2.1. Estimate (1.12) for problem (1.8) is equivalent to estimate (2.16) for problem (2.11) with the same constants $\eta_{0}$ and $h_{0}$.

An analogous result related to the solvability assumption 1.1 holds for problem (1.15) and its linearization. Namely, let

$$
\begin{aligned}
& \text { (A) } \tilde{L}^{(0)}\left(x, t, E_{x}\right) \tilde{u}(x)=\tilde{F}(x), \\
& \text { (B) } \tilde{S}^{(0)}\left(x, t, E_{-}\right) \tilde{u}\left(0, x_{-}\right)=g\left(x_{-}\right),
\end{aligned}
$$

be the linearization of problem (1.15) in the direction $x_{0}$.

Lemma 2.2. Assumption 1.1 and conditions of Theorem 1.1 for problem (1.15) are equivalent to the same assumption and conditions for problem (2.20).

Therefore, with regard to Theorems 1.1 and 1.3 we restrict ourselves to the linearized problems (2.11) and (2.20). For ease of notation we also remove the symbol ${ }^{\sim}$ from $\tilde{u}$ and $\tilde{F}$ in (2.11), (2.16) and (2.20).

3. The Case of Constant Coefficients. We consider problem (2.11) with the coefficients of $\tilde{L}$ and $\tilde{S}$ frozen at some boundary point $(x, t)=\left(0, x_{-}, t\right)$. Apply to this problem the Fourier transform in $x_{-}$with the dual variable $\xi_{-}=$ $\left(\xi_{1}, \xi_{2}, \ldots, \xi_{n-1}\right)$ and Laplace-Fourier transform in $t$ with the dual variable $s=i \xi_{n}$ $+\eta$. We arrive at the difference problem in the direction $x_{0}$ depending on the parameters $\xi_{-}$and $s$

$$
\begin{aligned}
& \text { (A) } \tilde{L}\left(E_{0}, e^{i \xi_{-}}, e^{s}\right) \hat{u}\left(x_{0}, \xi_{-}, s\right)=h \hat{F}\left(x_{0}, \xi_{-}, s\right), \\
& \text { (B) } \tilde{S}\left(e^{i \xi_{-}}, e^{s}\right) \hat{u}\left(0, \xi_{-}, s\right)=\hat{g}\left(\xi_{-}, s\right),
\end{aligned}
$$

where

$$
\hat{u}\left(x_{0}, \xi_{-}, s\right)=\sum_{\left(x_{-}, t\right) \in R_{h}^{n}} u\left(x_{0}, x_{-}, t\right) e^{-\left(i x_{-} \xi_{-}+s t\right) / h}
$$

and $\hat{g}$ and $\hat{F}$ are defined in a similar way. We shall use the notations

$$
\xi=\left(\xi_{-}, \xi_{n}\right), \quad \zeta=(\xi, \eta)
$$

and often identify $\zeta$ with the pair $\left(\xi_{-}, s\right)$. We shall also write $\tilde{L}\left(E_{0}, \zeta\right), \tilde{S}(\zeta)$ and $L\left(E_{0}, \zeta\right)$ instead of the expressions in (3.1) and (1.29). Removing the sign from $\hat{u}, \hat{F}$ and $\hat{g}$, omitting the parameters $\xi_{-}, s$ in these functions and replacing $h F$ by $F$ we rewrite (3.1) as

$$
\begin{aligned}
& \text { (A) } \tilde{L}\left(E_{0}, \zeta\right) u\left(x_{0}\right)=\left(A(\zeta) E_{0}+B(\zeta)\right) u\left(x_{0}\right)=F \\
& \text { (B) } \tilde{S}(\zeta) u(0)=g
\end{aligned}
$$


Then estimate (2.16) in the domain $0<\eta h \leqslant K_{0}$ is equivalent to the estimate

$$
\eta\left\|u\left(x_{0}\right)\right\|_{x_{0}}^{2}+h|u(0)|^{2} \leqslant K\left(\eta^{-1}\left\|F\left(x_{0}\right)\right\|_{x_{0}}^{2}+h|g|^{2}\right)
$$

in the domain $0<\eta \leqslant K_{0}$ with $K$ independent on $\zeta$ and $u$.

3.1. The Normal Form of the Operator $\tilde{L}\left(E_{0}, \zeta\right)$. As we have already pointed out, the matrix $\tilde{L}_{\nu_{0}}(\zeta)$ and therefore $A(\zeta)$ may be singular. Nevertheless, there exists an appropriate normal form for the operator $\tilde{L}\left(E_{0}, \zeta\right)$. Before we study this form it is worthwhile to present some facts concerning so-called regular pencils of matrices. By the last we mean a linear matrix polynomial $C(\kappa)=A \kappa+B$ such that $A$ and $B$ are square matrices of order $n$ and the characteristic equation

$$
\operatorname{det} C(\kappa)=0
$$

does not vanish identically in $\kappa$. Let $\kappa_{0}$ be a solution of (3.6) and $\varphi\left(\kappa_{0}\right) \in \operatorname{Ker} C\left(\kappa_{0}\right)$. Then $\kappa_{0}$ is called a finite eigenvalue and $\varphi\left(\kappa_{0}\right)$ the corresponding eigenvector of the pencil $C(\kappa)$. If the matrix $A$ is singular, we call $\kappa=\infty$ the infinite eigenvalue and $\varphi(\infty) \in \operatorname{Ker} A$ the corresponding eigenvector. Let $\Gamma_{1}, \Gamma_{2}, \ldots, \Gamma_{m}$ be positive oriented disjoint Jordan contours in $\mathbf{C}$ and $\Gamma_{\infty}$ be a negative oriented one surrounding all the contours above. We assume that none of the eigenvalues of the pencil lie on the contours as well as in the exterior to $\Gamma_{j}, j=1,2, \ldots, m$, and in the interior to $\Gamma_{\infty}$. Denote by $\Gamma_{0}$ the positive oriented contour obtained from $\Gamma_{\infty}$ by mapping $\kappa \rightarrow \kappa^{-1}$. Define linear operators

$$
\begin{gathered}
P_{j}=(2 \pi i)^{-1} \oint_{\Gamma_{j}}(A \kappa+B)^{-1} A d \kappa, \quad j=1, \ldots, m, \\
P_{\infty}=(2 \pi i)^{-1} \oint_{\Gamma_{0}}(A+B \kappa)^{-1} B d \kappa .
\end{gathered}
$$

Using the resolvent equation

$$
C^{-1}(\kappa) A C^{-1}(\lambda)=(\lambda-\kappa)^{-1}\left(C^{-1}(\kappa)-C^{-1}(\lambda)\right)
$$

one can prove by standard methods that $P_{j}, j=1, \ldots, m$, are mutually orthogonal projectors. Applying the transformation $\kappa \rightarrow 1 / \kappa$ we get

$$
P_{\infty}=-(2 \pi i)^{-1} \oint_{\Gamma_{\infty}}(A \kappa+B)^{-1} B \kappa^{-1} d \kappa=I+(2 \pi i)^{-1} \oint_{\Gamma_{\infty}}(A \kappa+B)^{-1} A d \kappa .
$$

Therefore $P_{1}+P_{2}+\cdots+P_{m}+P_{\infty}=I$ and $P_{\infty}$ is also a projector orthogonal to $P_{1}, \ldots, P_{m}$. Let $\Omega_{j}$ be an open set including $\Gamma_{j}$ and its interior. Denote by $\Phi\left(\Omega_{j}\right)$ the space of $n$-dimensional complex vector-functions $\varphi(\kappa)$ analytic in $\Omega_{j}$. Define an operator $Q_{j}: \Phi\left(\Omega_{j}\right) \rightarrow \mathrm{C}^{n}$ by

$$
Q_{j}(\varphi)=(2 \pi i)^{-1} \oint_{\Gamma_{j}} C^{-1}(\kappa) \varphi(\kappa) d \kappa .
$$

Obviously $Q_{j}(A \varphi)=P_{j} \varphi$ for $\varphi(\kappa)$ constant, so that $\operatorname{Im} P_{j} \subset \operatorname{Im} Q_{j}$. On the other hand, applying the same standard method as in the proof of the equality $P_{j}^{2}=P_{j}$, one can show that $P_{j} Q_{j}(\varphi)=Q_{j}(\varphi)$. Therefore $\operatorname{Im} Q_{j} \subset \operatorname{Im} P_{j}$ and we get

$$
\operatorname{Im} Q_{j}=\operatorname{Im} P_{j}
$$


Let the dimension of $\operatorname{Im} P_{j}$ be $d_{j}$. There is some $n \times d_{j}$ matrix-function $\Psi_{j}(\kappa)$ analytic in $\Omega_{j}$ such that the columns of the matrix $X_{j}=Q_{j}\left(\Psi_{j}\right)$ form a basis in $\operatorname{Im} P_{j}$. For any $\varphi \in \Phi\left(\Omega_{j}\right)$ the following identity holds:

$$
B Q_{j}(\varphi)=(2 \pi i)^{-1} \oint_{\Gamma_{j}}(C(\kappa)-A \kappa) C^{-1}(\kappa) \varphi(\kappa) d \kappa=-A Q_{j}(\kappa \varphi) .
$$

Therefore $A Q_{j}\left(\kappa \Psi_{j}\right)+B X_{j}=0$, and representing $Q_{j}\left(\kappa \Psi_{j}\right)$ as

$$
Q_{j}\left(\kappa \Psi_{j}\right)=X_{j} M_{j},
$$

where $M_{j}$ is a square matrix of order $d_{j}$, we get

$$
A X_{j} M_{j}+B X_{j}=0, \quad j=1,2, \ldots, m .
$$

Similarly, for $j=\infty$ we get the equality

$$
A X_{\infty}+B X_{\infty} M_{\infty}=0,
$$

where the columns of $X_{\infty}$ form a basis in $\operatorname{Im} P_{\infty}$. Combining (3.11) and (3.12), we obtain as in [4] the main equivalence

$$
(A \kappa+B) X=T \cdot \operatorname{diag}\left(\kappa I-M_{1}, \ldots, \kappa I-M_{m},-\kappa M_{\infty}+I\right),
$$

where

$$
X=\left(X_{1}, \ldots, X_{m}, X_{\infty}\right) \text { and } T=\left(A X_{1}, \ldots, A X_{m}, B X_{\infty}\right) .
$$

Obviously, the matrix $X$ is invertible and, due to (3.13) and the regularity of $C(\kappa)$, the matrix $T$ is invertible too. Finally, we claim that the eigenvalues of $M_{j}$, $j=1,2, \ldots, m$, are located in the interior to $\Gamma_{j}$ and coincide there with the eigenvalues of $C(\kappa)$ (for $j=\infty$ the similar result relates to the contour $\Gamma_{0}$ and the eigenvalues of $A+B \kappa)$. Moreover, if $\kappa_{j}$ is an eigenvalue of $M_{j}$, then

$$
\operatorname{dim} \operatorname{Ker}\left(A \kappa_{j}+B\right)=\operatorname{dim} \operatorname{Ker}\left(\kappa_{j} I-M_{j}\right) .
$$

Indeed, if $\kappa_{j}$ is an eigenvalue of $M_{j}$ with the eigenvector $\psi\left(\kappa_{j}\right)$, then (3.11) implies that $\kappa_{j}$ is also an eigenvalue of $C(\kappa)$ with the eigenvector $\varphi\left(\kappa_{j}\right)=X_{j} \psi\left(\kappa_{j}\right)$. Suppose that $\kappa_{j}$ is not in the interior to $\Gamma_{j}$. Then, integrating the identity

$$
\varphi\left(\kappa_{j}\right)\left(\kappa-\kappa_{j}\right)^{-1}=C^{-1}(\kappa) A \varphi\left(\kappa_{j}\right)
$$

along the contour $\Gamma_{j}$, we would get

$$
0=P_{j}\left(X_{j} \psi\left(\kappa_{j}\right)\right)=X_{j} \psi\left(\kappa_{j}\right) .
$$

The remaining part of our claim follows immediately from equivalence (3.13).

Now let us return to the operator $\tilde{L}\left(E_{0}, \zeta\right)$ and consider the characteristic equation

$$
\operatorname{det} \tilde{L}(\kappa, \zeta)=\operatorname{det} L(\kappa, \zeta)=0,
$$

where the first equality follows from equivalence (2.12). For $\zeta$ in the domain

$$
-\pi \leqslant \xi_{j} \leqslant \pi, \quad j=1,2, \ldots, n, \quad \eta \geqslant 0,
$$

the characteristic equation, as it follows from part (a) of Assumption 1.4, is regular in $\kappa$. Moreover, for nonzero $\zeta$ in (3.17) there is no eigenvalue $\kappa$ on the unit circle $|\kappa|=1$ and for $\zeta=0$ the only such eigenvalue is $\kappa=1$. Assumption 1.2 implies

$$
L(\kappa, 0)=A_{0}(\kappa-1)+o(\kappa-1) \text {. }
$$


Therefore $\kappa=1$ is an eigenvalue of multiplicity $k$ of the pencil $\tilde{L}(\kappa, 0)$ with $k$ independent eigenvectors spanning the space

$$
\operatorname{Ker} \tilde{L}(1,0)=\left\{(u, u, \ldots, u)^{\prime} \mid u \in R^{k}\right\} .
$$

Fix a point $\zeta_{0}=\left((\xi)_{0}, \eta_{0}\right) \neq 0$ belonging to the domain in (3.17) and denote by $\Omega\left(\zeta_{0}\right)$ a small neighborhood of $\zeta_{0}$ in $R^{n+1}$. Let $\Gamma_{0}$ be a positive oriented circle $|\kappa|=1-\varepsilon$ such that the eigenvalues $\kappa$ of $\tilde{L}\left(\zeta_{0}, \kappa\right)$ are either inside $\Gamma_{0}$ or outside the circle $|\kappa|=(1-\varepsilon)^{-1}$. Then for small $\Omega\left(\zeta_{0}\right)$ the projectors

$$
\begin{aligned}
& P_{0}(\zeta)=(2 \pi i)^{-1} \oint_{\Gamma_{0}}(A(\zeta) \kappa+B(\zeta))^{-1} A(\zeta) d \kappa, \\
& P_{\infty}(\zeta)=(2 \pi i)^{-1} \oint_{\Gamma_{0}}(A(\zeta)+B(\zeta) \kappa)^{-1} B(\zeta) d \kappa,
\end{aligned}
$$

and the corresponding matrices $X_{j}(\zeta), M_{j}(\zeta), j=0, \infty$, depend analytically on $\zeta \in \Omega\left(\zeta_{0}\right)$. By choosing an appropriate $X_{j}(\zeta)$ we may even assume that

$$
M_{j}^{*}(\zeta) M_{j}(\zeta) \leqslant(1-\varepsilon) I, \quad j=0, \infty
$$

Equivalence (3.13) in our case becomes

$$
\tilde{L}(\kappa, \zeta) X(\zeta)=T(\zeta)\left(\begin{array}{cc}
\kappa I-M_{0}(\zeta) & 0 \\
0 & -\kappa M_{\infty}(\zeta)+I
\end{array}\right)
$$

where

$$
X=\left(X_{0}, X_{\infty}\right), \quad T=\left(A X_{0}, B X_{\infty}\right) .
$$

Now consider the case $\zeta_{0}=0$. In addition to the above projectors and matrices $X_{j}$, $M_{j}, j=0, \infty$ we also look at the projector

$$
P_{1}(\zeta)=(2 \pi i)^{-1} \oint_{\Gamma_{1}}(A(\zeta) \kappa+B(\zeta))^{-1} A(\zeta) d \kappa,
$$

where $\Gamma_{1}$ is a small positive oriented circle around $\kappa=1$, and at the associated matrices $X_{1}(\zeta)$ and $M_{1}(\zeta)$. Due to (3.19) and (3.15)

$$
X_{1}(0)=(I, I, \ldots, I)^{\prime} \text { and } M_{1}(0)=I .
$$

Denote

$$
\begin{aligned}
X_{f}=\left(X_{0}, X_{1}\right), \quad X & =\left(X_{f}, X_{\infty}\right), \quad T=\left(A X_{f}, B X_{\infty}\right), \\
M_{f} & =\operatorname{diag}\left(M_{0}, M_{1}\right) .
\end{aligned}
$$

Then instead of equivalence (3.22) we get

$$
\tilde{L}(\kappa, \zeta) X(\zeta)=T(\zeta) \operatorname{diag}\left(\kappa I-M_{f}(\zeta),-\kappa M_{\infty}(\zeta)+I\right) .
$$

In order to study the block form of the matrix $M_{1}(\zeta)$ in the neighborhood of the point $\zeta_{0}=0$, we introduce conical coordinates

$$
r=|\zeta|, \quad \zeta=\zeta^{\prime} \cdot r, \quad \kappa=1+\kappa^{\prime} r
$$

where $\zeta^{\prime}$ is identified with $\left(\xi^{\prime}, \eta^{\prime}\right)$ as well as with $\left(\xi_{-}^{\prime}, s^{\prime}\right)$, and define matrixfunctions

$$
L^{\prime}\left(\kappa^{\prime}, \zeta^{\prime}, r\right)=L\left(1+\kappa^{\prime} r, \zeta^{\prime} r\right) / r, \quad M^{\prime}\left(\kappa^{\prime}, \zeta^{\prime}, r\right)=\left(M_{1}\left(1+\kappa^{\prime} r, \zeta^{\prime} r\right)-I\right) / r .
$$


Note that $L^{\prime}\left(\kappa^{\prime}, \zeta^{\prime}, r\right)$ and $M^{\prime}\left(\kappa^{\prime}, \zeta^{\prime}, r\right)$ depend analytically on $\kappa^{\prime}, \zeta^{\prime}, r$ for bounded $\kappa^{\prime}$ and $\zeta^{\prime}$ and small $r$. Using the equivalence (2.12) we rewrite (3.11) for $j=1$ as

$$
\begin{aligned}
\left(\begin{array}{cc}
r L^{\prime}\left(\kappa^{\prime}, \zeta^{\prime}, r\right) & 0 \\
0 & I_{k\left(\nu_{0}-1\right)}
\end{array}\right) D_{1}^{-1}(\kappa) & X_{1}(\zeta) \\
& =r D_{2}(\kappa, \zeta) A(\zeta) X_{1}(\zeta)\left(\kappa^{\prime} I-M^{\prime}\left(\zeta^{\prime}, r\right)\right)
\end{aligned}
$$

Comparing the first $k$ rows in the above equality, and dividing them by $r$ we get at $r=0$ :

$$
L^{\prime}\left(\kappa^{\prime}, \zeta^{\prime}, 0\right)=\left[C_{\nu-1}+C_{\nu-2}+\cdots+C_{1}+\tilde{L}_{\nu_{0}}\right]\left(\kappa^{\prime} I-M^{\prime}\left(\zeta^{\prime}, 0\right)\right) .
$$

Then Assumption 1.2 implies that the matrix in the brackets is $A_{0}$ and the matrix $M^{\prime}\left(\zeta^{\prime}, 0\right)$ equals to

$$
M^{\prime}\left(\zeta^{\prime}, 0\right)=-A_{0}^{-1}\left(s^{\prime} I+i \sum_{j=1}^{n-1} \xi_{j}^{\prime} A_{j}\right) .
$$

3.2. Construction of the Symmetrizer for the Matrix $M_{1}(\zeta)$. We fix a point $\zeta_{0}^{\prime}=\left(\left(\xi^{\prime}\right)_{0}, \eta_{0}^{\prime}\right)$ with $\left|\zeta_{0}^{\prime}\right|=1$ and $\eta_{0}^{\prime} \geqslant 0$, and study the matrix $M^{\prime}\left(\zeta^{\prime}, r\right)$ in a neighborhood $\Omega\left(\zeta_{0}^{\prime}, 0\right) \subset R^{n+2}$ of the point $\left(\zeta_{0}^{\prime}, r=0\right)$. Often we shall restrict ourselves to a subset $\Omega_{+}\left(\zeta_{0}^{\prime}, 0\right) \subset \Omega\left(\zeta_{0}^{\prime}, 0\right)$ which consists of the points $\left(\zeta^{\prime}, r\right)$ with $\eta^{\prime} \geqslant 0$ and $r \geqslant 0$. Let $\kappa_{1}^{\prime}, \kappa_{2}^{\prime}, \ldots, \kappa_{m}^{\prime}$ be all the different eigenvalues of the matrix $M^{\prime}\left(\zeta_{0}^{\prime}, 0\right)$ with multiplicities $q_{1}, q_{2}, \ldots, q_{m}$. There exists an analytic matrix-function

$$
X^{\prime}\left(\zeta^{\prime}, r\right)=\left(X_{1}^{\prime}, X_{2}^{\prime}, \ldots, X_{m}^{\prime}\right)
$$

such that

$$
\left(X^{\prime}\right)^{-1} M^{\prime} X^{\prime}=\operatorname{diag}\left(M_{1}^{\prime}, M_{2}^{\prime}, \ldots, M_{m}^{\prime}\right)
$$

and the matrix $M_{j}^{\prime}\left(\zeta_{0}^{\prime}, 0\right), j=1,2, \ldots, m$, has the only eigenvalue $\kappa_{j}^{\prime}$. If $\operatorname{Re} \kappa_{j}^{\prime}>0$ or $\operatorname{Re} \kappa_{j}^{\prime}<0$, we may even assume that correspondingly

$$
\begin{array}{ll}
\text { (a) } & \operatorname{Re} M_{j}^{\prime}\left(\zeta^{\prime}, r\right) \geqslant \delta I \\
& \text { or } \\
\text { (b) } & \operatorname{Re} M_{j}^{\prime}\left(\zeta^{\prime}, r\right) \leqslant-\delta I
\end{array}
$$

for any $\left(\zeta^{\prime}, r\right) \in \Omega\left(\zeta_{0}^{\prime}, 0\right)$.

Now consider the case $\operatorname{Re} \kappa_{j}^{\prime}=0$. Due to the strict hyperbolicity of system (1.1) this situation may occur only if $\eta_{0}^{\prime}=0$. Moreover, the eigenspace of the corresponding matrix $M_{j}^{\prime}\left(\zeta_{0}^{\prime}, 0\right)$ will be one-dimensional. If $\left(\xi_{-}^{\prime}\right)_{0}=0$ we get from (3.29) that $M^{\prime}\left(\zeta_{0}^{\prime}, 0\right)$ is a diagonal matrix with distinct eigenvalues, and thus $M_{j}^{\prime}\left(\zeta^{\prime}, r\right)$ is a scalar function. We shall see later in the proof of Lemma 3.1 that for such scalar blocks the well-posedness of the Cauchy problem implies

$$
\begin{array}{ll}
\text { (a) } & \left(I+r M_{j}^{\prime}\left(\zeta^{\prime}, r\right)\right) *\left(I+r M_{j}^{\prime}\left(\zeta^{\prime}, r\right)\right)-I \geqslant \delta \eta I \\
& \text { or } \\
\text { (b) }\left(I+r M_{j}^{\prime}\left(\zeta^{\prime}, r\right)\right) *\left(I+r M_{j}^{\prime}\left(\zeta^{\prime}, r\right)\right)-I \leqslant-\delta \eta I
\end{array}
$$

for all $\left(\zeta^{\prime}, r\right) \in \Omega_{+}\left(\zeta_{0}^{\prime}, 0\right)$. Thus we restrict ourselves to the more difficult case $\left(\xi_{-}^{\prime}\right)_{0} \neq 0$ in which, however, one can benefit from the presence of dissipation. 
The matrix $M_{j}^{\prime}\left(\zeta_{0}^{\prime}, 0\right)$ may be thought of as a Jordan cell of the order $q_{j}$. Following Gustafsson et al. [5] we consider a matrix

$$
\hat{M}_{j}^{\prime}\left(\zeta^{\prime}, r\right)=(i r)^{-1} \ln \left(I+r M_{j}^{\prime}\left(\zeta^{\prime}, r\right)\right) .
$$

Obviously, the matrix $\hat{M}_{j}^{\prime}\left(\zeta^{\prime}, r\right)$ is analytic in $\Omega\left(\zeta_{0}^{\prime}, 0\right)$ and $\hat{M}_{j}^{\prime}\left(\zeta_{0}^{\prime}, 0\right)=-i M_{j}^{\prime}\left(\zeta_{0}^{\prime}, 0\right)$ is a Jordan cell with the real eigenvalue $\lambda_{j}^{\prime}=-i \kappa_{j}^{\prime}$. The matrix $X_{j}^{\prime}\left(\zeta^{\prime}, r\right)$ in (3.30) may be chosen in such a way that $\hat{M}_{j}^{\prime}\left(\zeta^{\prime}, r\right)$ has a form

$$
\hat{M}_{j}^{\prime}\left(\zeta^{\prime}, r\right)=\lambda_{j}^{\prime} I+\left(\begin{array}{ccccc}
e_{q_{j}-1} & 1 & 0 & \cdots & 0 \\
e_{q_{j}-2} & 0 & 1 & & 0 \\
\vdots & & \ddots & \vdots \\
e_{0} & 0 & \cdots & & 0
\end{array}\right),
$$

where $e_{q}=e_{q}\left(\zeta^{\prime}, r\right), q=0,1, \ldots, q_{j}-1$, depend analytically on $\zeta^{\prime}, r$ and vanish at the point $\left(\zeta_{0}^{\prime}, 0\right)$. Let $\left(\zeta^{\prime}, r\right) \in \Omega_{+}\left(\zeta_{0}^{\prime}, 0\right)$ with $r>0$. Denote by $\rho_{j}$ the number of eigenvalues $\kappa$ of the matrix $I+r M_{j}^{\prime}\left(\zeta^{\prime}, r\right)$ with $|\kappa|<1$. Since for $\left(\zeta^{\prime}, r\right)$ as above the characteristic equation (3.16) has no solutions with $|\kappa|=1$, it follows that the number $\rho_{j}$ is independent of $\left(\zeta^{\prime}, r\right)$. It is easy to show that the mapping $\kappa \rightarrow \lambda^{\prime}=$ $(i r)^{-1} \ln \kappa$ provides a one-to-one correspondence between the eigenvalues of the matrices $I+r M_{j}^{\prime}\left(\zeta^{\prime}, r\right)$ and $\hat{M}_{j}^{\prime}\left(\zeta^{\prime}, r\right)$ such that the eigenvalues $\kappa$ with $|\kappa|<1$ are mapped into the eigenvalues $\lambda^{\prime}$ with $\operatorname{Im} \lambda^{\prime}>0$. Therefore the matrix $\hat{M}_{j}^{\prime}\left(\zeta^{\prime}, r\right)$ has $\rho_{j}$ eigenvalues with $\operatorname{Im} \lambda^{\prime}>0$ and $q_{j}-\rho_{j}$ with $\operatorname{Im} \lambda^{\prime}<0$. Let us partition the matrix $X_{j}^{\prime}\left(\zeta^{\prime}, r\right)$ as

$$
X_{j}^{\prime}=\left(X_{\mathrm{I}, j}^{\prime}, X_{\mathrm{II}, j}^{\prime}\right),
$$

where the matrix $X_{\mathrm{I}, j}^{\prime}$ consists of the first $\rho_{j}$ columns of $X_{j}^{\prime}$ and $X_{\mathrm{II}, j}^{\prime}$ of the remaining $q_{j}-\rho_{j}$ columns. If $v_{j}^{\prime}$ is a $q_{j}$-dimensional column-vector, we shall similarly partition it as

$$
v_{j}^{\prime}=\left(\begin{array}{c}
v_{\mathrm{I}, j}^{\prime} \\
v_{\mathrm{II}, j}^{\prime}
\end{array}\right) .
$$

As in [7, Lemma 2.6] one can show that there exists a matrix-function $U_{j}\left(\zeta^{\prime}, r\right)$ defined for $\left(\zeta^{\prime}, r\right) \in \Omega\left(\zeta_{0}^{\prime}, 0\right)$ and continuous at the point $\left(\zeta_{0}^{\prime}, 0\right)$ such that $U_{j}\left(\zeta_{0}^{\prime}, 0\right)$ $=I$ and

$$
\begin{aligned}
\left(U_{j}\left(\zeta^{\prime}, r\right)\right)^{-1}\left(I+r M_{j}^{\prime}\left(\zeta^{\prime}, r\right)\right) U_{j}\left(\zeta^{\prime}, r\right) & \\
& =\left(\begin{array}{ccccc}
\kappa_{j 1} & \gamma & 0 & \ldots & 0 \\
0 & \kappa_{j 2} & \gamma & \cdots & 0 \\
\vdots & & . & & \vdots \\
0 & \ldots & & & \kappa_{j q_{j}}
\end{array}\right)=\left(\begin{array}{cc}
N_{j 11} & N_{j 12} \\
0 & N_{j 22}
\end{array}\right) .
\end{aligned}
$$

Here $\gamma=i r$, and for $\left(\zeta^{\prime}, r\right) \in \Omega_{+}\left(\zeta_{0}^{\prime}, 0\right), r>0$, the eigenvalues with $|\kappa|<1$ stand in the first $\rho_{j}$ rows, so that the spectra of the matrices $N_{j 11}$ and $N_{j 22}$ lie correspondingly inside and outside the unit circle $|\kappa|=1$. 
In order to build a full symmetrizer for the matrix $M_{1}(\zeta)$ in a neighborhood of the point $\zeta=0$, we shall first construct a symmetrizer for the matrix $I+r M_{j}^{\prime}\left(\zeta^{\prime}, r\right)$ in the case $\operatorname{Re} \kappa_{j}^{\prime}=0$. The main result of this subsection is

THEOREM 3.1. There exists a Hermitian matrix-function $R_{j}^{\prime}\left(\zeta^{\prime}, r\right)$ depending smoothly on $\left(\zeta^{\prime}, r\right) \in \Omega\left(\zeta_{0}^{\prime}, 0\right)$ and satisfying

$$
\begin{aligned}
\left(v_{j}^{\prime}\right) * R_{j}^{\prime}\left(\zeta^{\prime}, r\right) v_{j}^{\prime} \geqslant-c\left|v_{\mathrm{I}, j}^{\prime}\right|^{2}+\left|v_{\mathrm{II}, j}^{\prime}\right|^{2} \quad \text { for any }\left(\zeta^{\prime}, r\right) \in \Omega\left(\zeta_{0}^{\prime}, 0\right), \\
\left(I+r M^{\prime}\left(\zeta^{\prime}, r\right)\right)^{*} R^{\prime}\left(\zeta^{\prime}, r\right)\left(I+r M^{\prime}\left(\zeta^{\prime}, r\right)\right)-R^{\prime}\left(\zeta^{\prime}, r\right) \geqslant \delta \eta I \\
\quad f o r \text { any }\left(\zeta^{\prime}, r\right) \in \Omega_{+}\left(\zeta_{0}^{\prime}, 0\right),
\end{aligned}
$$

where $\delta$ and $c$ are positive constants and $c$ may be chosen arbitrarily small.

We shall use the construction of Kreiss in [7] in order to build the above symmetrizer for the matrix $i \hat{M}_{j}^{\prime}\left(\zeta^{\prime}, r\right)$, so that in addition to (3.39) the estimate

$$
\operatorname{Re}\left(i R_{j}^{\prime}\left(\zeta^{\prime}, r\right) \hat{M}_{j}^{\prime}\left(\zeta^{\prime}, r\right)\right) \geqslant \delta \eta^{\prime} I
$$

holds for any $\left(\zeta^{\prime}, r\right) \in \Omega_{+}\left(\zeta_{0}^{\prime}, 0\right)$. Then as in [5] one obtains for the matrix $I+r M_{j}^{\prime}\left(\zeta^{\prime}, r\right)=\exp \left(i r \hat{M}_{j}^{\prime}\left(\zeta^{\prime}, r\right)\right)$ estimate (3.40). Unfortunately, the matrix $i \hat{M}_{j}^{\prime}\left(\zeta^{\prime}, r\right)$ does not satisfy the double-sided resolvent condition of $[5$, p. 685] and, as a result, the coefficients $e_{q}\left(\zeta^{\prime}, r\right)$ in (3.35) may be complex for $\eta=0$. The following lemma provides, however, the necessary estimates for the imaginary part of $e_{q}\left(\zeta^{\prime}, r\right)$.

LEMMA 3.1. There is a neighborhood $\Omega_{+}\left(\zeta_{0}^{\prime}, 0\right)$ and positive constants $K$ and $\delta$ such that the estimates

$$
\begin{gathered}
\left|\operatorname{Im} e_{q}\left(\zeta^{\prime}, r\right)\right| \leqslant K\left|\operatorname{Im} e_{0}\left(\zeta^{\prime}, r\right)\right|, \quad q=1,2, \ldots, q_{j}-1, \\
\left|\operatorname{Im} e_{0}\left(\zeta^{\prime}, r\right)\right| \geqslant \delta\left(\eta^{\prime}+r^{2 m-1}\right) \geqslant \delta \eta^{\prime},
\end{gathered}
$$

hold for any $\left(\zeta^{\prime}, r\right) \in \Omega_{+}\left(\zeta_{0}^{\prime}, 0\right)$. Here $m$ is one of the integers $m_{j}$ appearing in (1.28).

Proof. Consider the characteristic equation

$$
\begin{aligned}
\operatorname{det}\left(\lambda^{\prime} I\right. & \left.-\hat{M}_{j}^{\prime}\left(\zeta^{\prime}, r\right)\right) \\
& =\left(\lambda^{\prime}-\lambda_{j}^{\prime}\right)^{q_{j}}-e_{q_{j}-1}\left(\zeta^{\prime}, r\right)\left(\lambda^{\prime}-\lambda_{j}^{\prime}\right)^{q_{j}-1}-\cdots-e_{0}\left(\zeta^{\prime}, r\right)=0
\end{aligned}
$$

in a neighborhood $\left(\lambda^{\prime}, \zeta^{\prime}, r\right) \in \Omega\left(\lambda_{j}^{\prime}\right) \times \Omega\left(\zeta_{0}^{\prime}, 0\right)$, where $\Omega\left(\lambda_{j}^{\prime}\right)$ is a small neighborhood of $\lambda_{j}^{\prime}$ in $\mathbf{C}$. We shall show that this equation is equivalent to an equation

$$
s^{\prime}-g_{i}\left(\lambda^{\prime}, \xi_{-}^{\prime}, r\right)=0
$$

where $g_{i}$ is one of the functions $g_{j}, j=1,2, \ldots, k$, defined in (1.26) with $\xi^{\prime}$ replaced by $\left(\lambda^{\prime}, \xi_{-}^{\prime}\right)$, and the value of the index $i$ is uniquely determined by the equality

$$
s_{0}^{\prime}=g_{i}\left(\lambda_{j}^{\prime},\left(\xi_{-}^{\prime}\right)_{0}, 0\right) \text {. }
$$

Indeed, introduce a mapping

$$
\kappa^{\prime}=\varphi\left(\lambda^{\prime}, r\right)=\left(\exp \left(i \lambda^{\prime} r\right)-1\right) / r
$$

The function $\varphi\left(\lambda^{\prime}, r\right)$ depends analytically on $\lambda^{\prime}$ and $r$ and the mapping $\lambda^{\prime} \rightarrow \varphi\left(\lambda^{\prime}, r\right)$ is one-to-one for bounded $\lambda^{\prime}$ and small $r$. Since $M_{j}^{\prime}\left(\zeta^{\prime}, r\right)=\varphi\left(\hat{M}_{j}^{\prime}\left(\zeta^{\prime}, r\right), r\right)$, the mapping in (3.47) transforms the roots of Eq. (3.44) into the roots of the equation

$$
\operatorname{det}\left(\kappa^{\prime} I-M_{j}^{\prime}\left(\zeta^{\prime}, r\right)\right)=0 \text {. }
$$


Denote $\hat{L}\left(\lambda^{\prime}, \zeta^{\prime}, r\right)=L^{\prime}\left(\varphi\left(\lambda^{\prime}, r\right), \zeta^{\prime}, r\right)$. The mapping in (3.47) provides a one-to-one correspondence between the roots of the equations

$$
\operatorname{det} \hat{L}^{\prime}\left(\lambda^{\prime}, \zeta^{\prime}, r\right)=0
$$

and

$$
\operatorname{det} L^{\prime}\left(\kappa^{\prime}, \zeta^{\prime}, r\right)=0 \text {. }
$$

Formula (3.28) implies that Eqs. (3.48) and (3.50) are equivalent for $\boldsymbol{\kappa}^{\prime}$ in a small complex neighborhood $\Omega\left(\kappa_{j}^{\prime}\right)$ of $\kappa_{j}^{\prime}$. Therefore Eqs. (3.44) and (3.49) are equivalent for $\lambda^{\prime}$ in a corresponding neighborhood $\Omega\left(\lambda_{j}^{\prime}\right)$. Note that the matrix-function $\hat{L}^{\prime}\left(\lambda^{\prime}, \zeta^{\prime}, r\right)$ coincides with the matrix-function $L^{\prime}\left(x, t, \xi^{\prime}, s^{\prime}, r\right)$ defined in (1.24), provided $x, t$ are frozen at a boundary point and $\xi^{\prime}$ is replaced by the pair $\left(\lambda^{\prime}, \xi_{-}^{\prime}\right)$. The functions $g_{j}$ in (1.26) are analytically extensible to a complex neighborhood of the real vector $\left(\lambda_{j}^{\prime},\left(\xi_{-}^{\prime}\right)_{0}\right)$. Thus Eq. (3.49) is equivalent to

$$
\prod_{i=1}^{k}\left(s^{\prime}-g_{i}\left(\lambda^{\prime}, \xi_{-}^{\prime}, r\right)\right)=0 \text {. }
$$

In the above product only one term vanishes at the point $s^{\prime}=s_{0}^{\prime}$ and $\left(\lambda^{\prime}, \xi_{-}^{\prime}, r\right)=$ $\left(\lambda_{j}^{\prime},\left(\xi_{-}^{\prime}\right)_{0}, 0\right)$ thus determining the index $i$ in (3.45). We shall omit this index and rewrite Eq. (3.45) as

$$
f\left(\lambda^{\prime}, \zeta^{\prime}, r\right)=i\left(s^{\prime}-g\left(\lambda^{\prime}, \xi_{-}^{\prime}, r\right)\right)=0 .
$$

If the multiplicity $q_{j}$ of the root $\lambda_{j}^{\prime}$ is one, for any $\left(\zeta^{\prime}, r\right) \in \Omega_{+}\left(\zeta^{\prime}, 0\right)$ the root $\lambda^{\prime}$ of (3.52) satisfies the estimate

$$
\begin{aligned}
\left|\operatorname{Im} \lambda^{\prime}\right| & \geqslant \delta\left|s^{\prime}-g\left(\operatorname{Re} \lambda^{\prime}, \xi_{-}^{\prime}, r\right)\right| \\
& \geqslant \delta\left(\eta^{\prime}-\operatorname{Re} g\left(\operatorname{Re} \lambda^{\prime}, \xi_{-}^{\prime}, r\right)\right) \geqslant \delta \eta^{\prime},
\end{aligned}
$$

since $\operatorname{Re} g\left(\operatorname{Re} \lambda^{\prime}, \xi_{-}^{\prime}, r\right) \leqslant 0$ (the last is a necessary condition for stability of the Cauchy problem). Since

$$
\operatorname{Re}\left(i \hat{M}_{j}^{\prime}\left(\zeta^{\prime}, r\right)\right)=-\operatorname{Im} e_{0}\left(\zeta^{\prime}, r\right)=-\operatorname{Im} \lambda^{\prime},
$$

in accordance with the sign of $\operatorname{Im} \lambda^{\prime}$ we arrive at one of the estimates in (3.33). In case $q_{j}>1$ we consider the difference

$$
f\left(\lambda^{\prime}, \zeta^{\prime}, r\right)-\overline{f\left(\overline{\lambda^{\prime}}, \zeta^{\prime}, r\right)}=i\left(2 \eta^{\prime}-h\left(\lambda^{\prime}, \xi_{-}^{\prime}, r\right)\right),
$$

where

$$
h\left(\lambda^{\prime}, \xi_{-}^{\prime}, r\right)=g\left(\lambda^{\prime}, \xi_{-}^{\prime}, r\right)+\overline{g\left(\overline{\lambda^{\prime}}, \xi_{-}^{\prime}, r\right)}
$$

and - is a symbol of complex conjugation. For $r \geqslant 0$ and real $\lambda^{\prime}$ the function $h\left(\lambda^{\prime}, \xi_{-}, r\right)$, according to Assumption 1.4, satisfies the estimate

$$
-\delta_{1} r^{2 m-1} \leqslant h\left(\lambda^{\prime}, \xi_{-}^{\prime}, r\right) \leqslant-\delta_{2} r^{2 m-1},
$$

where $m=m_{i}$ with the index $i$ determined by (3.46). Note that $h\left(\lambda^{\prime}, \xi_{-}^{\prime}, r\right)$ depends analytically on the complex $\lambda^{\prime}$ and real $\xi_{-}^{\prime}$ and $r$. Expanding $h\left(\lambda^{\prime}, \xi_{-}^{\prime}, r\right)$ in a power series according to $r$,

$$
h\left(\lambda^{\prime}, \xi_{-}^{\prime}, r\right)=\sum_{i=0}^{\infty} h_{i}\left(\lambda^{\prime}, \xi_{-}^{\prime}\right) r^{i}
$$


we derive from (3.56) that the first $2 m-1$ coefficients $h_{i}\left(\lambda^{\prime}, \xi_{-}^{\prime}\right)$ vanish for real $\lambda^{\prime}$ and, hence, for all complex $\lambda^{\prime} \in \Omega\left(\lambda_{j}^{\prime}\right)$. Thus we have for all $\lambda^{\prime} \in \Omega\left(\lambda_{j}^{\prime}\right)$ and $\left(\zeta^{\prime}, r\right) \in \Omega_{+}\left(\zeta_{0}^{\prime}, 0\right)$

$$
\left|f\left(\lambda^{\prime}, \zeta^{\prime}, r\right)-\overline{f\left(\overline{\lambda^{\prime}}, \zeta^{\prime}, r\right)}\right| \leqslant K\left(\eta^{\prime}+r^{2 m-1}\right)
$$

and

$$
\left|\frac{\partial}{\partial \lambda^{\prime}}\left[f\left(\lambda^{\prime}, \zeta^{\prime}, r\right)-\overline{f\left(\overline{\lambda^{\prime}}, \zeta^{\prime}, r\right)}\right]\right|=\left|\frac{\partial}{\partial \lambda^{\prime}} h\left(\lambda^{\prime}, \xi_{-}^{\prime}, r\right)\right| \leqslant K r^{2 m-1} .
$$

Denote $f_{0}\left(\zeta^{\prime}, r\right)=f\left(\lambda_{j}^{\prime}, \zeta^{\prime}, r\right)$. Then

$$
\operatorname{Im} f_{0}\left(\zeta^{\prime}, r\right)=\eta^{\prime}-\frac{1}{2} h\left(\lambda_{j}^{\prime}, \xi_{-}^{\prime}, r\right) \geqslant \eta^{\prime}+\delta r^{2 m-1} .
$$

Therefore one can replace the right-hand sides of estimates (3.57) and (3.58) by $K\left|\operatorname{Im} f_{0}\left(\zeta^{\prime}, r\right)\right|$. Thus the function $f\left(\lambda^{\prime}, \zeta^{\prime}, r\right)$ satisfies the conditions of Lemma 3.2 proven below. The relation with the notations of the lemma is as follows:

$$
z=\lambda^{\prime}-\lambda_{j}^{\prime}, \quad w=\left(\zeta^{\prime}-\zeta_{0}^{\prime}, r\right), \mathscr{D}=\left\{w \mid\left(\zeta^{\prime}, r\right) \in \Omega_{+}\left(\zeta_{0}^{\prime}, 0\right)\right\}
$$

Since the functions $-e_{q}\left(\zeta^{\prime}, r\right), q=0,1, \ldots, q_{j}-1$, are the coefficients of the Weierstrass polynomial corresponding to $f\left(\lambda^{\prime}, \zeta^{\prime}, r\right)$, we arrive, according to Lemma 3.2 and estimate (3.59), at the required estimates (3.42) and (3.43).

Let $\left(\zeta^{\prime}, r\right) \in \Omega_{+}\left(\zeta_{0}^{\prime}, 0\right)$ with $\eta^{\prime}>0$. Then, as follows from estimate (3.43), $\operatorname{Im} e_{0}\left(\zeta^{\prime}, r\right)$ is of constant sign. As in [7, Lemma 2.7] we shall show that the number $\rho_{j}$ of the eigenvalues of $\hat{M}_{j}^{\prime}\left(\zeta^{\prime}, r\right)$ in the half-plane $\operatorname{Im} \lambda^{\prime}>0$ is given by the formula

$$
\rho_{j}= \begin{cases}q_{j} / 2 & \text { if } q_{j} \text { is even } \\ \left(q_{j}-1\right) / 2 & \text { if } q_{j} \text { is odd and } \operatorname{Im} e_{0}\left(\zeta^{\prime}, r\right)>0 \\ \left(q_{j}+1\right) / 2 & \text { if } q_{j} \text { is odd and } \operatorname{Im} e_{0}\left(\zeta^{\prime}, r\right)<0\end{cases}
$$

Indeed, since this number is independent of $\left(\zeta^{\prime}, r\right)$ as above, we shall set $r=0$, $\xi^{\prime}=\left(\xi^{\prime}\right)_{0}$ and let $\eta^{\prime}>0$. Then $\left|\operatorname{Im} e_{0}\left(\zeta^{\prime}, 0\right)\right| \geqslant \delta \eta^{\prime}$ and $e_{q}\left(\zeta^{\prime}, 0\right)=O\left(\eta^{\prime}\right), q=$ $0,1, \ldots, q_{j}-1$. Therefore the eigenvalues $\lambda^{\prime}$ of the matrix $\hat{M}_{j}^{\prime}\left(\zeta^{\prime}, 0\right)$ may be written in the form

$$
\lambda^{\prime}=\lambda_{j}^{\prime}+e_{0}\left(\zeta^{\prime}, 0\right)^{1 / q_{j}} \cdot\left(1+O\left(\eta^{\prime}\right)^{1 / q_{j}}\right),
$$

and formula (3.60) follows easily.

Using estimates (3.42) and (3.43) and formula (3.60) we are able to construct the required symmetrizer $R_{j}^{\prime}\left(\zeta^{\prime}, r\right)$ for the matrix $i \hat{M}_{j}^{\prime}\left(\zeta^{\prime}, r\right)$. In the notations of [7] the matrix $i \hat{M}_{j}^{\prime}\left(\zeta^{\prime}, r\right)$ is represented as

$$
i \hat{M}_{j}^{\prime}\left(\zeta^{\prime}, r\right)=i \lambda_{j}^{\prime} I+i C+i E\left(\zeta^{\prime}, r\right)+N\left(\zeta^{\prime}, r\right)
$$

where

$$
C=\left(\begin{array}{cccc}
0 & 1 & \cdots & 0 \\
0 & 0 & 1 & 0 \\
\vdots & & \ddots & \vdots \\
0 & & \cdots & 0
\end{array}\right), \quad E\left(\zeta^{\prime}, r\right)=\left(\begin{array}{cccc}
\operatorname{Re} e_{q_{j}-1} & 0 & \ldots & 0 \\
\vdots & & & \vdots \\
\operatorname{Re} e_{0} & 0 & \ldots & 0
\end{array}\right)
$$




$$
N\left(\zeta^{\prime}, r\right)=-\left(\begin{array}{cccc}
\operatorname{Im} e_{q_{j}-1} & 0 & \cdots & 0 \\
\vdots & & & \vdots \\
\operatorname{Im} e_{0} & 0 & \cdots & 0
\end{array}\right) .
$$

Then $R^{\prime}\left(\zeta^{\prime}, r\right)=(D+B)-i \eta^{\prime} F$, where $D, B$ and $F$ are correspondingly the matrices $D, \varepsilon B$ and $F$ defined in [7, Lemmas 4.1, 4.3 and 4.4].

Thus Theorem 3.1 is proved modulo the following general

LEMMA 3.2. Let $f(z, w)$ be a function of a complex variable $z$ and a real vector variable $w \in R^{n}$, which depends continuously on $(z, w)$ and analytically on $z$ for $z$ and $w$ in some neighborhood $\Omega_{z} \times \Omega_{w}$ of the point $z=0, w=0$. Let $z=0$ be a null of the function $f(z, 0)$ of multiplicity $q$. Denote by

$$
p(z, w)=\sum_{k=0}^{q-1} e_{k}(w) z^{k}+z^{q}
$$

the Weierstrass polynomial corresponding to the function $f(z, w)$. Suppose that for any $w$ belonging to some set $\mathscr{D} \subset R^{n}$ and any $z$ in a neighborhood of zero the following estimates hold:

$$
\begin{gathered}
|\bar{f}(z, w)-f(z, w)| \leqslant K\left|\operatorname{Im} f_{0}(w)\right|, \\
\left|\frac{\partial \bar{f}(z, w)}{\partial z}-\frac{\partial f(z, w)}{\partial z}\right| \leqslant K\left|\operatorname{Im} f_{0}(w)\right|,
\end{gathered}
$$

where $\bar{f}(z, w)=\overline{f(\bar{z}, w)}$ and $f_{0}(w)=f(0, w)$. Then there is a neighborhood $\Omega_{w}^{\prime} \subset R^{n}$ of the point $w=0$ and positive constants $K_{1}$ and $\delta$ such that for any $w \in \mathscr{D} \cap \Omega_{w}^{\prime}$ the estimates

$$
\left|\operatorname{Im} e_{k}(w)\right| \leqslant K_{1}\left|\operatorname{Im} f_{0}(w)\right|, \quad k=0,1, \ldots, q-1,
$$

and

$$
\left|\operatorname{Im} e_{0}(w)\right| \geqslant \delta\left|\operatorname{Im} f_{0}(w)\right|
$$

hold.

Proof. Let $\Gamma=\{z \in \mathrm{C}|| z \mid=\varepsilon\} \subset \Omega_{z}$ be a positive oriented circle and $\Omega_{w}^{\prime} \subset \Omega_{w}$ a neighborhood of zero, such that $f(z, 0) \neq 0$ for $0<|z| \leqslant \varepsilon$ and $f(z, w) \neq 0$ for any $(z, w) \in \Gamma \times \Omega_{w}^{\prime}$. Let us fix $w \in \mathscr{D} \cap \Omega_{w}^{\prime}$. Denote by $z_{1}, z_{2}, \ldots, z_{q}$ the roots of the equation $f(z, w)=0$ and consider their symmetric functions $\sigma_{j}(w)=z_{1}^{j}+z_{2}^{j}$ $+\cdots+z_{q}^{j}, j=1,2, \ldots, q$. By the Cauchy integral formula

$$
\sigma_{j}(w)=\frac{1}{2 \pi i} \oint_{\Gamma} \frac{z^{j} f^{\prime}(z, w)}{f(z, w)} d z
$$

where $f^{\prime}(z, w)=\partial f(z, w) / \partial z$. Therefore

$$
\overline{\boldsymbol{\sigma}}_{j}(w)=\bar{z}_{1}^{j}+\bar{z}_{2}^{j}+\cdots+\bar{z}_{q}^{j}=\frac{1}{2 \pi i} \oint_{\Gamma} \frac{z^{j} \bar{f}^{\prime}(z, w)}{\bar{f}(z, w)} d z
$$

and

$$
\operatorname{Im} \sigma_{j}(w)=-\frac{1}{4 \pi} \oint z^{j}\left(\frac{f^{\prime}}{f}-\frac{\bar{f}^{\prime}}{\bar{f}}\right) d z=-\frac{1}{4 \pi} \oint z^{j} \frac{f^{\prime}(\bar{f}-f)+f\left(f^{\prime}-\bar{f}^{\prime}\right)}{f \cdot \bar{f}} d z .
$$


Since $f(z, w) \cdot \bar{f}(z, w) \neq 0$ for any $(z, w) \in \Gamma \times \Omega_{w}^{\prime}$, we get from (3.61) and (3.62) an estimate

$$
\left|\operatorname{Im} \sigma_{j}(w)\right| \leqslant K\left|\operatorname{Im} f_{0}(w)\right|, \quad j=1,2, \ldots, q,
$$

where the constant $K$ is independent of $w \in \Omega_{w}^{\prime}$. Since $e_{k}(w)$ is a polynomial of $\sigma_{j}(w), j=1,2, \ldots, q$, with real coefficients, we obtain for $\operatorname{Im} e_{k}(w)$ the required estimate (3.63). The coefficient $e_{0}(w)$ is given by

$$
e_{0}(w)=(-1)^{q} z_{1} z_{2} \cdots z_{q}=-\frac{1}{q} \sigma_{q}(w)+s\left(\sigma_{1}, \sigma_{2}, \ldots, \sigma_{q-1}\right)
$$

where $s$ is a polynomial with real coefficients. It is clear from the consideration of degree that the polynomial $s$ has no linear terms. Since $\sigma_{j}(0)=0$, it follows that $\left|\operatorname{Im} s\left(\sigma_{1}, \sigma_{2}, \ldots, \sigma_{q-1}\right)\right| \leqslant \delta_{1}\left|\operatorname{Im} f_{0}(w)\right|$, where $\delta_{1}$ is arbitrarily small if one selects a small neighborhood $\Omega_{w}^{\prime}$. Let us rewrite the integral expression for $\operatorname{Im} \sigma_{q}(w)$ :

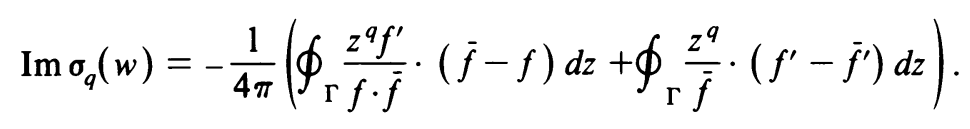

The functions $z^{q} f^{\prime}(z, w) / f(z, w) \bar{f}(z, w)$ and $z^{q} / \bar{f}(z, w)$ are continuous in $\Gamma \times \Omega_{w}^{\prime}$ and, hence, the difference of their values at the points $(z, w)$ and $(z, 0)$ may be bounded by an arbitrarily small constant if one chooses a sufficiently small neighborhood $\Omega_{w}^{\prime}$. Note that $z^{q} / \bar{f}(z, 0)$ is an analytic function of $z$, and therefore

$$
\oint_{\Gamma} \frac{z^{q}}{\bar{f}(z, 0)}\left(f^{\prime}(z, w)-\bar{f}^{\prime}(z, w)\right) d z=0
$$

Similarly,

$$
\frac{z^{q} \cdot f^{\prime}(z, 0)}{f(z, 0) \bar{f}(z, 0)}=\frac{q}{\overline{f_{q}}(0,0)} \cdot \frac{1}{z}+g(z)
$$

where $\bar{f}_{q}(0,0)=(q !)^{-1} \partial^{q} \bar{f}(z, 0) /\left.\partial z^{q}\right|_{z=0}$ is nonzero and $g(z)$ is an analytic function. Then

$$
-\frac{1}{4 \pi} \oint_{\Gamma} \frac{z^{q} f^{\prime}(z, 0)}{f(z, 0) \bar{f}(z, 0)} \cdot(\bar{f}(z, w)-f(z, w)) d z=\frac{-q \cdot \operatorname{Im} f_{0}(w)}{\bar{f}_{q}(0,0)} .
$$

Now, using estimates (3.61) and (3.62), we obtain for sufficiently small $\Omega_{w}^{\prime}$

$$
\left|\operatorname{Im} \sigma_{q}(w)\right| \geqslant \delta\left|\operatorname{Im} f_{0}(w)\right|
$$

and therefore

$$
\left|\operatorname{Im} e_{0}(w)\right| \geqslant \delta\left|\operatorname{Im} f_{0}(w)\right|
$$

for some constant $\delta$ independent of $w \in \mathscr{Q} \cap \Omega_{w}^{\prime}$. Q.E.D.

Having constructed the symmetrizers $R_{j}^{\prime}\left(\zeta^{\prime}, z\right)$ in the case $\operatorname{Re} \kappa_{j}^{\prime}=0$ and $q_{j}>1$, we define the matrix-functions $R_{j}^{\prime}\left(\zeta^{\prime}, z\right)$ for the remaining indices as follows:

$$
\begin{array}{lll}
\text { (a) } & R_{j}^{\prime}\left(\zeta^{\prime}, r\right)=I_{q_{j}} & \text { if }(3.32 \mathrm{a}) \text { or }(3.33 \mathrm{a}) \text { hold } \\
\text { (b) } & R_{j}^{\prime}\left(\zeta^{\prime}, r\right)=-c I_{q_{j}} & \text { if }(3.32 \mathrm{~b}) \text { or }(3.33 \mathrm{~b}) \text { hold }
\end{array}
$$


Here $c$ is a small positive constant and will be specified later. Finally we define the symmetrizer for the matrix $M_{1}(\zeta)$ in the neighborhood $\Omega\left(\zeta_{0}^{\prime}, 0\right)$ as

$$
\begin{aligned}
R^{\prime}\left(\zeta^{\prime}, r\right)= & {\left[\left(X^{\prime}\left(\zeta^{\prime}, r\right)\right)^{-1}\right] * \cdot \operatorname{diag}\left(R_{1}^{\prime}\left(\zeta^{\prime}, r\right), R_{2}^{\prime}\left(\zeta^{\prime}, r\right), \ldots, R_{m}^{\prime}\left(\zeta^{\prime}, r\right)\right) } \\
& \cdot\left(X^{\prime}\left(\zeta^{\prime}, r\right)\right)^{-1} .
\end{aligned}
$$

The matrix $R^{\prime}\left(\zeta^{\prime}, r\right)$ is Hermitian and satisfies for $\left(\zeta^{\prime}, r\right) \in \Omega_{+}\left(\zeta^{\prime}, r\right)$ the estimate

$$
M_{1}^{*}(\zeta) R^{\prime}\left(\zeta^{\prime}, r\right) M_{1}(\zeta)-R^{\prime}\left(\zeta^{\prime}, r\right) \geqslant \delta \eta I
$$

Let $v^{\prime}$ be a $k$-dimensional column vector. We shall partition it as

$$
v^{\prime}=\left(\begin{array}{c}
v_{\mathrm{I}}^{\prime} \\
v_{\mathrm{II}}^{\prime}
\end{array}\right)
$$

where $v_{\mathrm{I}}^{\prime}$ includes those components of $v^{\prime}$ which correspond to the blocks $M_{j}^{\prime}$ in (3.32b) and (3.33b) as well as the components $v_{\mathrm{I}, j}^{\prime}$ defined in (3.37), and $v_{\mathrm{II}}^{\prime}$ consists of the remaining components of $v^{\prime}$. In the same way we partition the columns of the matrix $X^{\prime}\left(\zeta^{\prime}, r\right)$

$$
X^{\prime}\left(\zeta^{\prime}, r\right)=\left(X_{\mathrm{I}}^{\prime}\left(\zeta^{\prime}, r\right), X_{\mathrm{II}}^{\prime}\left(\zeta^{\prime}, r\right)\right)
$$

Due to estimate (3.39) and definition (3.66) we have

$$
\left(v^{\prime}\right)^{*}\left(X^{\prime}\left(\zeta^{\prime}, r\right)\right)^{*} R^{\prime}\left(\zeta^{\prime}, r\right) X^{\prime}\left(\zeta^{\prime}, r\right) v^{\prime} \geqslant\left|v_{\mathrm{II}}^{\prime}\right|^{2}-c\left|v_{\mathrm{I}}^{\prime}\right|^{2}
$$

In order to define the symmetrizer $R^{\prime}\left(\zeta^{\prime}, r\right)$ in the domain

$$
\Omega_{+}(0)=\Omega\left(\zeta_{0}=0\right) \cap\{\eta \mid \eta \geqslant 0\},
$$

we select a finite set of neighborhoods $\Omega\left(\zeta_{0}^{\prime}, 0\right)$ which cover the half sphere $\left|\xi^{\prime}\right|^{2}+\left|\eta^{\prime}\right|^{2}=1, \eta^{\prime} \geqslant 0$. Then using a subordinate partition of unity we "patch" together the local symbols $R^{\prime}\left(\zeta^{\prime}, r\right)$. The resulting symbol, still denoted by $R^{\prime}\left(\zeta^{\prime}, r\right)$, is a smooth function of $\zeta^{\prime}$ and $r$ and satisfies estimate (3.68) for any $\zeta \in \Omega_{+}(0)$.

3.3. Proof of Theorem 1.3. We consider only the more difficult case when $\zeta=(\xi, \eta)$ in problem (3.4) belongs to the neighborhood $\Omega_{+}(0)$ in (3.72). Introduce in (3.4) new variables

$$
u\left(x_{0}\right)=X(\zeta) v\left(x_{0}\right), \quad F\left(x_{0}\right)=T(\zeta) G\left(x_{0}\right),
$$

where $v\left(x_{0}\right)$ and $G\left(x_{0}\right)$ are partitioned in accordance with (3.25)

$$
v=\left(\begin{array}{c}
v_{f} \\
v_{\infty}
\end{array}\right), \quad v_{f}=\left(\begin{array}{c}
v_{0} \\
v_{1}
\end{array}\right), \quad G=\left(\begin{array}{c}
G_{f} \\
G_{\infty}
\end{array}\right), \quad G_{f}=\left(\begin{array}{c}
G_{0} \\
G_{1}
\end{array}\right) .
$$

In view of equivalence (3.26), problem (3.4) becomes

$$
\begin{array}{ll}
\text { (A) } & \left(E_{0}-M_{f}(\zeta)\right) v_{f}\left(x_{0}\right)=G_{f}\left(x_{0}\right), \\
\text { (B) }\left(-M_{\infty}(\zeta) E_{0}+I\right) v_{\infty}\left(x_{0}\right)=G_{\infty}\left(x_{0}\right), \\
\text { (C) } \tilde{S}(\zeta) X(\zeta) v(0)=g .
\end{array}
$$

We set

$$
\begin{aligned}
R_{0}(\zeta)=-c I, & R_{\infty}(\zeta)=I \\
R_{f}(\zeta)=\operatorname{diag}\left(R_{0}(\zeta), R^{\prime}\left(\zeta^{\prime}, r\right)\right), & R(\zeta)=\operatorname{diag}\left(R_{f}(\zeta), R_{\infty}(\zeta)\right)
\end{aligned}
$$


and multiply Eqs. (3.75)(A) and (B) in $l_{2}\left(x_{0}\right)$ by the grid functions

$$
R_{f}(\zeta)\left(-E_{0}-M_{f}(\zeta)\right) v_{f}\left(x_{0}\right) \text { and } R_{\infty}(\zeta)\left(M_{\infty}(\zeta) E_{0}+I\right) v_{\infty}\left(x_{0}\right)
$$

correspondingly. Then, comparing the real parts, we obtain

$$
\begin{gathered}
\left\langle\left(M_{f}^{*} R_{f} M_{f}-R_{f}\right) v_{f}, v_{f}\right\rangle+\left(R_{f} v_{f}(0), v_{f}(0)\right) h \\
=-\operatorname{Re}\left\langle R_{f}\left(E_{0}+M_{f}\right) v_{f}, G_{f}\right\rangle, \\
\left\langle\left(R_{\infty}-M_{\infty}^{*} R_{\infty} M_{\infty}\right) E_{0} v_{\infty}, E_{0} v_{\infty}\right\rangle+\left(R_{\infty} v_{\infty}(0), v_{\infty}(0)\right) h \\
=\operatorname{Re}\left\langle R_{\infty}\left(M_{\infty} E_{0}+I\right) v_{\infty}, G_{\infty}\right\rangle .
\end{gathered}
$$

Using estimates (3.21), (3.68) and Schwarz' inequality, we get

$$
\delta\left(\left\|v_{0}\right\|^{2}+\eta\left\|v_{1}\right\|^{2}\right)+\left(R_{f} v_{f}(0), v_{f}(0)\right) h \leqslant K\left(\left\|G_{0}\right\|^{2}+\left\|G_{1}\right\|^{2} \eta^{-1}\right)
$$

and

$$
\delta\left\|E_{0} v_{\infty}\right\|^{2}+\left(R_{\infty} v_{\infty}(0), v_{\infty}(0)\right) h \leqslant K\left\|G_{\infty}\right\|^{2},
$$

where $\|\cdot\|$ stands for the $l_{2}\left(x_{0}\right)$ norm $\|\cdot\|_{x_{0}}$. Since $R_{\infty}=I$, the last estimate is equivalent to

$$
\delta\left\|v_{\infty}\right\|^{2}+\left(R_{\infty} v_{\infty}(0), v_{\infty}(0)\right) h \leqslant K\left\|G_{\infty}\right\|^{2} .
$$

For $0<\eta \leqslant K_{0}$ we derive from (3.79) and (3.81)

$$
\delta \eta\|v\|^{2}+(R v(0), v(0)) h \leqslant K\|G\|^{2} \eta^{-1} .
$$

The following lemma enables us to estimate the second term in (3.82).

LeMma 3.3. Suppose that (UKC) holds for any $\zeta \in \Omega_{+}(0)$. Then, for a sufficiently small constant $c$ in (3.71) and (3.76) the symbol $R(\zeta)$ satisfies

$$
(R(\zeta) v(0), v(0)) \geqslant \delta|v(0)|^{2}-K|g|^{2},
$$

or, in other words,

$$
R(\zeta)+K[\tilde{S}(\zeta) X(\zeta)] *[\tilde{S}(\zeta) X(\zeta)] \geqslant \delta I
$$

for any $\zeta \in \Omega_{+}(0)$.

Proof. We can assume that the pair $\left(\zeta^{\prime}, r\right)$ corresponding to the point $\zeta \in \Omega_{+}(0)$ belongs to some neighborhood $\Omega\left(\zeta_{0}^{\prime}, 0\right)$ discussed in the previous subsection and that $R^{\prime}\left(\zeta^{\prime}, r\right)$ is a local symmetrizer in $\Omega\left(\zeta_{0}^{\prime}, 0\right)$. The "patching" together of the local symbols satisfying (3.84) will maintain this estimate. In view of estimate (3.71) and definition (3.76), for any vector $v$ partitioned as in (3.74) we have

$$
(R(\zeta) v, v) \geqslant\left|v_{\infty}\right|^{2}+\left|v_{\mathrm{II}}^{\prime}\right|^{2}-c\left(\left|v_{\mathrm{I}}^{\prime}\right|^{2}+\left|v_{0}\right|^{2}\right)
$$

where the vector $v^{\prime}$ is defined by

$$
v^{\prime}=\left(X^{\prime}\left(\zeta^{\prime}, r\right)\right)^{-1} v_{1}
$$

and is partitioned as in (3.69). Define $g=\tilde{S}(\zeta) X(\zeta) v$ and rewrite this equality as

$$
g=\tilde{S}_{\mathrm{I}}\left(\zeta^{\prime}, r\right)\left(\begin{array}{c}
v_{0} \\
v_{\mathrm{I}}^{\prime}
\end{array}\right)+\tilde{S}_{\mathrm{II}}\left(\zeta^{\prime}, r\right)\left(\begin{array}{c}
v_{\mathrm{II}}^{\prime} \\
v_{\infty}
\end{array}\right),
$$


where

$$
\begin{aligned}
& \tilde{S}_{\mathrm{I}}\left(\zeta^{\prime}, r\right)=\tilde{S}(\zeta)\left[X_{0}(\zeta), X_{\mathrm{I}}(\zeta) \cdot X_{\mathrm{I}}^{\prime}\left(\zeta^{\prime}, r\right)\right], \\
& \tilde{S}_{\mathrm{II}}\left(\zeta^{\prime}, r\right)=\tilde{S}(\zeta)\left[X_{\mathrm{I}}(\zeta) \cdot X_{\mathrm{II}}^{\prime}\left(\zeta^{\prime}, r\right), X_{\infty}(\zeta)\right] .
\end{aligned}
$$

In order to prove (3.83) it is enough to show that

$$
\operatorname{det} \tilde{S}_{\mathrm{I}}\left(\zeta^{\prime}, r\right) \neq 0 \quad \text { for any }\left(\zeta^{\prime}, r\right) \in \Omega\left(\zeta_{0}^{\prime}, 0\right) \text {. }
$$

Consider Eqs. (3.75)(A), (B) with $G=0$. Once again change variables

$$
v^{\prime}=U\left(\zeta^{\prime}, r\right) w,
$$

where $U=\operatorname{diag}\left(U_{1}, U_{2}, \ldots, U_{m}\right)$. Here the matrices $U_{j}\left(\zeta^{\prime}, r\right), j=1,2, \ldots, m$, are defined as in (3.38) if $\operatorname{Re} \kappa_{j}^{\prime}=0$ and $q_{j}>1$, and $U_{j}=I_{q_{j}}$ otherwise. Partition the vector $w$ as

$$
\left(\begin{array}{c}
w_{\mathrm{I}} \\
w_{\mathrm{II}}
\end{array}\right)
$$

in the same way as we did with $v^{\prime}$. Due to (3.38) Eqs. (3.75)(A) and (B) become

$$
\begin{aligned}
& \text { (A) }\left(E_{0}-M_{0}\right) v_{0}\left(x_{0}\right)=0, \\
& \text { (B) }\left(E_{0}-\left[\begin{array}{cc}
N_{11} & N_{12} \\
0 & N_{22}
\end{array}\right]\right)\left(\begin{array}{l}
w_{\mathrm{I}}\left(x_{0}\right) \\
w_{\mathrm{II}}\left(x_{0}\right)
\end{array}\right)=0, \\
& \text { (C) }\left(-M_{\infty} E_{0}+I\right) v_{\infty}\left(x_{0}\right)=0,
\end{aligned}
$$

where, for $\left(\zeta^{\prime}, r\right) \in \Omega_{+}\left(\zeta_{0}^{\prime}, 0\right)$ and $r>0$, the spectra of the matrices $N_{11}$ and $N_{22}$ lie correspondingly inside and outside the unit circle $|\kappa|=1$. Then the general solution of (3.89) in $l_{2}\left(x_{0}\right)$ is given by

$$
v_{\infty}\left(x_{0}\right)=w_{\mathrm{II}}\left(x_{0}\right)=0, \quad v_{0}(\nu h)=M_{0}^{\nu} v_{0}(0), \quad w_{\mathrm{I}}(\nu h)=N_{11}^{\nu} w_{\mathrm{I}}(0),
$$

and the corresponding homogeneous solution of Eq. (3.4)(A) is

$$
\begin{aligned}
\varphi\left(x_{0}, \zeta^{\prime}, r\right)= & \left(\varphi_{1}\left(x_{0}, \zeta^{\prime}, r\right), \ldots, \varphi_{l}\left(x_{0}, \zeta^{\prime}, r\right)\right) w_{\mathrm{I}}(0) \\
& +\left(\varphi_{l+1}\left(x_{0}, \zeta\right), \ldots, \varphi_{l_{0}}\left(x_{0}, \zeta\right)\right) v_{0}(0) \\
= & X_{1}(\zeta) X^{\prime}\left(\zeta^{\prime}, r\right) U\left(\zeta^{\prime}, r\right)\left(\begin{array}{c}
w_{\mathrm{I}}\left(x_{0}\right) \\
0
\end{array}\right)+X_{0}(\zeta) v_{0}\left(x_{0}\right) .
\end{aligned}
$$

The number of independent solutions in the first group is, indeed, $l$ as it is for the differential system (1.1). The matrix-function $U\left(\zeta^{\prime}, r\right)$ is continuous at the point $\left(\zeta_{0}^{\prime}, 0\right)$ and $U\left(\zeta_{0}^{\prime}, 0\right)=I$. Then, by $(3.24)$, we get

$$
\left(\varphi_{1}\left(0, \zeta_{0}^{\prime}, 0\right), \ldots, \varphi_{l}\left(0, \zeta_{0}^{\prime}, 0\right)\right)=\left(\begin{array}{c}
I \\
I \\
\vdots \\
I
\end{array}\right) X_{\mathrm{I}}^{\prime}\left(\zeta_{0}^{\prime}, 0\right)
$$

From (3.29) we deduce that the matrix $X^{\prime}\left(\zeta_{0}^{\prime}, 0\right)$ and the first $k$ components of the vectors $\varphi_{j}\left(0, \zeta^{\prime}, 0\right), j=1,2, \ldots, l$, are exactly the same as one obtains in the normal mode analysis for the differential problem (1.1)-(1.3). This remark justifies formula (1.38) and Lemma 1.2. Substitute the vectors $\varphi_{j}\left(0, \zeta_{0}^{\prime}, 0\right)$ from (3.91) and the vectors $\varphi_{l+1}(0,0), \ldots, \varphi_{l_{0}}(0,0)$ in the boundary condition $(3.4)(\mathrm{B})$. The resulting matrix coincides with the matrix $\left[N_{1}\left(x_{-}, t, \zeta_{0}^{\prime}, 0\right), N_{2}\left(x_{-}, t, \zeta=0\right)\right]$ in $(1.40)$ as well as with 
the matrix $\tilde{S}_{\mathrm{I}}\left(\zeta_{0}^{\prime}, 0\right)$ in (3.87). Thus (UKC) implies that $\operatorname{det} \tilde{S}_{\mathrm{I}}\left(\zeta_{0}^{\prime}, 0\right) \neq 0$ and therefore the inequality in (3.87) holds for any $\zeta^{\prime} \in \Omega\left(\zeta_{0}^{\prime}, 0\right)$, provided the neighborhood $\Omega\left(\zeta_{0}^{\prime}, 0\right)$ is sufficiently small. Q.E.D.

Return now to estimate (3.82). From (3.83) we get

$$
\eta\|v\|^{2}+h|v(0)|^{2} \leqslant K\left(\eta^{-1}\|G\|^{2}+h|g|^{2}\right)
$$

which is equivalent to estimate (3.5). Thus we have proved the sufficiency part of Theorem 1.3.

The necessity of (UKC) follows easily from the analysis in Lemma 3.3. Again, we consider the more difficult case when condition (1.40) is violated at the point $\zeta^{\prime}=\zeta_{0}^{\prime}, r=0$. Then det $\tilde{S}_{\mathrm{I}}\left(\zeta_{0}^{\prime}, 0\right)=0$, and there exists a nonzero vector $\left(v_{0}(0), w_{\mathrm{I}}(0)\right)$ such that

$$
\tilde{S}_{\mathrm{I}}\left(\zeta_{0}^{\prime}, 0\right)\left(\begin{array}{l}
v_{0}(0) \\
w_{\mathrm{I}}(0)
\end{array}\right)=0 \text {. }
$$

With the above vector we build as in (3.90) a homogeneous solution $\varphi\left(x_{0}, \zeta^{\prime}, r\right)$ of Eq. (3.4)(A). Then $\varphi\left(0, \zeta^{\prime}, r\right)$ satisfies the boundary condition

$$
\tilde{S}(\zeta) \varphi\left(0, \zeta^{\prime}, r\right)=g\left(\zeta^{\prime}, r\right),
$$

where $g\left(\zeta^{\prime}, r\right)$ is continuous at $\left(\zeta_{0}^{\prime}, 0\right)$ and $g\left(\zeta_{0}^{\prime}, 0\right)=0$. The last violates the estimate $\left|\varphi\left(0, \zeta^{\prime}, r\right)\right| \leqslant K\left|g\left(\zeta^{\prime}, r\right)\right|$ and, thus, estimate (3.5).

4. An Algebra of Pseudo-Difference Operators. In this section we study an algebra of pseudo-difference operators (later referred to as P.D.O.) depending on parameters $h$ and $\eta$ and acting in appropriate Sobolev spaces of grid vector-functions. Although similar algebras were built in [3] and [12], for completeness and rigor we present the proofs of all basic results of this section. These results will be used later in order to prove Theorems $1.1-1.3$ in the case of variable coefficients.

We introduce first a class $S_{1,0}^{m}$ of symbols $a(x, \xi, \eta)$ which are smooth functions of $x=\left(x_{1}, x_{2}, \ldots, x_{n}\right) \in R^{n}$ and $(\xi, \eta)=\left(\xi_{1}, \xi_{2}, \ldots, \xi_{n}, \eta\right) \in R^{n} \times\left[0, K_{0}\right] \backslash\{0\}$, are periodic in $\xi_{j}, j=1,2, \ldots, n$, with the period $2 \pi$, have a limit $a(\infty, \xi, \eta)$ as $x \rightarrow \infty$ and satisfy the estimates

$$
\left|(1+|x|)^{\gamma} D_{x}^{\beta} D_{\xi}^{\alpha} a^{\prime}(x, \xi, \eta)\right| \leqslant K_{\alpha, \beta, \gamma} \lambda^{m-|\alpha|}(\xi, \eta)
$$

and

$$
\left|D_{x}^{\beta} D_{\xi}^{\alpha} a(\infty, \xi, \eta)\right| \leqslant K_{\alpha, \beta} \lambda^{m-|\alpha|}(\xi, \eta)
$$

for any integer $\gamma$ and multi-indices $\alpha$ and $\beta$. Here

$$
\lambda(\xi, \eta)=\left(\sum_{j=1}^{n}\left|e^{i \xi_{j}}-1\right|^{2}+\left|e^{\eta}-1\right|^{2}\right)^{1 / 2}
$$

and

$$
a^{\prime}(x, \xi, \eta)=a(x, \xi, \eta)-a(\infty, \xi, \eta) .
$$

Obviously, $S_{1,0}=\Sigma_{m=-\infty}^{+\infty} S_{1,0}^{m}$ is a graduate algebra under the usual addition and multiplication of symbols. We also consider a subalgebra $\tilde{S}_{1,0}^{0} \subset S_{1,0}^{0}$ consisting of symbols $a(x, \xi, \eta)$ which satisfy the estimates

$$
\left|(1+|x|)^{\gamma} D_{x}^{\beta} D_{\xi}^{\alpha} a^{\prime}(x, \xi, \eta)\right| \leqslant K_{\alpha, \beta, \gamma},
$$




$$
\left|D_{x}^{\beta} D_{\xi}^{\alpha} a(\infty, \xi, \eta)\right| \leqslant K_{\alpha, \beta} .
$$

Following Frank [3], we define for $a(x, \xi, \eta) \in S_{1,0}^{m}$ the seminorms

$$
\begin{array}{r}
|a|_{m,|\alpha|, \beta}=\sum_{|\alpha|=\text { const }}\left[(2 \pi)^{-n} \int_{R^{n}} \sup _{\xi, \eta}\left|\left(1+|\chi|^{2}\right)^{\beta / 2} D_{\xi}^{\alpha} \hat{a}^{\prime}(\chi, \xi, \eta) \lambda^{|\alpha|-m}(\xi, \eta)\right| d \chi\right. \\
\left.+\sup _{\xi, \eta}\left|D_{\xi}^{\alpha} a(\infty, \xi, \eta) \lambda^{|\alpha|-m}(\xi, \eta)\right|\right] .
\end{array}
$$

Here $\hat{a}^{\prime}(\chi, \xi, \eta)=\int_{R^{n}} e^{-x \chi} a^{\prime}(x, \xi, \eta) d x$ is the Fourier transform of $a^{\prime}$. One can easily check that the above seminorms are bounded.

Now let $a(h, x, \xi, \eta)$ be a smooth function of $0<h<h_{0}, x \in R^{n}, \xi \in R^{n}$ and $\eta \in\left[0, K_{0} / h\right]$ periodic in $\xi_{j}, j=1,2, \ldots, n$, with a period $2 \pi / h$, which has a limit $a(h, \infty, \xi, \eta)$ as $x \rightarrow \infty$ and satisfies the estimates

$$
\left|\left(1+|x|^{\gamma}\right) D_{x}^{\beta} D_{\xi}^{\alpha} a^{\prime}(h, x, \xi, \eta)\right| \leqslant K_{\alpha, \beta, \gamma} h^{m_{2}} \Lambda^{m_{1}-|\alpha|}(h, \xi, \eta)
$$

and

$$
\left|D_{x}^{\beta} D_{\xi}^{\alpha} a(h, \infty, \xi, \eta)\right| \leqslant K_{\alpha, \beta} h^{m_{2}} \Lambda^{m_{1}-|\alpha|}(h, \xi, \eta)
$$

Here

$$
\Lambda(h, \xi, \eta)=\left(1+\sum_{j=1}^{n}\left|\frac{e^{i \xi_{j} h}-1}{h}\right|^{2}+\left|\frac{e^{\eta h}-1}{h}\right|^{2}\right)^{1 / 2}
$$

and $a^{\prime}(h, x, \xi, \eta)=a(h, x, \xi, \eta)-a(h, \infty, \xi, \eta)$. Frank in [3] uses for $\Lambda(h, \xi, 0)$ the notation $\left\langle\zeta_{\xi}\right\rangle$. Following [12] we denote the class of symbols $a(h, x, \xi, \eta)$ with given integers $m_{1}$ and $m_{2}$ by $S_{1,0}^{m_{1}, m_{2}}$. Our symbols differ from those in [12] by their dependence on $\eta$ and also periodicity in $\xi$ (instead of vanishing near $\xi_{j}= \pm \pi / h$ ) and in the last feature they resemble the symbols in [3]. As in [12] we define in addition subclasses $\tilde{S}_{1,0}^{0, m_{2}} \subset S_{1,0}^{0, m_{2}}$ of symbols $a(h, x, \xi, \eta)$ which satisfy estimates (4.7) and (4.8) with the right-hand side replaced by $K h^{m_{2}+|\alpha|}$. Obviously $\Sigma_{m_{1}, m_{2}} S_{1,0}^{m_{1}, m_{2}}$ and $\sum_{m_{2}} \tilde{S}_{1,0}^{0, m_{2}}$ are graduate algebras of symbols. For $a \in S_{1,0}^{m_{1}, m_{2}}$ we define seminorms

$$
\begin{aligned}
|a|_{m_{1}, m_{2},|\alpha|, \beta}= & \sum_{|\alpha|=\text { const }}(2 \pi)^{-n} \int_{R^{n}} \sup _{\xi, \eta, h} \mid\left(1+|\chi|^{2}\right)^{\beta / 2}\left(D_{\xi}^{\alpha} \hat{a}^{\prime}(h, \chi, \xi, \eta)\right) \\
& \quad \times h^{-m_{2}} \Lambda^{|\alpha|-m_{1}}(h, \xi, \eta) \mid d \chi \\
& +\sup _{\xi, \eta, h}\left|\left(D_{\xi}^{\alpha} a(h, \infty, \xi, \eta)\right) h^{-m_{2}} \Lambda^{|\alpha|-m_{1}}(h, \xi, \eta)\right|
\end{aligned}
$$

where $\hat{a}^{\prime}(h, \chi, \xi, \eta)$ is the Fourier transform of $a^{\prime}(h, x, \xi, \eta)$ in $x$ with the dual variable $\chi$ and the supremum is taken over $\xi \in R^{n}, \eta \in\left[0, K_{0} / h\right]$ and $h \in\left(0, h_{0}\right)$.

Remark 4.1. For $1 \leqslant \eta \leqslant K_{0} / h$ the function $\Lambda(h, \xi, \eta)$ is of the same order of magnitude as $\lambda(h \xi, h \eta) / h$. Therefore, given $a(x, \xi, \eta) \in S_{1,0}^{m}$ the corresponding symbol $a(x, h \xi, h \eta)$ restricted to $\eta \in\left[1, K_{0} / h\right]$ belongs to $S_{1,0}^{m, m}$ and

$$
|a(x, h \xi, h \eta)|_{m, m,|\alpha|, \beta} \leqslant K|a(x, \xi, \eta)|_{m,|\alpha|, \beta}
$$


However, if $a(x, \xi, \eta) \in \tilde{S}_{1,0}^{0}$, the symbol $a(x, h \xi, h \eta)$ belongs to $\tilde{S}_{1,0}^{0,0}$ for $\eta \in$ $\left[0, K_{0} / h\right]$.

Let $u(x)$ be a grid function defined on a uniform grid $R_{h}^{n}$ such that $u(x) \in e^{\eta x_{n}} l_{2}(x)$ (we use notations of Section 1 with $t=x_{n}$ ). Denote by $T_{h}^{n}$ the $n$-dimensional cube $\left\{\xi \in R^{n}|| \xi_{j} \mid \leqslant \pi / h, j=1,2, \ldots, n\right\}$. We define the norm

$$
\|u\|_{k, \eta, h}^{2}=(2 \pi)^{-n} \int_{\xi \in T_{h}^{n}}\left|\hat{u}^{h}(\xi, \eta)\right|^{2} \Lambda^{2 k}(h, \xi, \eta) d \xi
$$

where

$$
\hat{u}^{h}(\xi, \eta)=\mathscr{F}_{x \rightarrow \xi, h}\left(u(x) e^{-\eta x_{n}}\right)=\sum_{x \in R_{h}^{n}} h^{n} u(x) e^{-\eta x_{n}} e^{-i \xi x}
$$

is the discrete Fourier transform of $u(x) e^{-\eta x_{n}}$, and denote by $H_{k, \eta, h}$ the corresponding space of grid functions. Obviously, for fixed $h>0$ the norms $\|u\|_{k, \eta, h}$ are equivalent to $\|u\|_{0, \eta, h}$. Note also that the norm $\|u\|_{0, \eta, h}$ coincides with the one defined in (1.11) and thus $H_{0, \eta, h}=e^{\eta x_{n}} l_{2}(x)$. To a symbol $a(h, x, \xi, \eta) \in S_{1,0}^{m_{1}, m_{2}}$ we relate a two-parameter family of P.D.O. $A_{\eta}^{h}$ acting on grid functions $u \in H_{0, \eta, h}$ and defined by

$$
\left(A_{\eta}^{h} u\right)(x)=(2 \pi)^{-n} \int_{\xi \in T_{h}^{n}} e^{i x \xi+x_{n} \eta} a(h, x, \xi, \eta) \hat{u}^{h}(\xi, \eta) d \xi .
$$

We shall also denote such an operator by $a(h, x, D, \eta)$ or, instead, write

$$
\sigma\left(A_{\eta}^{h}\right)=a(h, x, \xi, \eta) .
$$

To shorten the notation we shall often replace the pairs $(\xi, \eta),\left(\xi^{\prime}, \eta\right)$ and $\left(\xi^{\prime \prime}, \eta\right)$ by the letters $\zeta, \zeta^{\prime}$ and $\zeta^{\prime \prime}$. The definition in (4.13) implies

$$
\begin{aligned}
& \left(\widehat{A}_{\eta}^{h} u^{h}\right)\left(\zeta^{\prime}\right)=(2 \pi)^{-n} \int_{T_{h}^{n}} \widehat{a}^{\prime h}\left(h, \xi^{\prime}-\xi, \zeta\right) \hat{u}^{h}(\zeta) d \xi \\
& +a\left(h, \infty, \zeta^{\prime}\right) \hat{u}^{h}\left(\zeta^{\prime}\right),
\end{aligned}
$$

where $\widehat{a}^{\prime h}\left(h, \xi^{\prime}-\xi, \zeta\right)=\mathscr{F}_{x \rightarrow \xi^{\prime}-\xi, h}\left(a^{\prime}(h, x, \zeta)\right)$ is the discrete Fourier transform of the function $a^{\prime}(h, x, \zeta)$. By the Poisson summation formula (see [2]),

$$
\widehat{a}^{\prime h}(h, \chi, \zeta)=\sum_{\alpha \in \mathbf{Z}^{n}} \widehat{a^{\prime}}\left(h, \chi+2 \pi \alpha h^{-1}, \zeta\right),
$$

where $\widehat{a^{\prime}}(h, \chi, \zeta)$ is the continuous Fourier transform of $a^{\prime}(h, x, \zeta)$ in $x \in R^{n}$ with the dual variable $\chi$.

THEOREM 4.1. For $a(h, x, \zeta) \in S_{1,0}^{m, 0}$ the norm of the operators $A_{\eta}^{h}: H_{k+m, \eta, h} \rightarrow H_{k, \eta, h}$ is bounded by $2^{|k| / 2}|a|_{m, 0,0,|k|}$.

Proof. For simplicity we consider only the case $a(h, \infty, \zeta)=0$. In view of (4.14) we have

$$
\begin{aligned}
\left|\Lambda^{k}\left(h, \zeta^{\prime}\right)\left(\widehat{A}_{\eta}^{h} u^{h}\right)\left(\zeta^{\prime}\right)\right| & \\
\leqslant(2 \pi)^{-n} \int_{T_{h}^{n}} \mid\left[\Lambda ^ { k } ( h , \zeta ^ { \prime } ) \Lambda ^ { - k } ( h , \zeta ) \hat { a } ^ { h } \left(h, \xi^{\prime}\right.\right. & \left.-\xi, \zeta) \Lambda^{-m}(h, \zeta)\right] \\
& \times \Lambda^{k+m}(h, \zeta) \hat{u}^{h}(\zeta) \mid d \xi .
\end{aligned}
$$


Due to the estimate

$$
\Lambda(h, \zeta) \Lambda^{-1}\left(h, \zeta^{\prime}\right) \leqslant 2^{1 / 2} \Lambda\left(h, \xi-\xi^{\prime}, 0\right),
$$

which follows from Peetre's inequality, the expression in the square brackets in the above integral is bounded by a function

$$
b\left(h, \xi^{\prime}-\xi, \zeta\right)=2^{|k| / 2} \Lambda^{|k|}\left(h, \xi^{\prime}-\xi, 0\right)\left|\hat{a}^{h}\left(h, \xi^{\prime}-\xi, \zeta\right)\right| \Lambda^{-m}(h, \zeta) .
$$

Then, by Young's inequality, formula (4.15) and periodicity of $\Lambda^{k}(h, \chi, 0)$ in $\chi$ we get

$$
\begin{aligned}
\left\|A_{\eta}^{h}\right\| & \leqslant(2 \pi)^{-n} \int_{T_{h}^{n}} \sup _{\zeta, h} b(h, \chi, \zeta) d \chi \\
& \leqslant(2 \pi)^{-n} 2^{|k| / 2} \int_{R_{h}^{n}} \sup _{\zeta, h} \Lambda^{|k|}(h, \chi, 0)|\hat{a}(h, \chi, \zeta)| \Lambda^{-m}(h, \zeta) d \chi .
\end{aligned}
$$

Since for $\chi \in R^{n}$,

$$
\Lambda(h, \chi, 0) \leqslant\left(1+|\chi|^{2}\right)^{1 / 2},
$$

the last integral is bounded by $2^{|k| / 2}|a|_{m, 0,0,|k|}$. Q.E.D.

TheOREM 4.2. Let $A_{\eta}^{h}, B_{\eta}^{h}$ and $C_{\eta}^{h}$ be families of P.D.O. with the symbols $a(h, x, \zeta)$ $\in S_{1,0}^{m_{1}, 0}, b(h, x, \zeta) \in S_{1,0}^{m_{2}, 0}$ and $c(h, x, \zeta)=a(h, x, \zeta) \cdot b(h, x, \zeta) \in S_{1,0}^{m_{1}+m_{2}, 0}$. Then the norms of the operators

$$
A_{\eta}^{h} \circ B_{\eta}^{h}-C_{\eta}^{h}: H_{k+m_{1}+m_{2}, \eta, h} \rightarrow H_{k+1, \eta, h}
$$

are bounded by

$$
\left\|A_{\eta}^{h} \circ B_{\eta}^{h}-C_{\eta}^{h}\right\| \leqslant K|a|_{m_{1}, 0,1,|k+1|} \cdot|b|_{m_{2}, 0,0,|k+1|+\left|m_{1}-1\right|+1} .
$$

Proof. Again we consider only the case $a(h, \infty, \zeta)=b(h, \infty, \zeta)=0$. Let $u \in$ $H_{k+m_{1}+m_{2}, \eta, h}$ and $v=\left(A_{\eta}^{h} \circ B_{\eta}^{h}-C_{\eta}^{h}\right) u$. Then

(4.19) $\Lambda^{k+1}\left(h, \zeta^{\prime}\right) \hat{v}^{h}\left(\zeta^{\prime}\right)$

$$
\begin{gathered}
=(2 \pi)^{-2 n} \int_{T_{h}^{n}}\left\{\int_{T_{h}^{n}}\left[\Lambda^{k+1}\left(h, \zeta^{\prime}\right) \Lambda^{-k-1}(h, \zeta)\right]\right. \\
\cdot\left[\Lambda ^ { - m _ { 1 } + 1 } ( h , \zeta ) \left(\hat{a}^{h}\left(h, \xi^{\prime}-\xi^{\prime \prime}, \zeta^{\prime \prime}\right)\right.\right. \\
\left.\left.-\hat{a}^{h}\left(h, \xi^{\prime}-\xi^{\prime \prime}, \zeta\right)\right) \Lambda^{-1}\left(h, \zeta^{\prime \prime}-\zeta\right)\right] \\
\left.\cdot\left[\Lambda\left(h, \zeta^{\prime \prime}-\zeta\right) \hat{b}^{h}\left(h, \xi^{\prime \prime}-\xi, \zeta\right) \Lambda^{-m_{2}}(h, \zeta)\right] d \xi^{\prime \prime}\right\} \\
\cdot\left[\Lambda^{m_{1}+m_{2}+k}(h, \zeta) \hat{u}^{h}(\zeta)\right] d \xi .
\end{gathered}
$$

The expression in the first square brackets in the above integral is bounded by (4.20) $2^{|k+1| / 2} \Lambda^{|k+1|}\left(h, \zeta^{\prime}-\zeta\right) \leqslant 2^{|k+1|} \Lambda^{|k+1|}\left(h, \zeta^{\prime}-\zeta^{\prime \prime}\right) \cdot \Lambda^{|k+1|}\left(h, \zeta^{\prime \prime}-\zeta\right)$. 
For the second brackets we have the following estimates:

$$
\left|\hat{a}^{h}\left(h, \chi, \zeta^{\prime \prime}\right)-\hat{a}^{h}(h, \chi, \zeta)\right| \leqslant \sum_{i=1}^{n} \sup _{0 \leqslant t \leqslant 1}\left|\hat{a}_{i}^{h}\left(h, \chi, \theta_{t}\right)\right| \cdot \widehat{\left|\xi^{\prime \prime}-\xi\right|},
$$

where $\theta_{t}=\zeta+\left(\widetilde{\xi^{\prime \prime}-\xi}, 0\right) t, \widetilde{\xi^{\prime \prime}-\xi}$ belongs to $T_{h}^{n}$ and equals $\xi^{\prime \prime}-\xi \bmod 2 \pi / h$, and $a_{i}(h, x, \zeta)=D_{\xi_{i}} a(h, x, \zeta)$;

$$
\begin{aligned}
& \Lambda^{-m_{1}+1}(h, \zeta) \leqslant 2^{\left|m_{1}-1\right| / 2} \Lambda^{-m_{1}+1}\left(h, \theta_{t}\right) \Lambda^{\left|m_{1}-1\right|}\left(h, \zeta-\theta_{t}\right) \\
& \leqslant 2^{\left|m_{1}-1\right| / 2} \Lambda^{-m_{1}+1}\left(h, \theta_{t}\right) \Lambda^{\left|m_{1}-1\right|}\left(h, \zeta^{\prime \prime}-\zeta\right), \\
&\left|\widetilde{\xi^{\prime \prime}-\xi}\right| \Lambda^{-1}\left(h, \zeta^{\prime \prime}-\zeta\right) \leqslant \pi / 2, \text { since }\left|\varphi /\left(e^{i \varphi}-1\right)\right| \leqslant \pi / 2 \text { for }|\varphi| \leqslant \pi
\end{aligned}
$$

Define the functions

$$
f(\chi)=(2 \pi)^{-n} \sum_{i=1}^{n} \sup _{\zeta, h}\left|\Lambda^{|k+1|}(h, \chi, 0) \hat{a}_{i}^{h}(h, \chi, \zeta) \Lambda^{-m_{1}+1}(h, \zeta)\right|
$$

and

$$
g(\chi)=(2 \pi)^{-n} \sup _{\zeta, h}\left|\Lambda^{|k+1|+\left|m_{1}-1\right|+1}(h, \chi, 0) \hat{b}^{h}(h, \chi, \zeta) \Lambda^{-m_{2}}(h, \zeta)\right| .
$$

Due to estimates (4.20)-(4.23),

$$
\begin{aligned}
& \left|\Lambda^{k+1}\left(h, \zeta^{\prime}\right) \hat{v}^{h}(\zeta)\right| \\
& \quad \leqslant K \int_{T_{h}^{n}}\left[\int_{T_{h}^{n}} f\left(\xi^{\prime}-\xi^{\prime \prime}\right) g\left(\xi^{\prime \prime}-\xi\right) d \xi^{\prime \prime}\right] \cdot\left[\Lambda^{m_{1}+m_{2}+k}(h, \zeta)\left|\hat{u}^{h}(\zeta)\right|\right] d \xi
\end{aligned}
$$

where $K=2^{|k+1|} 2^{\left|m_{1}-1\right| / 2} \pi / 2$. By Poisson's summation formula for $a_{i}$ and $b$ and (4.17) we get

$$
\int_{T_{h}^{n}} f(\chi) d \chi \leqslant|a|_{m_{1}, 0,1,|k+1|} \text { and } \int_{T_{h}^{n}} g(\chi) d \chi \leqslant|b|_{m_{2}, 0,0,|k+1|+\left|m_{1}-1\right|+1}
$$

Estimate (4.18) follows at once from (4.24) and the Young inequality.

Let $A_{\eta}^{h}$ be a family of P.D.O. with the symbol $a(h, x, \zeta) \in S_{1,0}^{m, 0}$ and $A_{\eta}^{(*) h}$ a family with the adjoint symbol $a^{*}(h, x, \zeta)$. Denote by $\left(A_{\eta}^{h}\right)^{*}$ the operator which is adjoint to $A_{\eta}^{h}$ in $H_{0, \eta, h}$, i.e. $\left(A_{\eta}^{h} u, v\right)_{0, \eta, h}=\left(u,\left(A_{\eta}^{h}\right)^{*} v\right)_{0, \eta, h}$ for any $u, v \in H_{0, \eta, h}$. Note that for fixed $h$ and $\eta$ the operators $A_{\eta}^{h}, A_{\eta}^{(*) h},\left(A_{\eta}^{h}\right)^{*}: H_{k_{1}, \eta, h} \rightarrow H_{k_{2}, \eta, h}$ are bounded for any $k_{1}$ and $k_{2}$.

THEOREM 4.3. The norm of the operators $\left(A_{\eta}^{h}\right)^{*}-A_{\eta}^{(*) h}: H_{k+m, \eta, h} \rightarrow H_{k+1, \eta, h}$ is bounded by $K|a|_{m, 0,1,|k+1|+|m-1|+1}$, where $K$ depends only on $m$ and $k$.

Proof. Let $u \in H_{k+m, \eta, h}$ and $v=\left[\left(A_{\eta}^{h}\right)^{*}-A_{\eta}^{(*) h}\right] u$. Then

$$
\begin{aligned}
\Lambda^{k+1}\left(h, \zeta^{\prime}\right) \hat{v}^{h}\left(\zeta^{\prime}\right)= & (2 \pi)^{-n} \int_{T_{h}^{n}}\left[\Lambda^{k+1}\left(h, \zeta^{\prime}\right) \Lambda^{-k-1}(h, \zeta)\right] \\
& \cdot\left[\Lambda^{-m-1}(h, \zeta)\left(\widehat{a}^{h}\left(h, \xi^{\prime}-\xi, \zeta^{\prime}\right)-\widehat{a}^{h}\left(h, \xi^{\prime}-\xi, \zeta\right)\right)\right] \\
& \cdot\left[\Lambda^{k+m}(h, \zeta) \hat{u}(\zeta)\right] d \xi .
\end{aligned}
$$


The terms in the first and the second brackets are estimated as in (4.20)-(4.23). Introduce the function

$$
f(\chi)=(2 \pi)^{-n} \sum_{i=1}^{n} \sup _{\zeta, h}\left|\Lambda^{|k+1|+|m-1|+1}(h, \chi, 0){\widehat{a_{i}^{*}}}^{h}(h, \chi, \zeta) \Lambda^{-m+1}(h, \zeta)\right| .
$$

Then

$$
\left|\Lambda^{k+1}\left(h, \zeta^{\prime}\right) \hat{v}^{h}\left(\zeta^{\prime}\right)\right| \leqslant K \int_{T_{h}^{n}} f\left(\xi^{\prime}-\xi\right) \Lambda^{k+m}(h, \zeta)\left|\hat{u}^{h}(\zeta)\right| d \xi,
$$

where $K=2^{(|k+1|+|m-1|) / 2} \pi / 2$. The theorem follows from the Young inequality and the estimate

$$
\int_{T_{h}^{n}} f(\chi) d \chi \leqslant|a|_{m, 0,1,|k+1|+|m-1|+1} .
$$

We need a modification of Theorems 4.2 and 4.3 for the case $a \in \tilde{S}_{1,0}^{0,0}$.

THEOREM 4.2a. Let $A_{\eta}^{h}, B_{\eta}^{h}, C_{\eta}^{h}$ be as in Theorem 4.2 and $a(h, x, \zeta) \in \tilde{S}_{1,0}^{0,0}$. Then the norm of the operators $A_{\eta}^{h} \circ B_{\eta}^{h}-C_{\eta}^{h}: H_{k+m_{2}, \eta, h} \rightarrow H_{k, \eta, h}$ is bounded by

$$
K h \sum_{i=1}^{n}\left|h^{-1} D_{\xi_{i}} a(h, x, \xi)\right|_{0,0,0,|k|} \cdot|b|_{m_{2}, 0,0,|k|+1} \text {. }
$$

In the proof of Theorem 4.2 one should only replace $k+1$ by $k,-m_{1}+1$ by 0 and then observe that the norm $\left|h^{-1} D_{\xi_{i}} a(h, x, \xi)\right|_{0,0,0,|k|}$ is bounded.

TheOREM 4.3a. Let $a(h, x, \zeta)$ in Theorem 4.3 belong to $\tilde{S}_{1,0}^{0,0}$. Then the norm of the operators $\left(A_{\eta}^{h}\right)^{*}-A_{\eta}^{(*) h}: H_{k, \eta, h} \rightarrow H_{k, \eta, h}$ is bounded by

$$
K h \sum_{i=1}^{n}\left|h^{-1} D_{\xi_{i}} a(h, x, \zeta)\right|_{0,0,0,|k|+1}
$$

The next result is known as a weak form of Gårding's inequality.

THEOREM 4.4. Let the symbol $p(h, x, \zeta)$ belong to $S_{1,0}^{0,0}$ and satisfy

$$
\operatorname{Re} p(h, x, \zeta) \geqslant \delta>0,
$$

and let $P_{\eta}^{h}$ be the corresponding family of P.D.O. Then

$$
\operatorname{Re}\left(P_{\eta}^{h} u, u\right)_{0, \eta, h} \geqslant(\delta / 2)\|u\|_{0, \eta, h}^{2}-K\|u\|_{-1 / 2, \eta, h}^{2},
$$

where the constant $K$ does not depend on $\eta, h$ and $u$.

The proof is standard. There exists a symbol $b(h, x, \zeta) \in S_{1,0}^{0,0}$ such that $b^{*}(h, x, \zeta) b(h, x, \zeta)=\operatorname{Re} p(h, x, \zeta)-\delta / 2$. Let $B_{\eta}^{h}$ and $R_{\eta}^{h}$ be families of P.D.O. with the symbols $b(h, x, \zeta)$ and $\operatorname{Re} p(h, x, \zeta)$, correspondingly, and $Q_{\eta}^{h}=\left[P_{\eta}^{h}+\left(P_{\eta}^{h}\right)^{*}\right] / 2$. Note that the norm of the operators $\Lambda^{1 / 2}(h, D, \eta)$ $\circ\left(Q_{\eta}^{h}-R_{\eta}^{h}\right) \circ \Lambda^{1 / 2}(h, D, \eta)$ acting from $H_{-1 / 2, \eta, h}$ to $H_{-1 / 2, \eta, h}$ is uniformly bounded. Therefore

$$
\left|\left(Q_{\eta}^{h} u, u\right)_{0, \eta, h}-\left(R_{\eta}^{h} u, u\right)_{0, \eta, h}\right| \leqslant K\|u\|_{-1 / 2, \eta, h}^{2} .
$$

Similarly, we get

$$
\left|\left(B_{\eta}^{h} u, B_{\eta}^{h} u\right)_{0, \eta, h}-\left(\left[R_{\eta}^{h}-\delta / 2\right] u, u\right)_{0, \eta, h}\right| \leqslant K\|u\|_{-1 / 2, \eta, h}^{2} .
$$


Then

$$
\operatorname{Re}\left(P_{\eta}^{h} u, u\right)_{0, \eta, h} \geqslant(\delta / 2)\|u\|_{0, \eta, h}^{2}+\left\|B_{\eta}^{h} u\right\|_{0, \eta, h}^{2}-K\|u\|_{-1 / 2, \eta, h}^{2} \text { Q.E.D. }
$$

THEOREM 4.4a. If $p(h, x, \zeta)$ is as in Theorem 4.4 and belongs to $\tilde{S}_{1,0}^{0,0}$, then

$$
\operatorname{Re}\left(P_{\eta}^{h} u, u\right)_{0, \eta, h} \geqslant(\delta / 2-K h)\|u\|_{0, \eta, h}^{2} .
$$

One should only observe that the symbol $b(h, x, \zeta)$ in Theorem 4.4 belongs to $\tilde{S}_{1,0}^{0,0}$ and use Theorems 4.2a and 4.3a to modify estimates (4.25) and (4.26).

We also need a local form of Gårding's inequality.

THEOREM 4.5. Let $p(h, x, \zeta)$ be a symbol in $S_{1,0}^{0,0}$ and $\varphi(x, \zeta) \in \tilde{S}_{1,0}^{0}$ be a scalar real function such that $\operatorname{Re} p(h, x, \zeta) \geqslant \delta I>0$ for $(x, h \zeta)$ belonging to an open set $\Omega$ supp $\varphi(x, \zeta)$. Denote by $P_{\eta}^{h}$ and $\Phi_{\eta}^{h}$ the families of P.D.O. with the symbols $p(h, x, \zeta)$ and $\varphi(x, h \zeta)$ correspondingly. Then

$$
\operatorname{Re}\left(P_{\eta}^{h} \circ \Phi_{\eta}^{h} u, \Phi_{\eta}^{h} u\right)_{0, \eta, h} \geqslant(\delta / 2)\left\|\Phi_{\eta}^{h} u\right\|_{0, \eta, h}^{2}-K\|u\|_{-1 / 2, \eta, h}^{2}
$$

Proof. As in the proof of Theorem 4.4 we define a symbol $b(h, x, \zeta) \in S_{1,0}^{0,0}$ such that $b^{*}(h, x, \zeta) b(h, x, \zeta)=\operatorname{Re} p(h, x, \zeta)-\delta / 2$ for $(x, h \zeta) \in \operatorname{supp} \varphi(x, \zeta)$. Let $P_{1, \eta}^{h}=\left(B_{\eta}^{h}\right)^{*} B_{\eta}^{h}+(\delta / 2) I$. Then $P_{1, \eta}^{h}$ is selfadjoint and $P_{1, \eta}^{h} \geqslant(\delta / 2) I$. Estimate (4.27) now follows from the fact that the operators

$$
\left(\operatorname{Re} P_{\eta}^{h}-P_{1, \eta}^{h}\right) \Phi_{\eta}^{h}: H_{k, \eta, h} \rightarrow H_{k+1, \eta, h}
$$

are uniformly bounded.

THEOREM 4.5a. Let $p(h, x, \zeta), \varphi(x, \zeta)$ be as in Theorem 4.5 and $p(h, x, \zeta) \in \tilde{S}_{1,0}^{0,0}$. Then

$$
\operatorname{Re}\left(P_{\eta}^{h} \circ \Phi_{\eta}^{h} u, \Phi_{\eta}^{h} u\right)_{0, \eta, h} \geqslant(\delta / 2)\left\|\Phi_{\eta}^{h} u\right\|_{0, \eta, h}^{2}-K h\|u\|_{0, \eta, h}^{2} .
$$

We should only indicate that $b(h, x, \zeta)$ belongs to $\tilde{S}_{1,0}^{0,0}$, and therefore the norms of the operators $\left(\operatorname{Re} P_{\eta}^{h}-P_{1, \eta}^{h}\right) \Phi_{\eta}^{h}: H_{k, \eta, h} \rightarrow H_{k, \eta, h}$ are bounded by $K h$.

Finally we state the sharp form of Gårding's inequality.

THEOREM 4.6. Let the symbol $p(h, x, \zeta) \in S_{1,0}^{1,0}$ be Hermitian and nonnegative. Then, for $0 \leqslant \eta \leqslant K_{0} / h, 0<h<h_{0}$ with $h_{0}$ sufficiently small, there exists a constant $K$ independent on $\eta$ and $h$ such that

$$
\operatorname{Re}\left(P_{\eta}^{h} u, u\right)_{0, \eta, h} \geqslant-K\|u\|_{0, \eta, h}^{2} .
$$

Proof. We shall modify and simplify the proof of this inequality given in $[3$, Theorem 8.2] for the case $\eta=0$. Let $\varphi(\theta) \in C_{0}^{\infty}\left(R^{n}\right)$ be a nonnegative even function with the support in the ball $|\theta| \leqslant 1$ such that

$$
\int_{R^{n}} \varphi^{2}(\theta) d \theta=1
$$

Following Frank in [3] we define

$$
\varphi_{h}\left(\zeta, \zeta^{\prime}\right)=\Lambda^{-n / 4}(h, \zeta) \varphi\left(\Lambda^{-1 / 2}(h, \zeta)\left(\xi^{\prime}-\xi\right)\right),
$$


and

$$
\Phi\left(h, \zeta, \zeta^{\prime}\right)=\sum_{\alpha} \varphi_{h}\left(\zeta, \zeta^{\prime}+2 \pi h^{-1} \alpha, \eta\right), \quad \alpha \in \mathbf{Z}^{n}
$$

Note that for $h$ sufficiently small only one term in the above sum is nonzero. Obviously, the function $\Phi\left(h, \zeta, \zeta^{\prime}\right)$ is periodic in $\xi$ and $\xi^{\prime}$ with the period $2 \pi / h$. Introduce the regularized symbol

$$
\begin{aligned}
a(h, x, \zeta) & =\int_{T_{h}^{n}} p\left(h, x, \zeta^{\prime}\right) \Phi^{2}\left(h, \zeta, \zeta^{\prime}\right) d \xi^{\prime} \\
& =\int_{R^{n}} p\left(h, x, \zeta^{\prime}\right) \varphi_{h}^{2}\left(\zeta, \zeta^{\prime}\right) d \zeta^{\prime} \\
& =\int_{R^{n}} p\left(h, x, \xi+\Lambda^{1 / 2}(h, \zeta) \theta, \eta\right) \varphi^{2}(\theta) d \theta
\end{aligned}
$$

and the double symbol

$$
b\left(h, \zeta, x, \zeta^{\prime}\right)=\int_{T_{h}^{n}} \Phi\left(h, \zeta, \zeta^{\prime \prime}\right) p\left(h, x, \zeta^{\prime \prime}\right) \Phi\left(h, \zeta^{\prime}, \zeta^{\prime \prime}\right) d \xi^{\prime \prime}
$$

Let $A_{\eta}^{h}$ be a family of P.D.O. with the symbol $a(h, x, \zeta)$ and the family $B_{\eta}^{h}$ be defined by

$$
\begin{aligned}
\left(\widehat{B}_{\eta}^{h}{ }^{h}\right)(\zeta)= & (2 \pi)^{-n} \int_{T_{h}^{n}}{\widehat{b^{\prime}}}^{h}\left(h, \zeta, \xi-\xi^{\prime}, \zeta^{\prime}\right) \hat{u}^{h}\left(\zeta^{\prime}\right) d \xi^{\prime} \\
& +b(h, \zeta, \infty, \zeta) \hat{u}^{h}(\zeta),
\end{aligned}
$$

where $b\left(h, \zeta, \infty, \zeta^{\prime}\right)$ is obtained by substitution $x=\infty$ in (4.34) and

$$
{\widehat{b^{\prime}}}^{h}\left(h, \zeta, \chi, \zeta^{\prime}\right)=\mathscr{F}_{x \rightarrow \chi, h}\left(b\left(h, \zeta, x, \zeta^{\prime}\right)-b\left(h, \zeta, \infty, \zeta^{\prime}\right)\right)
$$

We restrict the operators $A_{\eta}^{h}, B_{\eta}^{h}$ and $P_{\eta}^{h}$ to grid functions with finite support. Then for arbitrary $u \in H_{0, \eta, h}$ estimate (4.29) will follow by the usual approximation arguments. As in [3] the proof of the theorem consists of the following three lemmas.

LEMMA 4.6.1. The operators $B_{\eta}^{h}$ are selfadjoint in $H_{0, \eta, h}$ and satisfy

$$
\left(B_{\eta}^{h} u, u\right)_{0, \eta, h} \geqslant 0 \text {. }
$$

LEMMA 4.6.2. There exists a constant $K$ independent on $\eta$ and $h$ such that

$$
\left|\operatorname{Re}\left(\left[A_{\eta}^{h}-P_{\eta}^{h}\right] u, u\right)_{0, \eta, h}\right| \leqslant K\|u\|_{0, \eta, h}^{2}
$$

LEMMA 4.6.3. There exists a constant $K$ independent on $\eta$ and $h$ such that

$$
\left|\left(\left[B_{\eta}^{h}-\operatorname{Re} A_{\eta}^{h}\right] u, u\right)_{0, \eta, h}\right| \leqslant K\|u\|_{0, \eta, h}^{2} .
$$

Proof of Lemma 4.6.1. Since $b\left(h, \zeta, x, \zeta^{\prime}\right)=b^{*}\left(h, \zeta^{\prime}, x, \zeta\right)$, the operator $B_{\eta}^{h}$ is, obviously, selfadjoint. Consider the function

$$
F(x)=\int_{T_{h}^{n}} \int_{T_{h}^{n}}\left(b\left(h, \zeta, x, \zeta^{\prime}\right) e^{i x \xi^{\prime}} \hat{u}^{h}\left(\zeta^{\prime}\right), e^{i x \xi} \hat{u}^{h}(\zeta)\right) d \xi d \xi^{\prime}
$$


According to (4.34) we have

$$
F(x)=\int_{T_{h}^{n}}\left(p\left(h, x, \zeta^{\prime \prime}\right) v\left(\zeta^{\prime \prime}\right), v\left(\zeta^{\prime \prime}\right)\right) d \xi^{\prime \prime} \geqslant 0,
$$

where $v\left(\zeta^{\prime \prime}\right)=\int_{T_{h}^{n}} \Phi\left(h, \zeta, \zeta^{\prime \prime}\right) e^{i x \xi} \hat{u}^{h}(\zeta) d \xi$. It is easy to show that the partial sums

$$
\sum_{x} F(x) h^{n}, \quad x \in R_{h}^{n},\left|x_{j}\right| \leqslant N h, j=1,2, \ldots, n,
$$

converge to $(2 \pi)^{n}\left(\left(\widehat{B}_{\eta}^{h}{ }^{h}\right)(\zeta), \hat{u}^{h}(\zeta)\right)$ as $N \rightarrow \infty$. Thus,

$$
\left(B_{\eta}^{h} u, u\right)_{0, \eta, h}=(2 \pi)^{-n}\left(\left(\widehat{B}_{\eta}^{h} u^{h}\right)(\zeta), \hat{u}^{h}(\zeta)\right) \geqslant 0 .
$$

Proof of Lemma 4.6.2. The norm of the operators

$$
A_{\eta}^{h}-P_{\eta}^{h}: H_{0, \eta, h} \rightarrow H_{0, \eta, h}
$$

is bounded by

$$
K=|a(h, x, \zeta)-p(h, x, \zeta)|_{0,0,0,0}
$$

Using definition (4.33) and the fact that $\varphi(\theta)$ is an even function, we get as in [3]

(4.39) $a(h, x, \zeta)-p(h, x, \zeta)$

$$
\begin{aligned}
= & \int_{|\theta|<1} \int_{0}^{1} \Lambda(h, \zeta)(1-t) \\
& \times \sum_{|\alpha|=2} D_{\xi}^{\alpha} p\left(h, x, \xi+t \Lambda^{1 / 2}(h, \zeta) \theta, \eta\right) \theta^{\alpha} \varphi^{2}(\theta) d t d \theta .
\end{aligned}
$$

We shall show that for $\left|\xi^{\prime}-\xi\right|<\Lambda^{1 / 2}(h, \zeta)$ the ratio $\Lambda(h, \zeta) / \Lambda\left(h, \zeta^{\prime}\right)$ is bounded by a generic constant $c_{0}$. Indeed, the gradient $D_{\xi} \Lambda(h, \zeta)$ is bounded by 1 . Therefore

$$
\left|\Lambda(h, \zeta)-\Lambda\left(h, \zeta^{\prime}\right)\right| \leqslant\left|\xi-\xi^{\prime}\right| \leqslant \Lambda^{1 / 2}(h, \zeta)
$$

and hence

$$
\begin{aligned}
\Lambda(h, \zeta) / \Lambda\left(h, \zeta^{\prime}\right) & \leqslant \min \left\{\Lambda(h, \zeta),\left(1-\Lambda^{-1 / 2}(h, \zeta)\right)^{-1}\right\} \\
& \leqslant(3+\sqrt{5}) / 2=c_{0} .
\end{aligned}
$$

By (4.39) and (4.40) we get that the constant $K$ in (4.38) is bounded by

$$
K \leqslant c_{0}|p|_{1,0,2,0}<\infty .
$$

Proof of Lemma 4.6.3. Let $v=\left(B_{\eta}^{h}-\operatorname{Re} A_{\eta}^{h}\right) u$. Then

$$
\hat{v}^{h}(\zeta)=(2 \pi)^{-n} \int_{T_{h}^{n}}{\widehat{k^{\prime}}}^{h}\left(h, \zeta, \xi-\xi^{\prime}, \zeta^{\prime}\right) \hat{u}^{h}\left(\zeta^{\prime}\right) d \xi^{\prime}+k(h, \zeta, \infty, \zeta) \hat{u}^{h}(\zeta)
$$

where

$$
2 k\left(h, \zeta, x, \zeta^{\prime}\right)=2 b\left(h, \zeta, x, \zeta^{\prime}\right)-a\left(h, x, \zeta^{\prime}\right)-a^{*}(h, x, \zeta) .
$$

From (4.33) and (4.34) follows

(4.44) $2 k\left(h, \zeta, x, \zeta^{\prime}\right)=-\int_{T_{h}^{n}} p\left(h, x, \zeta^{\prime \prime}\right)\left[\Phi\left(h, \zeta^{\prime}, \zeta^{\prime \prime}\right)-\Phi\left(h, \zeta, \zeta^{\prime \prime}\right)\right]^{2} d \xi^{\prime \prime}$ 
Hence, $k(h, \zeta, \infty, \zeta)=0$ and

$$
\begin{aligned}
2{\widehat{k^{\prime}}}^{h}(h, \zeta, \xi & \left.-\xi^{\prime}, \zeta^{\prime}\right) \\
& =-\int_{T_{h}^{n}}{\widehat{p^{\prime}}}^{h}\left(h, \xi-\xi^{\prime}, \zeta^{\prime \prime}\right)\left[\Phi\left(h, \zeta^{\prime}, \zeta^{\prime \prime}\right)-\Phi\left(h, \zeta, \zeta^{\prime \prime}\right)\right]^{2} d \xi^{\prime \prime}
\end{aligned}
$$

Represent the above integrand as a product of the following two functions:

(4.46) $q\left(h, \chi, \zeta^{\prime \prime}\right)=\Lambda^{2}(h, \chi, 0){\widehat{p^{\prime}}}^{h}\left(h, \chi, \zeta^{\prime \prime}\right) \Lambda^{-1}\left(h, \zeta^{\prime \prime}\right), \quad \chi=\xi-\xi^{\prime}$, and

(4.47) $\Psi\left(h, \zeta, \zeta^{\prime}, \zeta^{\prime \prime}\right)=\Lambda^{-2}\left(h, \zeta^{\prime}-\zeta\right) \Lambda\left(h, \zeta^{\prime \prime}\right)\left[\Phi\left(h, \zeta^{\prime}, \zeta^{\prime \prime}\right)-\Phi\left(h, \zeta, \zeta^{\prime \prime}\right)\right]^{2}$.

In view of (4.42) and (4.45) the norm of the operators

is bounded by

$$
B_{\eta}^{h}-\operatorname{Re} A_{\eta}^{h}: H_{0, n, h} \rightarrow H_{0, \eta, h}
$$

$$
\int_{T_{h}^{n} \sup _{\zeta^{\prime \prime}, h}}\left|q\left(h, \chi, \zeta^{\prime \prime}\right)\right| d \chi \cdot \sup _{\zeta, \zeta^{\prime}, h} \int_{T_{h}^{n}} \Psi\left(h, \zeta, \zeta^{\prime}, \zeta^{\prime \prime}\right) d \xi^{\prime \prime} .
$$

The first integral in (4.48) is bounded by $|p(h, x, \zeta)|_{1,0,1,2}$. The function $\Psi\left(h, \zeta, \zeta^{\prime}, \zeta^{\prime \prime}\right)$ is estimated by

$$
\begin{aligned}
& \Psi\left(h, \zeta, \zeta^{\prime}, \zeta^{\prime \prime}\right) \\
& \quad \leqslant \Lambda\left(h, \zeta^{\prime \prime}\right) \Lambda^{-2}\left(h, \zeta^{\prime}-\zeta\right)\left|\zeta^{\prime}-\zeta\right|^{2}\left(\int_{0}^{1}\left|\dot{\Phi}\left(h, \zeta_{t}, \zeta^{\prime \prime}\right)\right| d t\right)^{2}
\end{aligned}
$$

where $\zeta_{t}=\zeta+t\left(\zeta^{\prime}-\zeta\right)$ and $\dot{\Phi}\left(h, \zeta, \zeta^{\prime \prime}\right)$ is the gradient of $\Phi\left(h, \zeta, \zeta^{\prime \prime}\right)$ with respect to the variable $\xi$. Since the function $\Psi\left(h, \zeta, \zeta^{\prime}, \zeta^{\prime \prime}\right)$ is periodic in $\xi$ and $\xi^{\prime}$, we may assume that $\xi^{\prime}-\xi \in T_{h}^{n}$ and therefore, as in (4.23),

$$
\left|\zeta^{\prime}-\zeta\right| \Lambda^{-1}\left(h, \zeta^{\prime}-\zeta\right) \leqslant \pi / 2
$$

Changing the order of integration in triple integrals and using Schwarz' inequality, we derive from (4.49) and (4.50)

$$
\begin{aligned}
& \int_{T_{h}^{n}} \Psi\left(h, \zeta, \zeta^{\prime}, \zeta^{\prime \prime}\right) d \xi^{\prime \prime} \\
& \quad \leqslant(\pi / 2)^{2}\left[\int_{0}^{1} d t\left(\int_{T_{h}^{n}}\left|\dot{\Phi}\left(h, \zeta_{t}, \zeta^{\prime \prime}\right)\right|^{2} \Lambda\left(h, \zeta^{\prime \prime}\right) d \xi^{\prime \prime}\right)^{1 / 2}\right]^{2}
\end{aligned}
$$

For small $h$

$$
\left|\dot{\Phi}\left(h, \zeta_{t}, \zeta^{\prime \prime}\right)\right|^{2}=\sum_{\alpha \in \mathbf{Z}^{n}}\left|\dot{\varphi}_{h}\left(\zeta_{t}, \xi^{\prime \prime}+2 \pi h^{-1} \alpha, \eta\right)\right|^{2}
$$

and hence

$$
\int_{T_{h}^{n}}\left|\dot{\Phi}\left(h, \zeta_{t}, \zeta^{\prime \prime}\right)\right|^{2} \Lambda\left(h, \zeta^{\prime \prime}\right) d \xi^{\prime \prime}=\int_{R^{n}}\left|\dot{\varphi}_{h}\left(\zeta_{t}, \zeta^{\prime \prime}\right)\right|^{2} \Lambda\left(h, \zeta^{\prime \prime}\right) d \xi^{\prime \prime}
$$

Note that $\dot{\varphi}_{h}\left(\zeta_{t}, \zeta^{\prime \prime}\right) \neq 0$ implies $\left|\zeta_{t}-\zeta^{\prime \prime}\right| \leqslant \Lambda^{1 / 2}\left(h, \zeta_{t}\right)$. In this case, using the inequality $\left|\Lambda\left(h, \zeta_{t}\right)-\Lambda\left(h, \zeta^{\prime \prime}\right)\right| \leqslant\left|\zeta_{t}-\zeta^{\prime \prime}\right| \leqslant \Lambda^{1 / 2}\left(h, \zeta_{t}\right)$, we get

$$
\Lambda\left(h, \zeta^{\prime \prime}\right) / \Lambda\left(h, \zeta_{t}\right) \leqslant 2 \text {, }
$$


and therefore

$$
\int_{R^{n}}\left|\dot{\varphi}_{h}\left(\zeta_{t}, \zeta^{\prime \prime}\right)\right|^{2} \Lambda\left(h, \zeta^{\prime \prime}\right) d \xi^{\prime \prime} \leqslant 2 \int_{R^{n}}\left|\dot{\varphi}_{h}\left(\zeta_{t}, \zeta^{\prime \prime}\right)\right|^{2} \Lambda\left(h, \zeta_{t}\right) d \xi^{\prime \prime}
$$

By direct differentiation one could easily verify that

$$
\begin{aligned}
& \left|\Lambda^{1 / 2}(h, \zeta) D_{\xi_{j}} \varphi_{h}\left(\zeta, \zeta^{\prime \prime}\right)\right| \\
& =\left|\Lambda^{1 / 2}(h, \zeta) D_{\xi_{j}}\left[\Lambda^{-n / 4}(h, \zeta) \varphi\left(\Lambda^{-1 / 2}(h, \zeta)\left(\xi^{\prime \prime}-\xi\right)\right)\right]\right| \\
& \leqslant K \Lambda^{-n / 4}(h, \zeta)\left[\varphi\left(\Lambda^{-1 / 2}(h, \zeta)\left(\xi^{\prime \prime}-\xi\right)\right)\right. \\
& \left.\quad+\sum_{i=1}^{n}\left|\varphi_{i}\left(\Lambda^{-1 / 2}(h, \zeta)\left(\xi^{\prime \prime}-\xi\right)\right)\right|\right],
\end{aligned}
$$

where $\varphi_{i}(\xi)=D_{\xi_{i}} \varphi(\xi)$, so that the right-hand side in (4.53) is bounded by a constant times the integral

$$
\int_{R^{n}}\left[\varphi(\theta)+\sum_{j=1}^{n}\left|\varphi_{j}(\theta)\right|\right]^{2} d \theta<\infty
$$

The theorem is proved.

5. Proof of Estimate (2.16) in the Case of Variable Coefficients. Using the algebra of P.D.O. built in Section 4 we shall modify the proof of estimate (2.16) in subsection 3.3 to be valid for variable coefficients. The different symbols, as $X(\zeta)$, $T(\zeta), M(\zeta), R^{\prime}\left(\zeta^{\prime},|\zeta|\right)$ etc., which appear in Section 3, are, actually, smooth functions of $x=\left(x_{0}, x_{1}, \ldots, x_{n-1}\right)$ and $t$ in a neighborhood $\Omega_{x, t}$ of a point $(x, t)_{0}$. We shall consider $x_{0}$ as a parameter and identify $\left(x_{-}, t\right)$ with the vector $x=$ $\left(x_{1}, x_{2}, \ldots, x_{n-1}, x_{n}\right)$ in Section 4. If $A(x, t, \zeta)$ is some matrix function in Section 3 (appearing there without the variables $x, t$ ), we denote by $A_{\eta}^{h}$ the family of P.D.O. depending on parameters $\eta, h$ and $x_{0}$ with the symbol $\sigma\left(A_{n}^{h}\right)=A\left(x_{0},\left(x_{-}, t\right), h \zeta\right)$. Thus we rewrite problem $(2.11)$ as

$$
\begin{aligned}
& \text { (A) } \tilde{L}_{\eta}^{h}\left(E_{0}\right) u\left(x_{0}\right)=\left(A_{\eta}^{h} E_{0}+B_{\eta}^{h}\right) u\left(x_{0}\right)=h F\left(x_{0}\right), \quad x_{0} \in R_{h}^{+}, \\
& \text {(B) } \tilde{S}_{\eta}^{h} u(0)=g,
\end{aligned}
$$

where $u\left(x_{0}\right), F\left(x_{0}\right), g$ are grid vector-functions depending on $\left(x_{-}, t\right) \in R_{h}^{n}$. To simplify notations we denote the norms $\|\cdot\|_{k, \eta, h}$ by $|\cdot|_{k, \eta}$ if $k \neq 0$ and by $|\cdot|_{\eta}$ if $k=0$, and the scalar products $(\cdot, \cdot)_{0, \eta, h}$ in $H_{0, \eta, h}$ by $(\cdot, \cdot)_{\eta}$. Also replace the norms $\|\cdot\|_{x, t, \eta}$ and scalar product $\langle\cdot, \cdot\rangle_{x, t, \eta}$ in (1.11) by $\|\cdot\|_{\eta}$ and $\langle\cdot, \cdot\rangle_{\eta}$. As shown in Section 2 , we may restrict ourselves to $1 \leqslant \eta \leqslant K_{0} / h$. Finally estimate $(2.16)$ becomes

$$
\eta\|u\|_{\eta}^{2}+|u(0)|_{\eta}^{2} \leqslant K\left(\eta^{-1}\|F\|_{\eta}^{2}+|g|_{\eta}^{2}\right)
$$

Let $\Omega_{x, t} \times \Omega\left(\zeta_{0}\right)$ be a neighborhood of a point $(x, t)_{0}, \zeta_{0}$ in which equivalences (3.22) or (3.26) hold with the matrix-functions $X, T, M_{f}, M_{\infty}$ depending smoothly on $(x, t, \zeta)$, and let $\varphi(x, t, \zeta)$ be a cut-off function which is equal to one in a neighborhood of $(x, t)_{0}, \zeta_{0}$ and has support in $\Omega_{x, t} \times \Omega\left(\zeta_{0}\right)$. We may assume that a 
finite set $J$ of such neighborhoods covers the domain $x_{0} \geqslant 0,\left(x_{-}, t\right) \in R^{n}, \xi \in R^{n}$, $0 \leqslant \eta \leqslant K_{0}$ and the corresponding cut-off functions $\varphi_{j}(x, t, \zeta), j \in J$, form a partition of unity, i.e. $\Sigma_{j \in J} \varphi_{j}(x, t, \zeta)=1$. Let $\Phi_{j, \eta}^{h}$ be a family of P.D.O. with the symbol $\varphi_{j}\left(x_{0}, x_{-}, t, h \zeta\right)$ which belongs to $\tilde{S}_{1,0}^{0,0}$ for any $x_{0} \in R^{+}$. Due to Theorem 4.2a, the grid function $\Phi_{j, \eta}^{h} u \in e^{\eta t} l_{2}(x, t)$ satisfies the equation

$$
\begin{aligned}
& \text { (A) }\left(A_{\eta}^{h} E_{0}+B_{\eta}^{h}\right) \Phi_{j, \eta}^{h} u\left(x_{0}\right)=h \Phi_{j, \eta}^{h} F\left(x_{0}\right)+O(h) u, \\
& \text { (B) } \tilde{S}_{\eta}^{h} \Phi_{j, \eta}^{h} u(0)=\Phi_{j, \eta}^{h} g+O(h) u(0),
\end{aligned}
$$

where $O(h)$ in (A) and (B) are correspondingly operators in $e^{\eta t} l_{2}(x, t)$ and $e^{\eta t} l_{2}\left(x_{-}, t\right)$ with the norm bounded by $K h$. Suppose that for the system in (5.3) with the right-hand side denoted by $h F$ and $g$ correspondingly we have the estimate

$$
\eta\left\|\Phi_{j, \eta}^{h} u\right\|_{\eta}^{2}+\left|\Phi_{j, \eta}^{h} u(0)\right|^{2} \leqslant K\left(\eta^{-1}\|F\|_{\eta}^{2}+|g|_{\eta}^{2}+\|u\|_{\eta}^{2}+\eta^{-1}|u(0)|_{\eta}^{2}\right) .
$$

Then, $u=\Sigma_{j \in J} \Phi_{j, \eta}^{h} u$ and $F, g$ in (5.1) satisfy the estimate

$$
\begin{gathered}
\eta\|u\|_{\eta}^{2}+|u(0)|_{\eta}^{2} \leqslant|J|^{2} \sum_{j \in J}\left(\eta\left\|\Phi_{j, \eta}^{h} u\right\|_{\eta}^{2}+\left|\Phi_{j, \eta}^{h} u(0)\right|_{\eta}^{2}\right) \\
\leqslant K\left[\eta^{-1} \sum_{j}\left\|\Phi_{j, \eta}^{h} F\right\|_{\eta}^{2}+\sum_{j}\left|\Phi_{j, \eta}^{h} g\right|_{\eta}^{2}+\left(\eta^{-1}+1\right)\|u\|_{\eta}^{2}\right. \\
\left.+\left(\eta^{-1}+h^{2}\right)|u(0)|_{\eta}^{2}\right] \\
\leqslant K\left[\eta^{-1}\|F\|_{\eta}^{2}+|g|_{\eta}^{2}+\|u\|_{\eta}^{2}+\eta^{-1}|u(0)|_{\eta}^{2}\right],
\end{gathered}
$$

which for large $\eta$ is equivalent to (5.2).

Thus we consider the equations

$$
\begin{aligned}
& \text { (A) }\left(A_{\eta}^{h} E_{0}+B_{\eta}^{h}\right) \Phi_{\eta}^{h} u=h F, \\
& \text { (B) } \tilde{S}_{\eta}^{h} \Phi_{\eta}^{h} u(0)=g .
\end{aligned}
$$

If the neighborhood $\Omega_{x, t}$ does not intersect the boundary, so does the support of the grid function $\Phi_{\eta}^{h} u$, and estimate (5.4) follows from the well-posedness of the Cauchy problem.

Now, as in subsection 3.3, we restrict ourselves to a more difficult case when $\Omega_{x, t}$ is a neighborhood of a boundary point $\left(0,\left(x_{-}\right)_{0}, t_{0}\right)$ and $\Omega\left(\zeta_{0}\right)$ corresponds to $\zeta_{0}=0$. We may assume that equivalence (3.26) holds on supp $\varphi(x, t, \zeta)$ and that the matrix-functions $X, X^{-1}, T, T^{-1}, M_{f}, M_{\infty}$ vanish outside some open set which includes supp $\varphi(x, t, \zeta)$. Denote

$$
v=\left(X^{-1}\right)_{\eta}^{h} u, \quad G=\left(T^{-1}\right)_{\eta}^{h} F .
$$

We assume that $v$ and $G$ are partitioned as in (3.74). Note that the symbols $X(x, t, h \zeta)$ etc. belong to $\tilde{S}_{1,0}^{0,0}$. Since $X \varphi X^{-1}=\varphi$, we have

$$
X_{\eta}^{h} \Phi_{\eta}^{h} v=\Phi_{\eta}^{h} u+O(h) u
$$

and

$$
\left\{\begin{array}{l}
\left(T^{-1}\right)_{\eta}^{h}\left(A_{\eta}^{h} E_{0}+B_{\eta}^{h}\right) X_{\eta}^{h} \Phi_{\eta}^{h} v=h G+O(h) u, \\
\tilde{S}_{\eta}^{h} X_{\eta}^{h} \Phi_{\eta}^{h} v(0)=g+O(h) u(0) .
\end{array}\right.
$$


Then, equivalence (3.26) on the support of $\varphi(x, t, \zeta)$ implies
(A) $\left(E_{0}-M_{f, \eta}^{h}\right) \Phi_{\eta}^{h} v_{f}=h G_{f}+O(h) u$,
(B) $\left(-M_{\infty, \eta}^{h} E_{0}+I\right) \Phi_{\eta}^{h} v_{\infty}=h G_{\infty}+O(h) u$,
(C) $\tilde{S}_{\eta}^{h} X_{\eta}^{h} \Phi_{\eta}^{h} v(0)=g+O(h) u(0)$.

Suppose that for the system in (5.8) without the terms $O(h) u$ we have the estimate

$$
\eta\left\|\Phi_{\eta}^{h} v\right\|_{\eta}^{2}+\left|\Phi_{\eta}^{h} v(0)\right|_{\eta}^{2} \leqslant K\left(\eta^{-1}\|G\|_{\eta}^{2}+|g|_{\eta}^{2}+\|v\|_{\eta}^{2}+\eta^{-1}|v(0)|_{\eta}^{2}\right) .
$$

Then, by (5.6) and (5.7), we get for $\Phi_{\eta}^{h} u$ the desired estimate (5.4). We define the symbols $R_{0}(x, t, \zeta), \quad R_{\infty}(x, t, \zeta), \quad R^{\prime}(x, t, \zeta)=R^{\prime}\left(x, t, \zeta^{\prime},|\zeta|\right), \quad R_{f}(x, t, \zeta)$ and $R(x, t, \zeta)$ as in (3.76). Note that $R_{0}(x, t, h \zeta)$ and $R_{\infty}(x, t, h \zeta)$ belong to $\tilde{S}_{1,0}^{0,0}$, and $R^{\prime}(x, t, h \zeta) \in S_{1,0}^{0,0}$ for $\eta \geqslant 1$. Multiply Eqs. (5.8)(A) and (5.8)(B) (without the terms $O(h) u)$ by $R_{f, \eta}^{h}\left(-E_{0}-M_{f, \eta}^{h}\right) \Phi_{\eta}^{h} v_{f}$ and $R_{\infty, \eta}^{h}\left(M_{\infty, \eta}^{h} E_{0}+I\right) \Phi_{\eta}^{h} v_{\infty}$ in the sense of the scalar product in $e^{\eta t} l_{2}(x, t)$, and compare real parts. Instead of (3.77) and (3.78) we obtain

$$
\begin{gathered}
\operatorname{Re}\left\{\left\langle\left[\left(M_{f, \eta}^{h}\right) * R_{f, \eta}^{h} M_{f, \eta}^{h}-R_{f, \eta}^{h}\right] \Phi_{\eta}^{h} v_{f}, \Phi_{\eta}^{h} v_{f}\right\rangle_{\eta}\right. \\
\left.+h\left(R_{f, \eta}^{h} \Phi_{\eta}^{h} v_{f}(0), \Phi_{\eta}^{h} v_{f}(0)\right)_{\eta}+\left\langle\Delta R_{f, \eta}^{h} E_{0} \Phi_{\eta}^{h} v_{f}, E_{0} \Phi_{\eta}^{h} v_{f}\right\rangle_{\eta}\right\} \\
=-\operatorname{Re}\left\langle\left[\left(R_{f, \eta}^{h}\right) * M_{f, \eta}^{h}+R_{f, \eta}^{h} E_{0}\right] \Phi_{\eta}^{h} v_{f}, h G_{f}\right\rangle_{\eta}
\end{gathered}
$$

and a similar equality for $\Phi_{\eta}^{h} v_{\infty}$. Here $\Delta R_{f, \eta}^{h}$ has the symbol $R_{f}\left(x_{0}+h, x_{-}, t, h \zeta\right)$ $-R_{f}\left(x_{0}, x_{-}, t, h \zeta\right)$ and therefore the norm of $\Delta R_{f, \eta}^{h}: H_{0, \eta, h} \rightarrow H_{0, \eta, h}$ is bounded by $K h$. Consider first the term

$$
\operatorname{Re}\left\langle\left[\left(M_{0, \eta}^{h}\right) * R_{0, \eta}^{h} M_{0, \eta}^{h}-R_{0, \eta}^{h}\right] \Phi_{\eta}^{h} v_{0}, \Phi_{\eta}^{h} v_{0}\right\rangle_{\eta} .
$$

Since the symbols $p_{0}(x, t, h \zeta)=M_{0}^{*}(x, t, h \zeta) R_{0} M_{0}(x, t, h \zeta)-R_{0}$ and $\varphi(x, t, \zeta)$ satisfy the conditions of Theorem $4.5 \mathrm{a}$, the above term is bounded from below by $\delta / 2\left\|\Phi_{\eta}^{h} v_{0}\right\|_{\eta}^{2}-K h\left\|v_{0}\right\|_{\eta}^{2}$. The symbol

$$
p_{1}(x, t, \zeta)=M_{1}^{*} R^{\prime} M_{1}-R^{\prime}=\left(M_{1}-I\right)^{*} R^{\prime} M_{1}+R^{\prime}\left(M_{1}-I\right)
$$

belongs to $S_{1,0}^{1}$, and for $(x, t, \zeta) \in \operatorname{supp} \varphi(x, t, \zeta)$ estimate (3.68) holds. Note also that the symbol $\left(M_{1}(x, t, h \zeta)-I\right) / h$ belongs to $S_{1,0}^{1,0}$ and therefore the operators $\left(M_{1, \eta}^{h}-I\right) / h: H_{k+1, \eta, h} \rightarrow H_{k, \eta, h}$ are uniformly bounded. Introduce the symbol

$$
p_{2}(x, t, h \zeta)=\left(h^{-1} p_{1}(x, t, h \zeta)-\delta \eta I / 2\right) \varphi^{2}(x, t, h \zeta),
$$

which for $\eta \geqslant 1$ belongs to $S_{1,0}^{1,0}$ and satisfies the conditions of Theorem 4.6. Using Theorems 4.1, 4.2, 4.3 and 4.6, we get

$$
\begin{aligned}
\operatorname{Re}\left\langle\left[\left(M_{1, \eta}^{h}\right) *\right.\right. & \left.\left.R_{\eta}^{\prime h} M_{1, \eta}^{h}-R_{\eta}^{\prime h}\right] \Phi_{\eta}^{h} v_{1}, \Phi_{\eta}^{h} v_{1}\right\rangle_{\eta} \\
& =\operatorname{Re}\left\langle P_{1, \eta}^{h} \Phi_{\eta}^{h} v_{1}, \Phi_{\eta}^{h} v_{1}\right\rangle_{\eta}+O(h)\left\|\Phi_{\eta}^{h} v_{1}\right\|_{\eta}^{2} \\
& =h \cdot \operatorname{Re}\left\langle P_{2, \eta}^{h} v_{1}, v_{1}\right\rangle_{\eta}+\delta \eta h / 2\left\|\Phi_{\eta}^{h} v_{1}\right\|_{\eta}^{2}+O(h)\left\|v_{1}\right\|_{\eta}^{2} \\
& \geqslant \delta \eta h / 2\left\|\Phi_{\eta}^{h} v_{1}\right\|_{\eta}^{2}-K h\left\|v_{1}\right\|_{\eta}^{2} .
\end{aligned}
$$

The right-hand side in (5.10) is bounded from above by

$$
K\left(h^{2}\left\|G_{0}\right\|_{\eta}^{2}+h \eta^{-1}\left\|G_{1}\right\|_{\eta}^{2}\right)+\delta^{\prime}\left(\left\|\Phi_{\eta}^{h} v_{0}\right\|_{\eta}^{2}+\eta h\left\|\Phi_{\eta}^{h} v_{1}\right\|_{\eta}^{2}\right)
$$


with $\delta^{\prime}<\delta / 2$. Collecting all the above estimates, we obtain

$$
\begin{aligned}
\delta\left(\left\|\Phi_{\eta}^{h} v_{0}\right\|_{\eta}^{2}+\eta h\left\|\Phi_{\eta}^{h} v_{1}\right\|_{\eta}^{2}\right)+h\left(R_{f, \eta}^{h} \Phi_{\eta}^{h} v_{f}(0), \Phi_{\eta}^{h} v_{f}(0)\right)_{\eta} \\
\quad \leqslant K\left(h^{2}\left\|G_{0}\right\|_{\eta}^{2}+h \eta^{-1}\left\|G_{1}\right\|_{\eta}^{2}+h\left\|v_{f}\right\|_{\eta}^{2}\right) .
\end{aligned}
$$

Since $\eta h \leqslant K_{0}$, the last estimate implies

$$
\delta \eta\left\|\Phi_{\eta}^{h} v_{f}\right\|_{\eta}^{2}+\left(R_{f, \eta}^{h} \Phi_{\eta}^{h} v_{f}(0), \Phi_{\eta}^{h} v_{f}(0)\right)_{\eta} \leqslant K\left(\eta^{-1}\left\|G_{f}\right\|_{\eta}^{2}+\left\|v_{f}\right\|_{\eta}^{2}\right) .
$$

In a similar way we get for $\Phi_{\eta}^{h} v_{\infty}$ instead of (3.81) the estimate

$$
\delta\left\|\Phi_{\eta}^{h} v_{\infty}\right\|_{\eta}^{2}+h\left(R_{\infty, \eta}^{h} \Phi_{\eta}^{h} v_{\infty}(0), \Phi_{\eta}^{h} v_{\infty}(0)\right)_{\eta} \leqslant K\left(h^{2}\left\|G_{\infty}\right\|_{\eta}^{2}+h\left\|v_{\infty}\right\|_{\eta}^{2}\right)
$$

and thus

$$
\eta\left\|\Phi_{\eta}^{h} v\right\|_{\eta}^{2}+\left(R_{\eta}^{h} \Phi_{\eta}^{h} v(0), \Phi_{\eta}^{h} v(0)\right)_{\eta} \leqslant K\left(\eta^{-1}\|G\|_{\eta}^{2}+\|v\|_{\eta}^{2}\right) .
$$

The symbol

$$
q(x, t, \zeta)=R+K X^{*} \tilde{S^{*}} \tilde{S} X
$$

in (3.84) satisfies the conditions of Theorem 4.5. Therefore

$$
\begin{aligned}
\left(R_{\eta}^{h} \Phi_{\eta}^{h} v(0), \Phi_{\eta}^{h} v(0)\right)_{\eta} & =\left(Q_{\eta}^{h} \Phi_{\eta}^{h} v(0), \Phi_{\eta}^{h} v(0)\right)_{\eta}-K|g|_{\eta}^{2}+O\left(|v(0)|_{-1 / 2, \eta}^{2}\right) \\
& \geqslant \delta\left\|\Phi_{\eta}^{h} v(0)\right\|^{2}-K|g|_{\eta}^{2}-K_{1}|v(0)|_{-1 / 2, \eta}^{2} .
\end{aligned}
$$

However,

and estimate (5.9) follows. Q.E.D.

$$
|v(0)|_{-1 / 2, \eta}^{2} \leqslant \eta^{-1}|v(0)|_{\eta}^{2}
$$

\section{Proof of Theorems 1.1 and 1.2.}

Proof of Theorem 1.1. Sufficiency of conditions (a), (b) and (c). In view of Lemma 2.2, we start with the linearized problem (2.20). We consider the general case of variable coefficients and use the algebra of P.D.O. from Section 4 with the symbols $a\left(h, x, \xi_{-}\right)=a\left(h, x_{0}, x_{-}, \xi_{-}\right) \in \tilde{S}_{1,0}^{0,0}$ and $\eta=0$. Here $x_{0} \in R^{+}$is a parameter, $x_{-} \in R^{n-1}$ and $\xi_{-} \in R^{n-1}$ is the dual variable. The corresponding family of P.D.O. is denoted by $A^{h}$ (but depends also on $x_{0}$ ) and acts in the space $H_{0,0, h}=l_{2}\left(x_{-}\right)$. To shorten the notations we also remove the signs ${ }^{\tilde{a}}$ and $(0)$ from $\tilde{L}^{(0)}$ and $\tilde{S}^{(0)}$, omit the dummy variable $t$ and rewrite (2.20) as

$$
\begin{aligned}
& \text { (A) } L^{h}\left(E_{0}\right) u\left(x_{0}\right)=\left(A^{h} E_{0}+B^{h}\right) u\left(x_{0}\right)=F\left(x_{0}\right), \quad x_{0} \in R_{h}^{+}, \\
& \text {(B) } S^{h} u(0)=g,
\end{aligned}
$$

where $A^{h}, B^{h}$ and $S^{h}$ have correspondingly the symbols $A\left(x, h \xi_{-}\right), B\left(x, h \xi_{-}\right)$and $S\left(x, h \xi_{-}\right)$. Condition (a) in Theorem 1.1 implies that the characteristic equation

$$
\operatorname{det}\left(A\left(x, h \xi_{-}\right) \kappa+B\left(x, h \xi_{-}\right)\right)=0
$$

has no solutions on the unit circle $|\kappa|=1$. Therefore, in a neighborhood $\Omega_{x} \times \Omega_{h \xi_{-}}$ of any point $(x)_{0},\left(h \xi_{-}\right)_{0}$ the pencil $A\left(x, h \xi_{-}\right) \kappa+B\left(x, h \xi_{-}\right)$may be brought to a block form

$$
\begin{aligned}
\left(A\left(x, h \xi_{-}\right) \kappa+\right. & \left.B\left(x, h \xi_{-}\right)\right) X\left(x, h \xi_{-}\right) \\
& =T\left(x, h \xi_{-}\right)\left(\begin{array}{cc}
\kappa I-M_{0}\left(x, h \xi_{-}\right) & 0 \\
0 & -\kappa M_{\infty}\left(x, h \xi_{-}\right)+I
\end{array}\right),
\end{aligned}
$$


where the matrix-functions $M_{0}$ and $M_{\infty}$ are as in (3.21), and the symbols $X, T, M_{0}$ and $M_{\infty}$ belong to $\tilde{S}_{1,0}^{0,0}$. According to condition (b) the matrix

$$
N\left(x, h \xi_{-}\right)=S\left(x, h \xi_{-}\right) X_{0}\left(x, h \xi_{-}\right)
$$

is of order $l_{0} \times l_{0}$, and condition (c) actually states that

$$
\operatorname{det} N\left(x, h \xi_{-}\right) \neq 0 \text { for any } h \xi_{-} \text {and any } x=\left(0, x_{-}\right) \text {. }
$$

Condition (6.3) is an analog of (UKC) for the initial-boundary value problem. We define symmetrizers $R_{0}=-c I$ and $R_{\infty}=I$ as in (3.76) with $R=\operatorname{diag}\left(R_{0}, R_{\infty}\right)$. Then, for small $c$, we have as in (3.84)

$$
R+K(S X) * S X \geqslant \delta I \text {. }
$$

Now we repeat those parts of the proof in Section 5 which are related to the blocks $M_{0}$ and $M_{\infty}$ and get easily the required estimate (1.16). To prove the existence of solution in (6.1) we look at the pair $\mathcal{L}=\left(L^{h}\left(E_{0}\right), S^{h}\right)$ as an operator from $l_{2}(x)$ to the space $H=\left\{(F, g) \mid F \in l_{2}(x), g \in l_{2}\left(x_{-}\right)\right\}$with the norm $\|(F, g)\|^{2}=\|F\|_{x}^{2}$ $+h\|g\|_{x_{-}}^{2}$ and consider the adjoint problem

$$
\begin{aligned}
& \text { (A) }\left(E_{0} B^{h^{*}}+A^{h^{*}}\right) F\left(x_{0}\right)=u\left(x_{0}+h\right), \quad x_{0} \in R_{h}^{+}, \\
& \text {(B) } B^{h^{*}} F(0)+S^{h^{*}} g=u(0) .
\end{aligned}
$$

The pencil of matrices $B^{*}\left(x, h \xi_{-}\right) \kappa+A^{*}\left(x, h \xi_{-}\right)$is equivalent to a block form of the same type as in (6.2) with $X$ and $T$ replaced by $\tilde{X}=\left(\tilde{X}_{0}, \tilde{X}_{\infty}\right)=\left(T^{*}\right)^{-1}$ and $\tilde{T}=\left(X^{*}\right)^{-1}$. Note that the operator $\mathcal{E}$ with coefficients frozen at some boundary point is an isomorphism, since one can find the solution of (6.1) by applying the Fourier transform and equivalence (6.2). Therefore the adjoint operator $\mathcal{L}^{*}$ with frozen coefficients is an isomorphism too. The last implies that the matrix

$$
\tilde{N}\left(x, h \xi_{-}\right)=\left[B^{*}\left(x, h \xi_{-}\right) \tilde{X}_{0}\left(x, h \xi_{-}\right), S^{*}\left(x, h \xi_{-}\right)\right]
$$

is nonsingular. The system in (6.5) is of a slightly different type than the one in (6.1). However, we could extend the grid function $g$ to $x_{0}>0$ and add to (6.5)(A) the equations

$$
\left(E_{0} I+0\right) g\left(x_{0}\right)=0, \quad x_{0} \in R_{h}^{+} .
$$

Then problem (6.5) becomes of the same type as (6.1), and we get instead of (1.16) the estimate

$$
\|F\|_{x}^{2}+h\|g\|_{x_{-}}^{2} \leqslant K\|u\|_{x}^{2}
$$

which proves the sufficiency part of the theorem.

Necessity. Suppose that condition (a) is violated at some point $(x)_{0}, t_{0}, \xi$ and vector $u_{0}$ belongs to the kernel of $L^{(0)}\left(0,(x)_{0}, t_{0}, e^{i \xi}\right)$. Let $\varphi_{\varepsilon}(x)$ be a cut-off function with support in the ball $\left|x-(x)_{0}\right|<\varepsilon$ and in the half-plane $x_{0}>0$. Then, for small $\varepsilon$ and $h$, the function $u(x)=\varphi_{\varepsilon}(x) u_{0} \exp (i \xi x / h)$ violates estimate (1.16) with $g=0$. Further, if problem (1.19) with the coefficients frozen at some boundary point $\left((x)_{0}, t_{0}\right)$ and some $\xi_{-}$has a nontrivial solution $u_{0}\left(x_{0}\right)$, we build a grid function

$$
u(x)=\varphi_{\varepsilon}(x) u_{0}\left(x_{0} / h\right) \exp \left(i x_{-} \xi_{-} / h\right),
$$

where this time $\varphi_{\varepsilon}\left((x)_{0}\right)=1$. Again, for small $\varepsilon$ and $h$ the function $u(x)$ violates estimate (1.16). Condition (c) implies that the boundary condition $(1.15)(\mathrm{B})$ is not 
underdetermined. If it is overdetermined, then the boundary condition (6.5)(B) would be underdetermined. The last contradicts estimate (6.6) and, thus, the assumption that the operator $\mathcal{L}$ is an isomorphism.

Proof of Theorem 1.2. We consider the Cauchy problem

$$
\begin{array}{ll}
L u(x, t)=h F(x, t), \quad(x, t) \in R_{h}^{n} \times R_{h}^{+}, t \geqslant \sigma_{0} h, \\
u(x, \sigma h)=f_{\sigma}(x), & x \in R_{h}^{n}, \sigma=0,1, \ldots, \sigma_{0}-1,
\end{array}
$$

where

$$
L=\sum_{\sigma=0}^{-\sigma_{0}} L^{(\sigma)} E_{n}^{\sigma}, \quad L^{(\sigma)}=\sum_{\nu} \sum_{\mu} L_{\nu, \mu, \sigma}(x, t) E_{0}^{\nu} E_{-}^{\mu} .
$$

Our objective is to prove the estimate

$$
\begin{aligned}
\left\|u(x, T) e^{-\eta T}\right\|_{x}^{2}+\eta h & \sum_{t=0}^{T}\left\|u(x, t) e^{-\eta t}\right\|_{x}^{2} \\
& \leqslant K\left(\sum_{\sigma=0}^{\sigma_{0}-1}\left\|f_{\sigma}(x)\right\|_{x}^{2}+\eta^{-1} h \sum_{t=0}^{T}\left\|F(x, t) e^{-\eta t}\right\|_{x}^{2}\right)
\end{aligned}
$$

for $\eta>\eta_{0} \geqslant 0,0<h \leqslant h_{0}$ and $\eta h \leqslant K_{0}$. Here the sum $\Sigma_{t=0}^{T}$ is taken over the grid points in the interval $[0, T=h N]$. Estimate (6.8) obviously implies estimate (1.21). In return, estimate (6.8) follows from (1.21) by the Duhamel principle and, thus, is equivalent to (1.21).

We treat problem (6.7) in the same way as the boundary value problem (1.8). First, linearize problem (6.7) with respect to $t$. As in (2.8) we introduce difference operators

$$
\begin{aligned}
& A=\operatorname{diag}\left(I, \ldots, I, L^{(0)}\right), \\
& B=\left(\begin{array}{ccccc}
0 & -I & 0 & \cdots & 0 \\
0 & 0 & -I & \cdots & 0 \\
\vdots & & & & \vdots \\
L^{\left(-\sigma_{0}\right)} & L^{\left(-\sigma_{0}+1\right)} & & \cdots & -I \\
(-1)
\end{array}\right)
\end{aligned}
$$

and

$$
\tilde{L}\left(E_{n}\right)=A E_{n}+B .
$$

To distinguish between operators and symbols, we denote the operators by $A^{h}, B^{h}$ and $\tilde{L}^{h}\left(E_{n}\right)$ and their symbols by $A(x, t, h \xi), B(x, t, h \xi)$ and $\tilde{L}\left(x, t, h \xi, E_{n}\right)$, correspondingly. We consider $t$ as a parameter and use the algebra of P.D.O. from Section 4 with $\eta=0$. Operator $\tilde{L}^{h}\left(E_{n}\right)$ is related to $L\left(x, t, E_{x}, E_{t}\right)$ by an equivalence analogous to (2.12). Then, as in Lemma 2.1, we get that estimate (6.8) for problem (6.7) is equivalent to the estimate

$$
\begin{aligned}
\left\|u(x, T) e^{-\eta T}\right\|_{x}^{2} & +\eta h \sum_{t=0}^{T}\left\|u(x, t) e^{-\eta t}\right\|_{x}^{2} \\
& \leqslant K\left(\|f(x)\|_{x}^{2}+\eta^{-1} h \sum_{t=0}^{T}\left\|F(x, t) e^{-\eta t}\right\|_{x}^{2}\right)
\end{aligned}
$$


for the problem

$$
\begin{aligned}
\tilde{L}^{h}\left(E_{n}\right) u(x, t) & =h F(x, t), & & (x, t) \in R_{h}^{n} \times R_{h}^{+}, \\
u(x, 0) & =f(x), & & x \in R_{h}^{n} .
\end{aligned}
$$

In view of condition (1.22), for $h \xi$ apart from zero we have a local equivalence

$$
\begin{aligned}
\tilde{L}(x, t, h \xi, z) X(x, t, h \xi)= & T(x, t, h \xi)\left(z I-M_{0}(x, t, h \xi)\right), \\
& \quad \text { where } M_{0}^{*}(x, t, h \xi) M_{0}(x, t, h \xi) \leqslant(1-\varepsilon) I .
\end{aligned}
$$

For $h \xi$ in the neighborhood of zero and $(x, t)$ near $(x)_{0}, t_{0}$ we have like in (3.26)

$$
\begin{aligned}
\tilde{L}(x, t, h \xi, z) & X(x, t, h \xi) \\
& =T(x, t, h \xi) \operatorname{diag}\left(z I-M_{0}(x, t, h \xi), z I-M_{1}(x, t, h \xi)\right),
\end{aligned}
$$

where $M_{1}(x, t, 0)=I$. Introduce conical coordinates

$$
r=|h \xi|, \quad \xi^{\prime}=\xi /|\xi|, \quad z^{\prime}=(z-1) / r
$$

and the matrices

$$
\begin{aligned}
L^{\prime}\left(x, t, \xi^{\prime}, z^{\prime}, r\right) & =L\left(x, t, e^{i \xi^{\prime} r}, 1+z^{\prime} r\right) / r, \\
M^{\prime}\left(x, t, \xi^{\prime}, r\right) & =\left(M_{1}\left(x, t, \xi^{\prime} r\right)-I\right) / r .
\end{aligned}
$$

Then, as in (3.29), one can show that

$$
M^{\prime}\left(x, t, \xi^{\prime}, 0\right)=-i \sum_{j=0}^{n-1} A_{j}(x, t) \xi_{j}^{\prime} .
$$

By strict hyperbolicity, the matrix-function $M^{\prime}\left(x, t, \xi^{\prime}, r\right)$ is locally diagonalizable, i.e. for $\left(\xi^{\prime}, r\right)$ in a neighborhood of $\left(\left(\xi^{\prime}\right)_{0}, 0\right)$ there exists a smooth matrix-function $X^{\prime}=X^{\prime}\left(x, t, \xi^{\prime}, r\right)$ such that

$$
\left(X^{\prime}\right)^{-1} M^{\prime} X^{\prime}=\operatorname{diag}\left(\lambda_{1}^{\prime}, \lambda_{2}^{\prime}, \ldots, \lambda_{k}^{\prime}\right),
$$

where $\lambda_{j}^{\prime}=\lambda_{j}^{\prime}\left(x, t, \xi^{\prime}, r\right), j=1,2, \ldots, k$, are smooth functions. Again, condition (1.22) implies that

$$
\left|1+r \lambda_{j}^{\prime}\left(x, t, \xi^{\prime}, r\right)\right| \leqslant 1 .
$$

We define a local symmetrizer $R^{\prime}=\left[X^{\prime}\left(X^{\prime}\right)^{*}\right]^{-1}$ and use partition of unity in variables $\xi^{\prime}$ to build a global symmetrizer $R^{\prime}(x, t, \xi h)$ which is defined for $0<|\xi h|<\varepsilon$ with $R^{\prime}(x, t, \xi)$ belonging to $S_{1,0}^{0}$. The symbol $R^{\prime}(x, t, \xi h)$ is positive definite and

$$
R^{\prime}-M_{1}^{*} R^{\prime} M_{1} \geqslant 0
$$

for $0<|\xi h|<\varepsilon$ and $(x, t)$ in a neighborhood of $\left((x)_{0}, t_{0}\right)$. We can redefine the symbol $R^{\prime}(x, t, \xi h)$ for $|\xi|<1$ so that a new symbol $R^{\prime}(h, x, t, \xi)$ belongs to $S_{1,0}^{0,0}$, is Hermitian and positive definite, and estimate (6.19) holds for $|\xi| \geqslant 1$. Let $\varphi(x, t, \xi h)$ be a cut-off function with support in a small neighborhood of the point $(x)_{0}, t_{0}$, $\xi h=0$, and $\Phi^{h}$ the corresponding family of P.D.O. As in the proof of Theorem 4.5 one can find a family $R_{1}^{h}$ of P.D.O. such that

(a) $R_{1}^{h}: H_{k, h} \rightarrow H_{k, h}$ and $\left(R_{1}^{h}-R^{\prime h}\right) \Phi^{h}: H_{k, h} \rightarrow H_{k+1, h}$ are uniformly bounded,

(b) $R_{1}^{h}$ is selfadjoint and $R_{1}^{h} \geqslant \delta I$. 
The symbol

$$
\begin{aligned}
p_{1}(h, x, t, \xi) & =h^{-1}\left(R^{\prime}-M_{1}^{*} R^{\prime} M_{1}\right) \varphi^{2} \\
& =\left[h^{-1}\left(I-M_{1}\right)^{*} R^{\prime} M_{1}+R^{\prime}\left(I-M_{1}\right) h^{-1}\right] \varphi^{2}
\end{aligned}
$$

belongs to $S_{1,0}^{1,0}$ and satisfies for $|\xi| \geqslant 1$ the conditions of Theorem 4.6. Therefore

$$
\operatorname{Re}\left(P_{1}^{h} u, u\right)_{0, h} \geqslant-K\|u\|_{0, h}^{2},
$$

and because of (6.20)(a) also

$$
\operatorname{Re}\left(\left[R_{1}^{h}-\left(M_{1}^{h}\right) * R_{1}^{h} M_{1}^{h}\right] \Phi^{h} u, \Phi^{h} u\right)_{0, h} \geqslant-K h\|u\|_{0, h}^{2} .
$$

Now we are ready to prove estimate (6.10). We localize problem (6.11) and then change the variables as in (5.6). Thus we arrive at the problem

$$
\begin{gathered}
\left(E_{n}-M^{h}\right) \Phi^{h} v=\left[E_{n}-\operatorname{diag}\left(M_{0}^{h}, M_{1}^{h}\right)\right] \Phi^{h} v=h G, \quad t \in R_{h}^{+}, \\
\Phi^{h} v=f, \quad t=0 .
\end{gathered}
$$

Instead of (5.9), now we have to prove the local estimate

$$
\begin{aligned}
& \left\|\left(\Phi^{h} v\right)(x, T) e^{-\eta T}\right\|_{x}^{2}+\eta h \sum_{t=0}^{T}\left\|\left(\Phi^{h} v\right)(x, t) e^{-\eta t}\right\|_{x}^{2} \\
& \leqslant K\left(\|f\|_{x}^{2}+\eta^{-1} h \sum_{t=0}^{T}\left\|F(x, t) e^{-\eta t}\right\|_{x}^{2}+h \sum_{t=0}^{T}\left\|v(x, t) e^{-\eta t}\right\|_{x}^{2}\right) .
\end{aligned}
$$

Define $R^{h}=\operatorname{diag}\left(I, R_{1}^{h}\right)$ and multiply the system in (6.22) by $e^{-2 \eta t} R^{h}\left(E_{n}+M^{h}\right) \Phi^{h} v$ in the sense of the scalar product in $l_{2}(x)=H_{0, h}$. Then take sum in $t$ for $t \in R_{h}^{+}$, $0 \leqslant t \leqslant T-h$, and compare real parts. We get

$$
\begin{aligned}
& \left(R^{h} \Phi^{h} e^{-\eta T} v(x, T), \Phi^{h} e^{-\eta T} v(x, T)\right)-\left(R^{h} \Phi^{h} v(x, 0), \Phi^{h} v(x, 0)\right) \\
& \quad+\sum_{t=0}^{T-h} \operatorname{Re}\left(\left[R^{h}-M^{h^{*}} R^{h} M^{h}\right] \Phi^{h} e^{-\eta t} v(x, t), \Phi^{h} e^{-\eta t} v(x, t)\right) \\
& \quad+\sum_{t=h}^{T} \operatorname{Re}\left(\left[\left(e^{2 \eta h}-1\right) R^{h}-e^{2 \eta h} \Delta R^{h}\right] \Phi^{h} e^{-\eta t} v(x, t), \Phi^{h} e^{-\eta t} v(x, t)\right) \\
& \quad=\sum_{t=0}^{T-h} \operatorname{Re}\left(e^{-\eta t} R^{h}\left(E_{n}+M^{h}\right) \Phi^{h} v(x, t), h e^{-\eta t} F(x, t)\right) .
\end{aligned}
$$

The first term in (6.24) is bounded from below by $\delta\left\|\Phi^{h} v(x, T) e^{-\eta T}\right\|_{x}^{2}$, the second from above by $K\|f\|_{x}^{2}$. Because of (6.21) and Theorem 4.5a (for the operator $\left.\left(I-M_{0}^{h^{*}} M_{0}^{h}\right)\right)$, the sum $\Sigma_{t=0}^{T-h}$ in the left-hand side of (6.24) is bounded from below by $-K h \Sigma_{t=0}^{T-h}\left\|e^{-\eta t} v(x, t)\right\|_{x}^{2}$. Since $\eta h<K_{0}$, the term

$$
e^{2 \eta h} \Delta R^{h}=e^{2 \eta h}\left(R^{h}(t)-R^{h}(t-h)\right)
$$

is bounded in the norm of $l_{2}(x)$ by $K h$. Therefore

$$
\begin{aligned}
\left(\left[\left(e^{2 \eta h}-1\right) R^{h}-e^{2 \eta h} \Delta R^{h}\right] \Phi^{h} e^{-\eta t} v(x, t), \Phi^{h} e^{-\eta t} v(x, t)\right) & \\
& \geqslant(\delta \eta h-K h)\left\|\Phi^{h} e^{-\eta t} v(x, t)\right\|_{x}^{2} .
\end{aligned}
$$


The right-hand side in (6.24) is estimated from above by

$$
\sum_{t=0}^{T}\left(\delta^{\prime} \eta h\left\|e^{-\eta t} \Phi^{h} v(x, t)\right\|_{x}^{2}+K \eta^{-1} h\left\|e^{-\eta t} F(x, t)\right\|_{x}^{2}\right) .
$$

Combining all the above estimates and choosing $\delta^{\prime}<\delta / 2$, we arrive at estimate (6.23). Q.E.D.

Department of Mathematics

University of California

Los Angeles, California 90024

1. M. S. Agranovich, "Theorem of matrices depending on parameters and its applications to hyperbolic systems," Functional Anal. Appl., v. 6, 1972, pp. 85-93. (Russian)

2. L. S. FranK, "Spaces of net functions," Mat. Sb., v. 86(128), 1971.

3. L. S. Frank, "Algèbre des opérateurs aux differences finies," Israel J. Math., v. 13, 1972, pp. $24-55$.

4. I. GOHBERG \& L. RODMAN, "On spectral analysis of non-monic matrix and operator polynomials, I," Israel J. Math., v. 30, 1978, pp. 133-151.

5. B. GustafsSon, H.-O. KREISS \& A. SUnDSTRÖM, "Stability theory of difference approximations for mixed initial boundary value problems. II," Math. Comp., v. 26, 1972, pp. 649-686.

6. B. Gustafsson, "The convergence rate for difference approximations to mixed initial boundary value problems," Math. Comp., v. 29, 1975, pp. 396-406.

7. H.-O. Kreiss, "Initial boundary value problems for hyperbolic systems," Comm. Pure Appl. Math., v. 23,1970 , pp. $277-298$.

8. H.-O. KREISS, "Stability theory for difference approximations of mixed initial boundary value problems. I," Math. Comp., v. 22, 1968, pp. 703-714.

9. H.-O. KREISS, "On difference approximations of the dissipative type for hyperbolic differential equations," Comm. Pure Appl. Math., v. 17, 1964, pp. 335-353.

10. H.-O. KREISS, Difference Approximations for Time Dependent Problems, AGARD Lecture Series No. 73, 1975.

11. A. MAJDA \& S. OSHER, "Initial-boundary value problems for hyperbolic equations with uniformly characteristic boundary," Comm. Pure Appl. Math., v. 28, 1975, pp. 607-675.

12. A. Majda, J. McDonough \& S. Osher, "The Fourier method for nonsmooth initial data," Math. Comp., v. 32, 1978, pp. 1041-1081.

13. D. Michelson, Initial-Boundary Value Problems for Hyperbolic Equations and Their Difference Approximation With Uniformly Characteristic Boundary, Ph.D. thesis, Dept. of Math. Sci., Tel-Aviv Univ., June 1980.

14. S. OSHER, "Stability of difference approximations of dissipative type for mixed initial-boundary value problems. I," Math. Comp., v. 23, 1969, pp. 335-340.

15. B. N. PARlett, "Accuracy and dissipation in difference schemes," Comm. Pure Appl. Math., v. 19, 1966, pp. 111-123.

16. J. RaUCH, " $L_{2}$ is a continuable initial condition for Kreiss' mixed problems," Comm. Pure Appl. Math., v. 25, 1972, pp. 265-285.

17. L. SARASON, "Hyperbolic and other symmetrizable systems in regions with corners and edges," Indiana Univ. Math. J., v. 26, No. 1, 1977, pp. 1-39. 